2018-04-01

\title{
Effect of Gyroscope Parameters on Gyroscopic Tremor Suppression in a Single Degree of Freedom
}

Brendon Connor Allen

Brigham Young University

Follow this and additional works at: https://scholarsarchive.byu.edu/etd

Part of the Mechanical Engineering Commons

\section{BYU ScholarsArchive Citation}

Allen, Brendon Connor, "Effect of Gyroscope Parameters on Gyroscopic Tremor Suppression in a Single Degree of Freedom" (2018). All Theses and Dissertations. 6782.

https://scholarsarchive.byu.edu/etd/6782

This Thesis is brought to you for free and open access by BYU ScholarsArchive. It has been accepted for inclusion in All Theses and Dissertations by an authorized administrator of BYU ScholarsArchive. For more information, please contact scholarsarchive@byu.edu, ellen_amatangelo@byu.edu. 
Effect of Gyroscope Parameters on Gyroscopic Tremor

Suppression in a Single Degree of Freedom

\author{
Brendon Connor Allen
}

\begin{abstract}
A thesis submitted to the faculty of
Brigham Young University

in partial fulfillment of the requirements for the degree of

Master of Science
\end{abstract}

Steven K. Charles, Chair

Mark B. Colton

Marc D. Killpack

Department of Mechanical Engineering

Brigham Young University

Copyright (C) 2018 Brendon Connor Allen

All Rights Reserved 


\author{
ABSTRACT \\ Effect of Gyroscope Parameters on Gyroscopic Tremor \\ Suppression in a Single Degree of Freedom \\ Brendon Connor Allen \\ Department of Mechanical Engineering, BYU \\ Master of Science
}

\begin{abstract}
Although tremor is one of the most common movement disorders, there are few effective tremor-suppressing options available to patients. One potential tremor-suppression device involves a wearable gyrostabilizer similar to those used to stabilize cameras. However, we do not currently know how to design a gyrostabilizer to suppress tremor in an optimal manner. To address this gap, we present a systematic investigation of how gyrostabilizer parameters affect tremor suppression in a single degree of freedom (DOF). A simple model of the hand with a single DOF at the wrist and a gyroscope mounted on the back of the hand was used to focus on the most basic effects. After demonstrating that a linearized version of the non-linear equations of motion provides an adequate approximation, we simulated the frequency response of the system (hand + gyroscope) to a tremorogenic input torque at the wrist. By varying system parameters one at a time, we determined the effect of individual parameters on the frequency response of the system. To minimize the bandwidth without adding significant inertia about the wrist joint, the inertia and spin speed of the flywheel should be as high as design constraints allow, whereas the distance from the wrist joint axis to the gyroscope, the precession stiffness, and the precession damping should be kept as low as possible. The results demonstrate the potential of gyroscopic tremor suppression and can serve as the foundation for further investigations of gyroscopic tremor suppression in the upper limb.
\end{abstract}

Keywords: tremor, suppression, gyroscope, gyrostabilizer, wrist, hand 


\section{ACKNOWLEDGEMENTS}

I would like to thank Dr. Steven Charles for his support, effort, and guidance during this project. He greatly assisted me in both the technical aspects of my research as well as in writing my paper and thesis. It was a pleasure working with him. I would also like to thank Dr. Mark Colton and Dr. Marc Killpack for their support and willingness to serve on my thesis committee. I would like to express my thanks to the BYU department of graduate studies for funding my graduate studies. My appreciation extends to those at BYU who have supported my research. This includes my fellow peers in the lab, such as Michael Eyler, who assisted in generating my figures. Most significantly I would like to thank my lovely wife, Breanna, for her constant support and assistance throughout our graduate school experience. 


\section{TABLE OF CONTENTS}

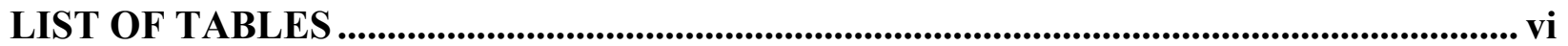

LIST OF FIGURES ...................................................................................................................... vii

NOMENCLATURE.........................................................................................................................

1 Introduction ..................................................................................................................... 1

2 Methods …................................................................................................................................... 3

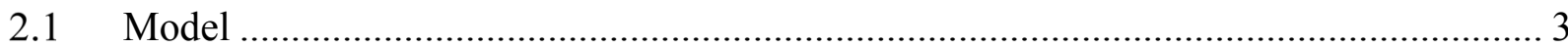

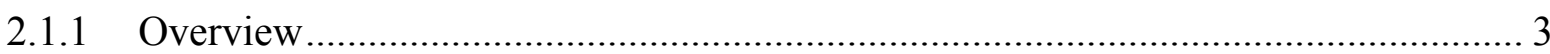

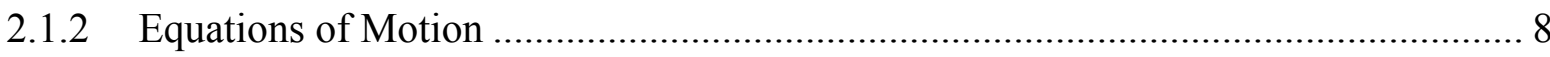

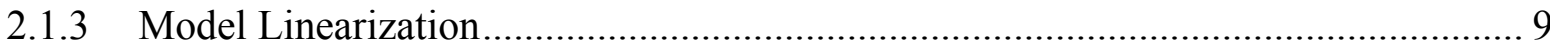

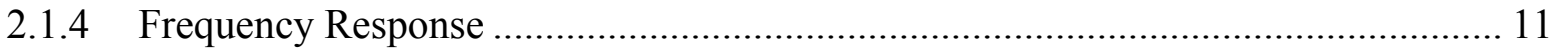

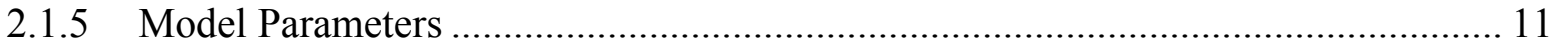

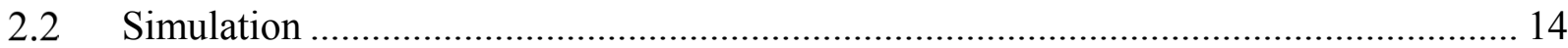

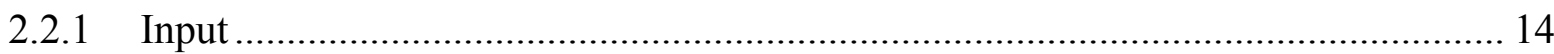

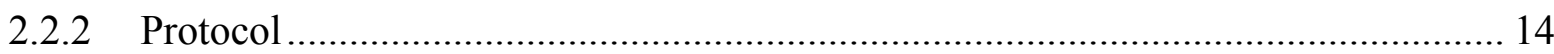

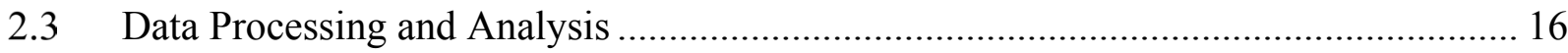

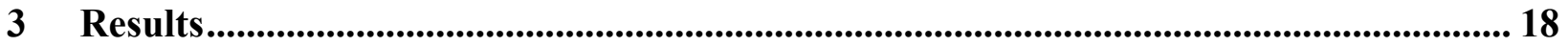

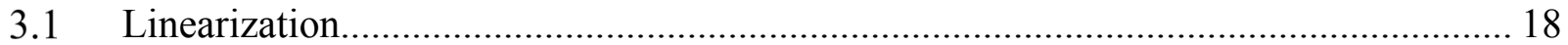

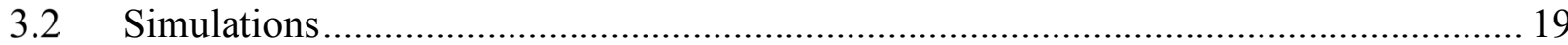

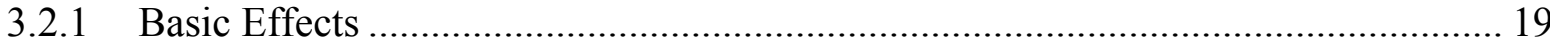

3.2.2 Effect of Individual Parameters on Basic Effect.................................................... 21

3.2.3 Robustness to variations in wrist-hand parameters and gravity ............................... 25

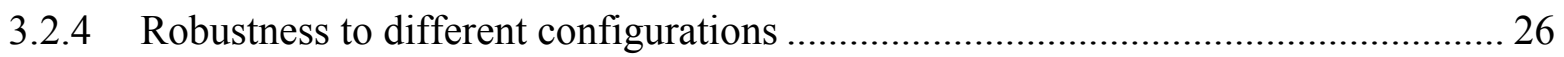

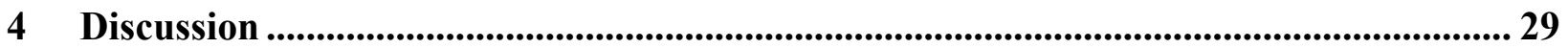

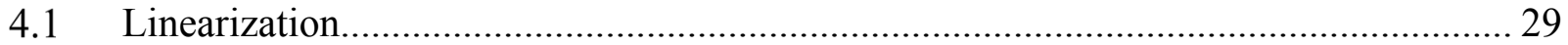

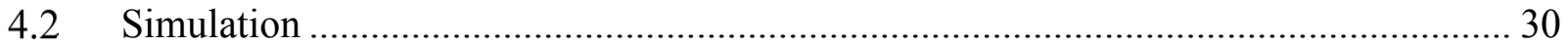

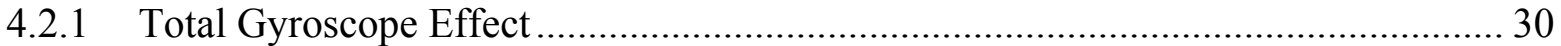

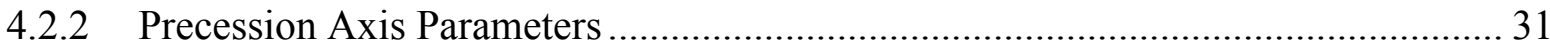

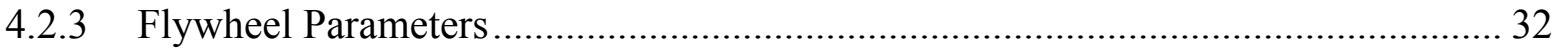

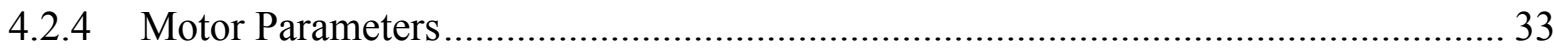

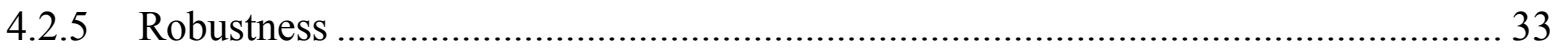




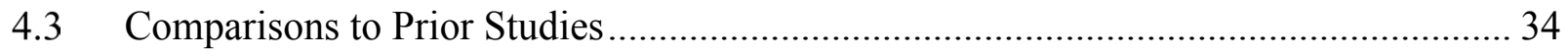

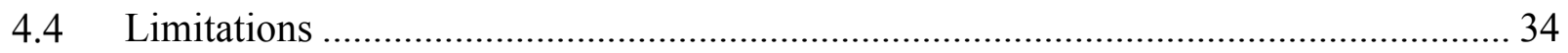

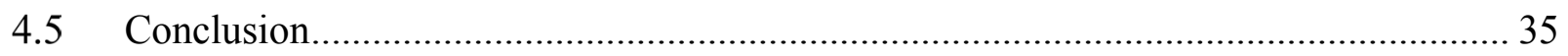

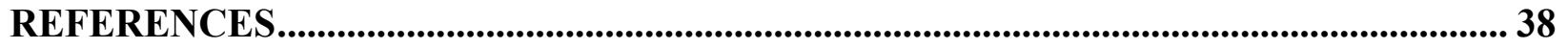

Appendix A. Literature Review …......................................................................................... 44

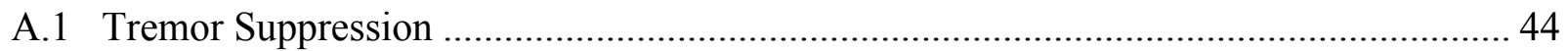

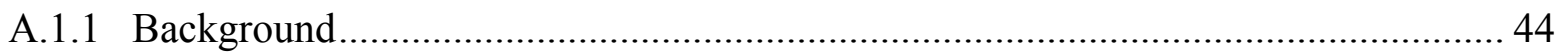

A.1.2 Alternative Methods for Tremor Suppression .......................................................... 44

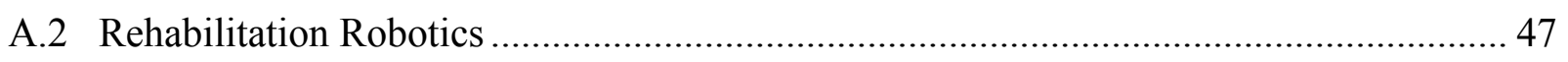

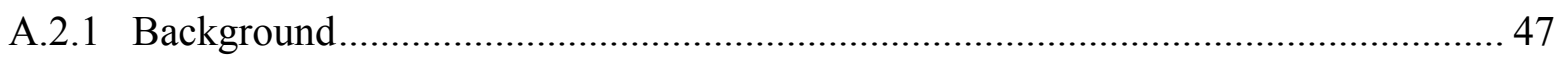

A.2.2 Grand Challenges: How to Optimize Use-Dependent Plasticity ............................... 49

A.2.3 Types of Assistance, Mechanical Designs, and Actuation ........................................ 50

A.2.4 Methods to Induce Plasticity and to Improve a Patient's Health................................ 51

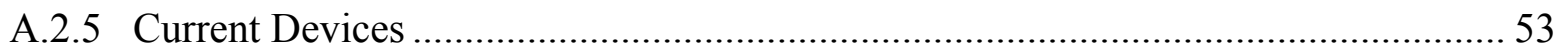

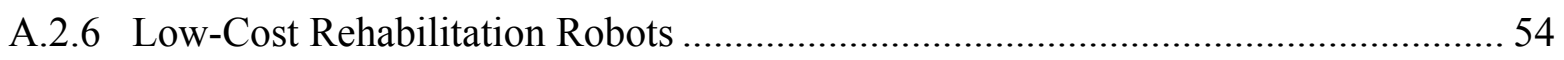

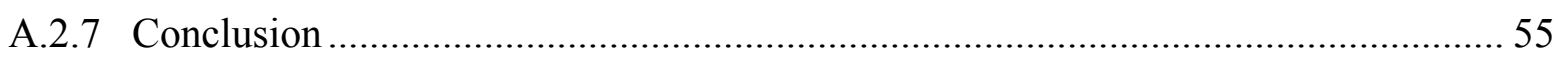

Appendix B. Derivation of State Space Equations of Motion ...............................................5 57

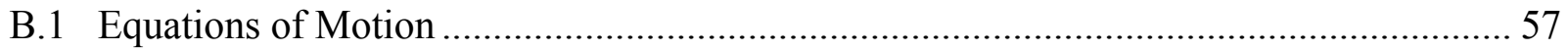

B.1.1 Coordinate Frames and Kinematic Relationships ................................................. 57

B.1.2 Lagrangian for Each Rigid Body ……………….................................................. 59

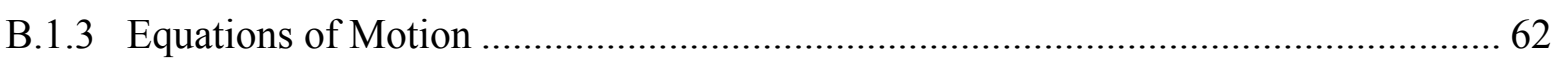

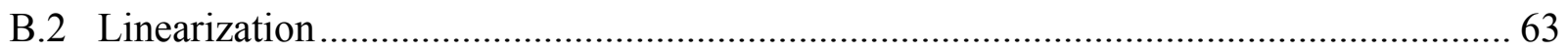

Appendix C. Code to Generate State Space Matrices................................................................. 66

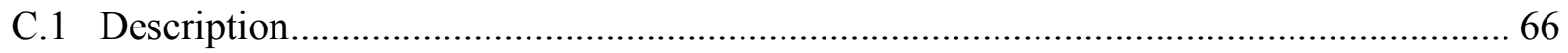

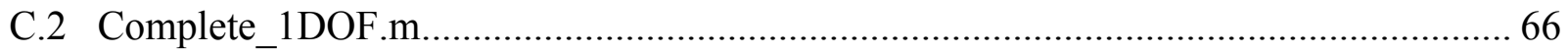

Appendix D. Code for Sensitivity Analysis ……..................................................................... 79

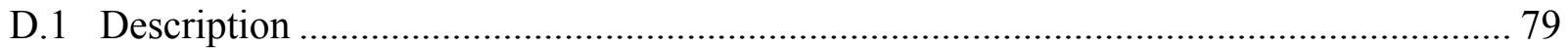

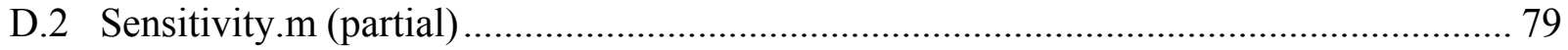

Appendix E. Code to Compare Linear and Nonlinear Systems .......................................... 89

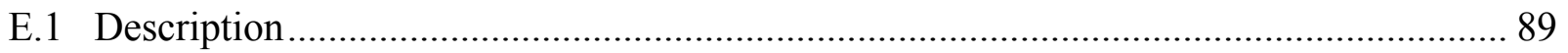

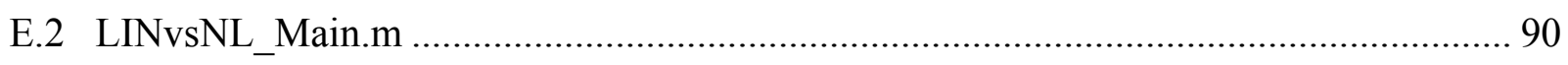

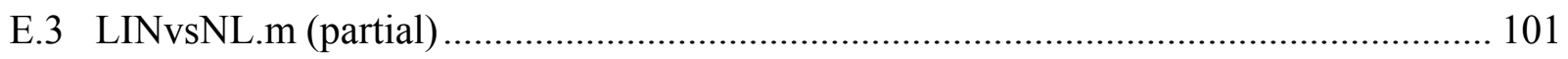




\section{LIST OF TABLES}

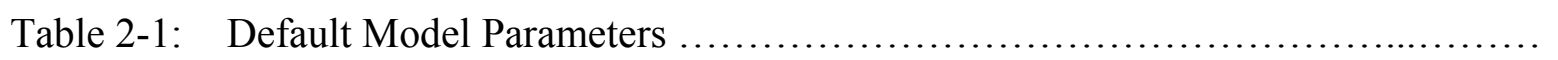

Table 3-1: This table summarizes the difference in frequency response (input=wrist torque, output=wrist angle) between "hand without gyroscope" and "hand with gyroscope-in-off-state" (mass effect), "hand with gyroscope-in-off-state" and "hand with gyroscope-in-on-state" (spin effect), and "hand without gyroscope" and "hand with gyroscope-in-on-state" (Total effect). The symbols --, $, \uparrow, \downarrow$, $\sim \uparrow$, and $\sim \downarrow$ mean there was no effect, negligible effect, increased effect, decreased effect, slight increase, and slight decrease, respectively. A comma means that the results change, for example, $\sim, \uparrow$ means the response is negligible and then it increases.

Table B-1: Rotation Matrix and Angular Velocity of Frame $\boldsymbol{x}_{1} \boldsymbol{y}_{1} \boldsymbol{z}_{1}$ for Each Wrist Joint Axis

Table B-2: The Orientation Constants to Obtain the 6 Cardinal Orientations of a Gyroscope Relative to the Hand

Table B-3: Equilibrium Torques for each Gyroscope Orientation 


\section{LIST OF FIGURES}

Figure 2-1: Gyrostabilizer mounted on back of hand; in this configuration, the wrist joint axis is oriented to allow wrist flexion-extension, and the gyroscope is oriented according to the default orientation (O2). A: View showing the three relevant axes: the wrist joint axis, precession axis, and spin axis. The precession axis $(\boldsymbol{Z}=\boldsymbol{Z})$ is shared by the reference frame of the gimbal structure $(\boldsymbol{X Y Z})$ and the reference frame of the motor and flywheel $(\boldsymbol{x y z})$. The spin axis is the $\boldsymbol{y}$-axis of the $\boldsymbol{x y z}$ frame. B: Side view defining the input torque $T_{w}^{\text {pert }}$, wrist joint angle $\theta$, and precession angle $\phi$, as well as various parameters. Also shown are the inertial reference frame $\left(\boldsymbol{X}_{0} \boldsymbol{Y}_{0} \boldsymbol{Z}_{0}\right)$ and the hand body-fixed frame $\left(\boldsymbol{x}_{1} \boldsymbol{y}_{1} \boldsymbol{z}_{1}\right) \ldots \ldots$

Figure 2-2: With the wrist joint axis representing wrist flexion-extension (as in Figure 2-1), the precession axis $(\boldsymbol{Z}=\boldsymbol{z}$, show in red) and spin axis ( $\boldsymbol{y}$, shown in blue) can take on six different cardinal configurations (ignoring the trivial configuration in which the precession and spin axes are parallel). The flywheel is represented in green, and the wrist joint axis is represented by the black dot. The hand and gyroscope system are in their equilibrium state. ............................

Figure 2-3: Cardinal configurations. If the wrist joint axis is oriented to allow either wrist flexion-extension (flex-ext), wrist radial-ulnar deviation (rad-uln dev), or forearm pronation supination (pro-sup), the three axes (wrist joint axis, precession axis, and spin axis) can be oriented in 18 different configurations (excluding trivial configurations in which the precession and spin axes are parallel), though configurations where the wrist joint and precession axes are parallel (dark gray background) have no spin effect. The remaining 12 configurations are the cardinal configurations. Only configurations in which all three axes are mutually perpendicular (white background) have any practical gyrostabilizing effect on the hand. Configurations where the wrist joint and spin axes are parallel have a negligible spin effect (light gray background). ...........

Figure 3-1: Response vs. time, showing the difference between the response of the nonlinear model (thick solid) and the response of the linearized model (thick dashed) to a sinusoidal input torque (at $8 \mathrm{~Hz}$ ) when the gyroscope is in the onstate (black) or the off-state (gray). For comparison, the response of the hand by itself (without a gyroscope) is provided in thin solid black. The plot includes the initial, transient phase as well as the beginning of the steady-state phase. Note that the linearized model approximates the non-linear model quite well. This simulation was created using the default orientation and parameter values. ......

Figure 3-2: Percent error in magnitude ratio between the nonlinear and linearized models as a function of the steady-state amplitude of wrist displacement $(\theta)$, evaluated at input frequencies of 4,8 , and $12 \mathrm{~Hz}$. To validate the linearization over the entire parameter space the magnitude ratio was calculated for different parameter sets. 
One parameter was varied at a time and set to its default value (thick solid line), its minimum value (dotted line) and then its maximum value (dashed line)......

Figure 3-3: Frequency response of the default system (default orientation and parameter values) with the gyroscope in the on-state (thick solid) and off-state (thick dashed), compared to the response of the hand without gyroscope (thin solid). Adding the gyroscope (in the off-state) to the hand produces the usual low-pass filtering effect associated with increased mass, decreasing the peak frequency and bandwidth. Turning the gyroscope on serves to intensify this low-pass filtering effect, further decreasing the peak frequency and bandwidth of the system. ...................................................................

Figure 3-4: Effect of varying precession axis parameters (Table 2-1) on the frequency response of the default system in the on-state (thick solid) and off-state (thick dashed). Also included for reference is the response of the hand without the gyroscope (thin solid). Subplots depict the influence of the distance from the wrist joint axis to the gyroscope $\left(x_{p}, y_{p}\right.$, and $z_{p}$ depicted in subplots $\mathrm{A}, \mathrm{C}$, and E, respectively) as well as the precession axis stiffness $K_{p}$ and damping $D_{p}$ (subplots B and D). In the default configuration (Figure 2-1), changing $z_{p}$ merely moves the gyroscope parallel to the wrist joint axis and therefore has no effect, as seen in subplot E. The frequency response of the hand by itself (without gyroscope) is the same in each plot because the precession axis parameters have no effect on the hand. Similarly, the frequency response of the gyroscope in the off-state is the same in B and D because the precession stiffness and damping has no effect on the gyroscope in the off-state......................

Figure 3-5: Varying the motor parameters (Table 2-1) has a negligible effect on the frequency response of the default system in the on-state (thick solid) and offstate (thick dashed). For reference, the response of the hand without the gyroscope is also included (thin solid). Subplots depict the influence of the offset of the motor $r_{m}$ from the precession axis to the center of mass of the motor (A), the radius $R_{m}$ and length $L_{m}$ of the cylindrically shaped motor (B and C, respectively), and the density $\rho_{m}$ of the motor (D). The frequency response of the hand is the same in each subplot because varying the motor parameters has no effect on the hand.

Figure 3-6: Effect of varying flywheel parameters (Table 2-1) on the frequency response of the default system in the on-state (thick solid) and off-state (thick dashed), compared to the response of the hand without the gyroscope (thin solid). Subplots depict the influence of the offset of the flywheel $r_{f}$ from the precession axis to the center of mass of the flywheel (A), the radius $R_{f}$ and length $L_{f}$ of the cylindrically shaped flywheel (B and C), and the density $\rho_{f}$ and spin speed $\Omega$ of the flywheel (D and $\mathrm{E}$ ). The frequency response of the hand is the same in each subplot because varying flywheel parameters has zero effect on the hand. 
Figure 4-1 Frequency response of the default configurations using the default parameters with the gyroscope in the on-state (solid blue) and off-state (solid red), versus the frequency response using more optimal parameters with the gyroscope in the on-state (dashed blue) and off-state (dashed red), compared to the response of the hand without the gyroscope (black). Adding the gyroscope in the off-state had a much smaller effect when the optimal parameters were used, however the optimal parameters led to a slightly increased effect when the gyroscope is in

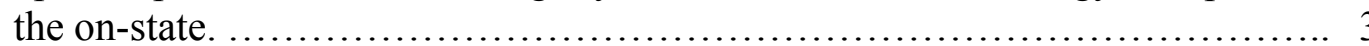




\section{NOMENCLATURE}

\section{General}

FE $\quad$ Flexion-extension

RUD Radial-ulnar deviation

PS Pronation-supination

COM Center of mass

EOM Equation of motion

SS State-space

LTI Linear time invariant

Model Coordinate Frames

$\boldsymbol{X}_{0} \boldsymbol{Y}_{0} \boldsymbol{Z}_{0} \quad$ Inertial reference frame. Origin at the center of the wrist-joint axis.

$\boldsymbol{x}_{1} \boldsymbol{y}_{1} \boldsymbol{z}_{1} \quad$ Hand body-fixed frame. Origin at the center of the wrist-joint axis.

$\boldsymbol{X Y Z}$ Gimbal structure body-fixed frame. Origin at the center of the precession axis.

$x y z \quad$ Motor and flywheel body-fixed frame. Origin at the center of the precession axis.

\section{Model Parameters}

$x_{w} \quad$ Location of the COM of the hand along $x_{1}$

$y_{w} \quad$ Location of the COM of the hand along $\boldsymbol{y}_{1}$

$z_{w} \quad$ Location of the COM of the hand along $z_{1}$

$I_{x w} \quad$ Inertia to rotate the forearm in PS about COM

$I_{y w} \quad$ Inertia to rotate the wrist in RUD about COM

$I_{z w} \quad$ Inertia to rotate the wrist in $\mathrm{FE}$ about COM

$I_{w} \quad$ Inertia to rotate the wrist about the selected wrist joint axis

$m_{w} \quad$ Mass of the hand

$K_{w} \quad$ Wrist joint stiffness in FE, RUD, or PS

$D_{w} \quad$ Wrist joint damping in FE, RUD, or PS

$\zeta \quad$ Damping ratio of the wrist joint axis

$x_{p} \quad$ Location to the center of the precession axis along $\boldsymbol{x}_{1}$

$y_{p} \quad$ Location to the center of the precession axis along $\boldsymbol{y}_{1}$

$z_{p} \quad$ Location to the center of the precession axis along $z_{1}$

$K_{p} \quad$ Stiffness of the precession axis

$D_{p} \quad$ Damping of the precession axis

$r_{m} \quad$ Location of the COM of the motor along $\boldsymbol{y}$ 


$\begin{array}{ll}R_{m} & \text { Radius of the motor } \\ L_{m} & \text { Length of the motor } \\ \rho_{m} & \text { Density of the motor } \\ I_{x m} & \text { Inertia to rotate the motor about } \boldsymbol{x} \\ I_{y m} & \text { Inertia to rotate the motor about } \boldsymbol{y} \\ I_{z m} & \text { Inertia to rotate the motor about } \boldsymbol{z} \\ r_{f} & \text { Location of the COM of the flywheel along } \boldsymbol{y} \\ R_{f} & \text { Radius of the flywheel } \\ L_{f} & \text { Length of the flywheel } \\ \rho_{f} & \text { Density of the flywheel } \\ I_{x f} & \text { Inertia to rotate the flywheel about } \boldsymbol{x} \\ I_{y f} & \text { Inertia to rotate the flywheel about } \boldsymbol{y} \\ I_{z f} & \text { Inertia to rotate the flywheel about } \boldsymbol{z} \\ \Omega & \text { Spin speed of the flywheel }\end{array}$

\section{Equations of Motion and Linearization}

$\theta \quad$ Angular displacement about the wrist joint axis

$\phi \quad$ Angular displacement about the precession axis

$T_{w} \quad$ Perturbation torque about the wrist joint axis

$T_{w}^{e q} \quad$ Equilibrium torque about the wrist joint axis

$T_{w}^{\text {pert }} \quad$ Input torque into the linear system

$T_{p} \quad$ Perturbation torque about the precession axis

$T_{p}^{e q} \quad$ Equilibrium torque about the precession axis

$\boldsymbol{x} \quad$ State vector. States were chosen so that $x_{1}=\theta, x_{2}=\emptyset, x_{3}=\dot{\theta}$, and $x_{4}=\dot{\emptyset}$.

$\boldsymbol{x}^{e q} \quad$ Equilibrium state vector

$\boldsymbol{u}^{e q} \quad$ Equilibrium torque vector

$\boldsymbol{y}^{e q} \quad$ Equilibrium output vector

$\boldsymbol{f}$ Time derivative of the state vector

$g \quad$ Desired output of the system

$\boldsymbol{q} \quad$ Vector of the system's degrees of freedom

$\boldsymbol{F}$ or $\boldsymbol{u}$ The input vector into the nonlinear system

$R_{1} \quad$ Rotation matrix. Transforms from $\boldsymbol{X}_{0} \boldsymbol{Y}_{0} \boldsymbol{Z}_{0}$ to $\boldsymbol{x}_{1} \boldsymbol{y}_{1} \boldsymbol{z}_{1}$.

$R_{2} \quad$ Rotation matrix. Transforms from $\boldsymbol{x}_{1} \boldsymbol{y}_{1} \boldsymbol{z}_{1}$ to $\boldsymbol{X Y Z}$.

$R_{3} \quad$ Rotation matrix. Transforms from $\boldsymbol{X Y Z}$ to $\mathbf{x y z}$.

$\boldsymbol{\omega}_{\boldsymbol{x}_{1} \boldsymbol{y}_{1} \boldsymbol{z}_{1}} \quad$ Angular velocity vector expressed in the $\boldsymbol{x}_{1} \boldsymbol{y}_{1} \boldsymbol{z}_{1}$ coordinate frame

$\beta_{1} \quad$ Orientation constant. So are $\beta_{2}$ and $\beta_{3}$. 
$\boldsymbol{r}_{\boldsymbol{i}} \quad$ Position vector of the hand $(w)$, motor $(m)$, or flywheel $(f)$, depending on $i$

$\boldsymbol{\omega}_{i} \quad$ Angular velocity of the hand $(w)$, motor $(m)$, or flywheel $(f)$, depending on $i$

$T_{i} \quad$ Kinetic energy of the hand $(w)$, motor $(m)$, or flywheel $(f)$, depending on $i$

$V_{i} \quad$ Potential energy of the hand $(w)$, motor $(m)$, or flywheel $(f)$, depending on $i$

$L_{i} \quad$ Lagrangian of the hand $(w)$, motor $(m)$, or flywheel $(f)$, depending on $i$

\section{Frequency Response}

$G(s) \quad$ Transfer function in the Laplace domain

$G(j \omega) \quad$ Transfer function in the frequency domain

$j \quad$ Imaginary number, $j=\sqrt{-1}$.

$\omega \quad$ Frequency in radians

\section{Data Processing and Analysis}

PF Frequency of the resonance peak

PA Amplitude of the resonance peak

BW Bandwidth

ME Mass effect

SE Spin effect

GE Gyroscope effect

-- $\quad$ No effect

$\sim \quad$ Negligible effect

$\uparrow \quad$ Increased effect

$\downarrow \quad$ Decreased effect

$\sim \uparrow \quad$ Slightly increased effect

$\sim \downarrow \quad$ Slightly decreased effect

$\sim, \uparrow \quad$ Negligible effect and then an increased effect 


\section{INTRODUCTION}

Tremor is one of the most common movement disorders [1,2]. It is characterized by an involuntary, rhythmic, oscillatory movement [1] associated with essential tremor, dystonia, Parkinson's disease, cerebellar ataxia, traumatic brain injury, stroke, multiple sclerosis, and other disorders [2,3]. Tremor affects $15 \%$ of people between the ages of 50 and 89 years [4], and more than $65 \%$ of people with upper limb tremor present serious difficulties performing activities of daily living (ADLs) such as eating, dressing, writing, etc. [2].

Unfortunately, the current methods to treat tremor-pharmacotherapy [5] and neurosurgical interventions, including deep brain stimulation (DBS) $[6,7]$-are only partially effective [3]. Medication only reduces tremor by $50 \%$, and only in $50 \%$ of patients $[8,9]$, and can have negative side effects [10] and be subject to habituation [11]. Neurosurgical interventions require invasive surgery, are generally reserved for severe drug-resistant tremor $[8,9]$, and can have serious side effects $[12,13]$. Alternative approaches aim at intervening peripherally and include wearable devices that suppress tremor through passive filtering [14-21], active cancelling with an exoskeleton [22, 23], and electrical stimulation [24-29]. Though promising, these alternative approaches are still under research and are not yet available to patients (see Appendix A).

A potential approach that has received little attention is the use of gyroscopes to mechanically suppress tremor, similar to the use of gyroscopes to stabilize cameras. When a torque 
perturbs the spin axis of a gyroscope's spinning wheel, it generates an additional torque orthogonal to the perturbation torque and the spin, which causes precession [30]. This precession generates an additional torque that resists the original perturbation torque, allowing gyroscopes to act in a stabilizing manner. Systems that utilize this phenomenon are called gyrostabilizers. In addition to stabilizing cameras, gyrostabilizers have been used to stabilize ships, cars, bicycles, spacecraft, and robots [30,31]. The gyrostabilizing phenomenon can also be used to suppress tremor in the upper limb; because gyrostabilizers react instantaneously and proportionally to movement and have the potential to stabilize an entire linkage (and not just a joint), they are potentially wellsuited for tremor suppression. Although two patents have been issued for the use of gyroscopes in tremor suppression $[32,33]$, no research publications on this use of gyroscopes were found. Consequently, the optimal number, location, orientation, degrees of freedom, and physical parameters of tremor-suppressing gyroscopes are unknown. A systematic approach is needed to determine how to maximize the gyroscope's ability to suppress tremor while minimizing adverse effects on the user.

The objective of this work is to understand the fundamental principles of gyroscopic tremor suppression to enable the future development of an optimal gyroscopic tremor suppression device. As this is the first systematic investigation of gyroscopic tremor suppression of which we are aware, we deliberately chose a simple model to focus first on the most basic effects. The model includes a hand with a single degree of freedom (DOF) at the wrist as well as a gyroscope mounted on the back of the hand. We used the model to establish fundamental principles that govern how the parameters and orientation of the gyroscope affect tremor suppression. The principles presented here are relevant to all types of tremor (physiological as well as pathological). 


\section{METHODS}

\subsection{Model}

\subsubsection{Overview}

To simulate the effect of the gyroscope on tremor, we developed a simple model consisting of a hand with a gyroscope mounted on the back of the hand (Figure 2-1). The hand was able to rotate about a single rotational DOF represented by an axis passing through the center of the wrist joint. The gyroscope consisted of a gimbal structure with a single precession axis, the gyroscope motor capable of rotating about the precession axis, and the gyroscope flywheel that rotated about the spin axis of the motor. To thoroughly investigate the effect of the gyroscope, we considered many different configurations of the wrist joint axis, precession axis, and spin axis. More specifically, we allowed the wrist joint axis to point in one of three cardinal directions to allow the hand to rotate in either wrist flexion-extension (FE), wrist radial-ulnar deviation (RUD), or forearm pronation-supination (PS) ${ }^{1}$. Likewise, we allowed the precession axis to point in one of three cardinal orientations relative to the wrist axis, and the spin axis to point in one of two cardinal orientations relative to the precession axis (ignoring the trivial case in which the spin axis is parallel to the precession axis), resulting in six cardinal gyroscope orientations (Figure 2-2). Combining

\footnotetext{
${ }^{1}$ Forearm pronation-supination (PS) occurs as the radial bone rotates about the ulnar bone and therefore occurs proximal to the wrist joint. However, since the axis of rotation passes close to the center of the wrist joint, we lumped PS into the wrist joint for simplicity.
} 
these six cardinal gyroscope orientations with the three cardinal wrist axis orientations (FE, RUD, and PS) yielded 18 possible cardinal configurations (Figure 2-3).
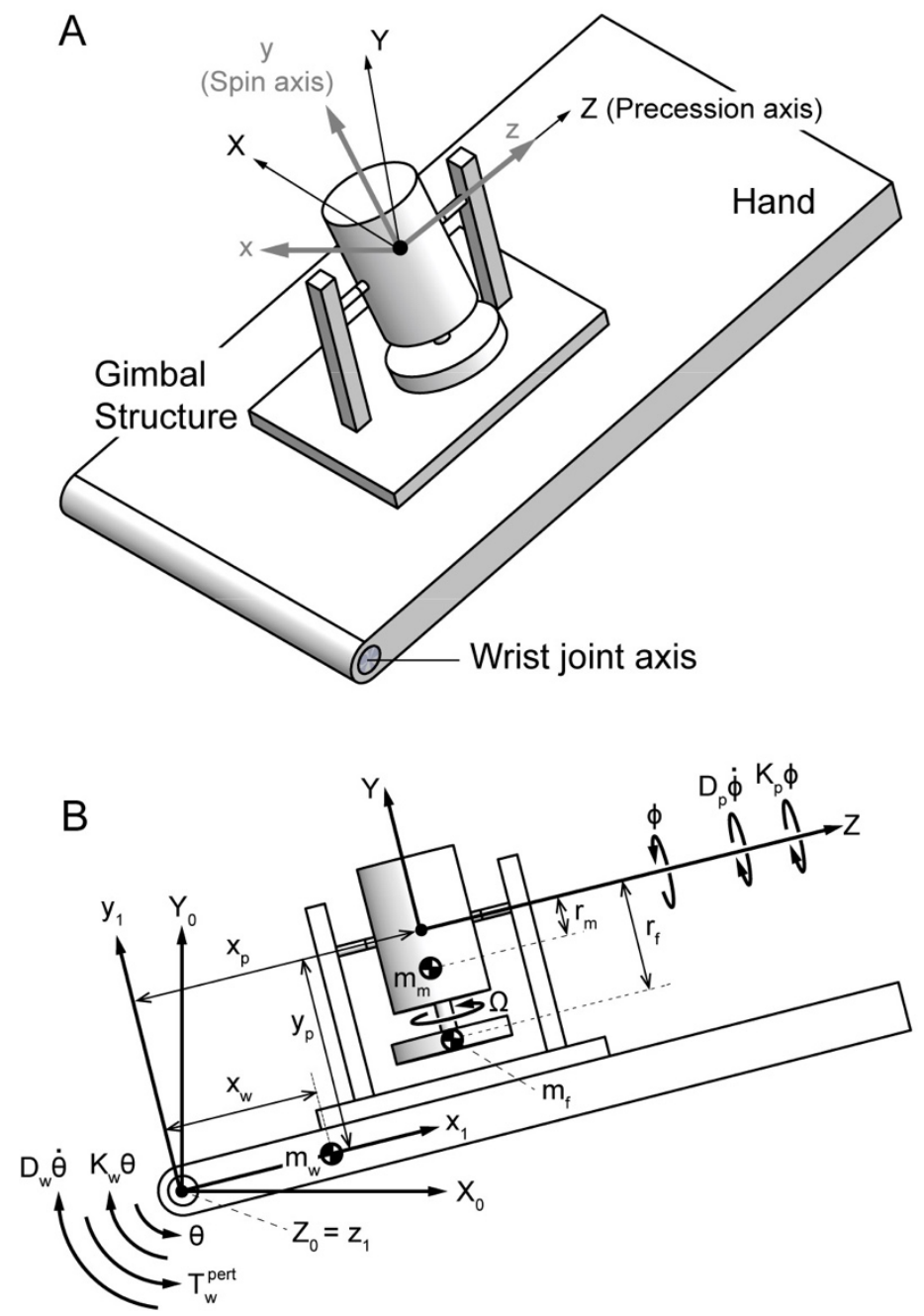

Figure 2-1: Gyrostabilizer mounted on back of hand; in this configuration, the wrist joint axis is oriented to allow wrist flexion-extension, and the gyroscope is oriented according to the default orientation $(\mathrm{O} 2)$. A: View showing the three relevant axes: the wrist joint axis, precession axis, and spin axis. The precession axis $(\boldsymbol{Z}=\mathbf{z})$ is shared by the reference frame of the gimbal structure $(X Y Z)$ and the reference frame of the motor and flywheel $(x y z)$. The spin axis is the $y$-axis of the $x y z$ frame. B: Side view defining the input torque $T_{w}^{p e r t}$, wrist joint angle $\theta$, and precession angle $\phi$, as well as various parameters. Also shown are the inertial reference frame $\left(\boldsymbol{X}_{0} \boldsymbol{Y}_{0} \boldsymbol{Z}_{0}\right)$ and the hand body-fixed frame $\left(\boldsymbol{x}_{1} \boldsymbol{y}_{1} \boldsymbol{z}_{1}\right)$. 

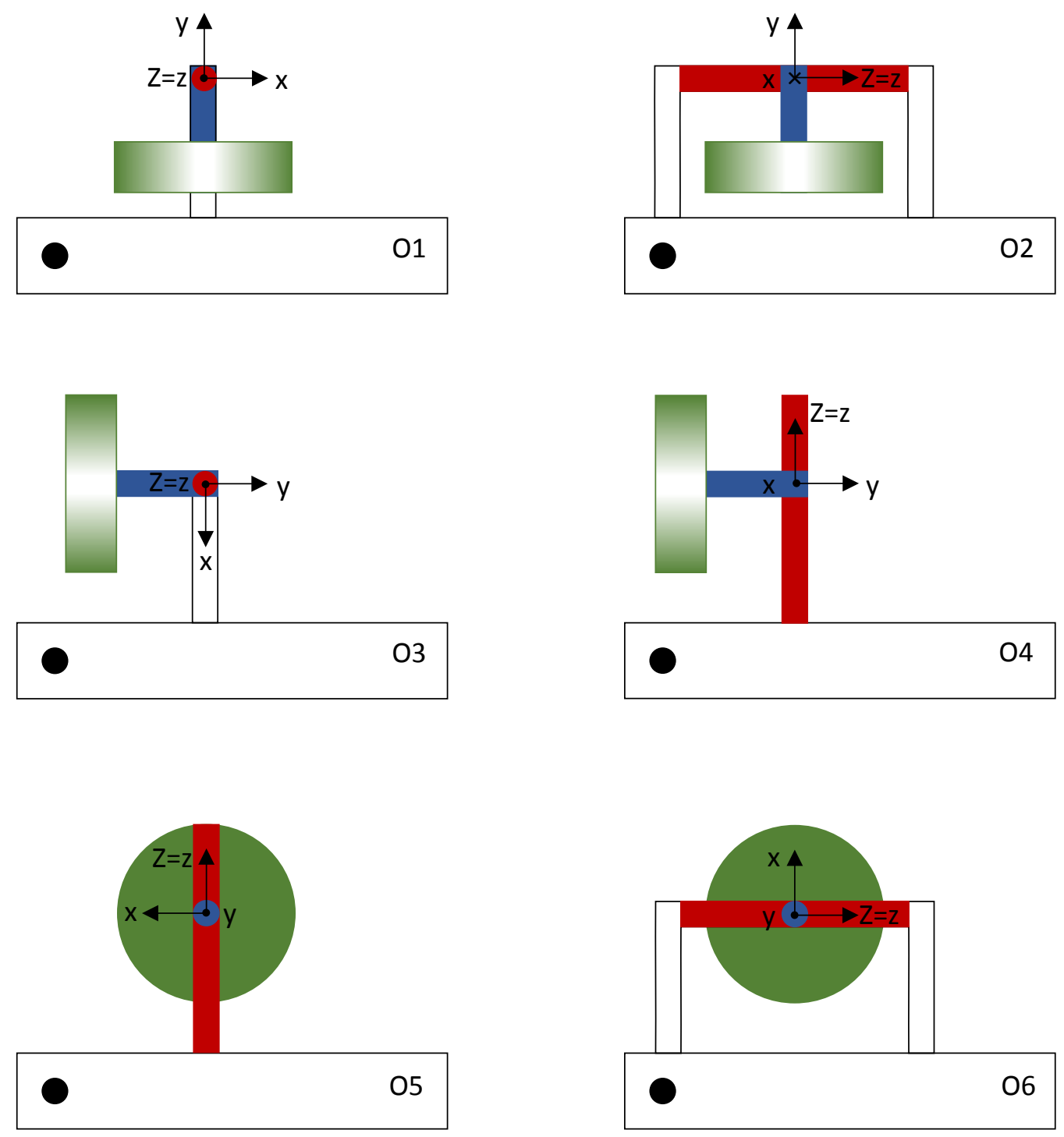

Figure 2-2: With the wrist joint axis representing wrist flexion-extension (as in Figure 2-1), the precession axis $(\boldsymbol{Z}=\boldsymbol{z}$, show in red) and spin axis ( $\boldsymbol{y}$, shown in blue) can take on six different cardinal configurations (ignoring the trivial configuration in which the precession and spin axes are parallel). The flywheel is represented in green, and the wrist joint axis is represented by the black dot. The hand and gyroscope system are in their equilibrium state.

Upon inspection of the 18 cardinal configurations it became clear that 6 will have zero effect. A gyrostabilizer utilizes conservation of angular momentum $(\dot{H}=M)$ to function. If the precession axis and the wrist joint axis are parallel (dark gray background in Figure 2-3) the movement of the wrist will be unable to impart a torque onto the gyroscope and the gyroscope will 


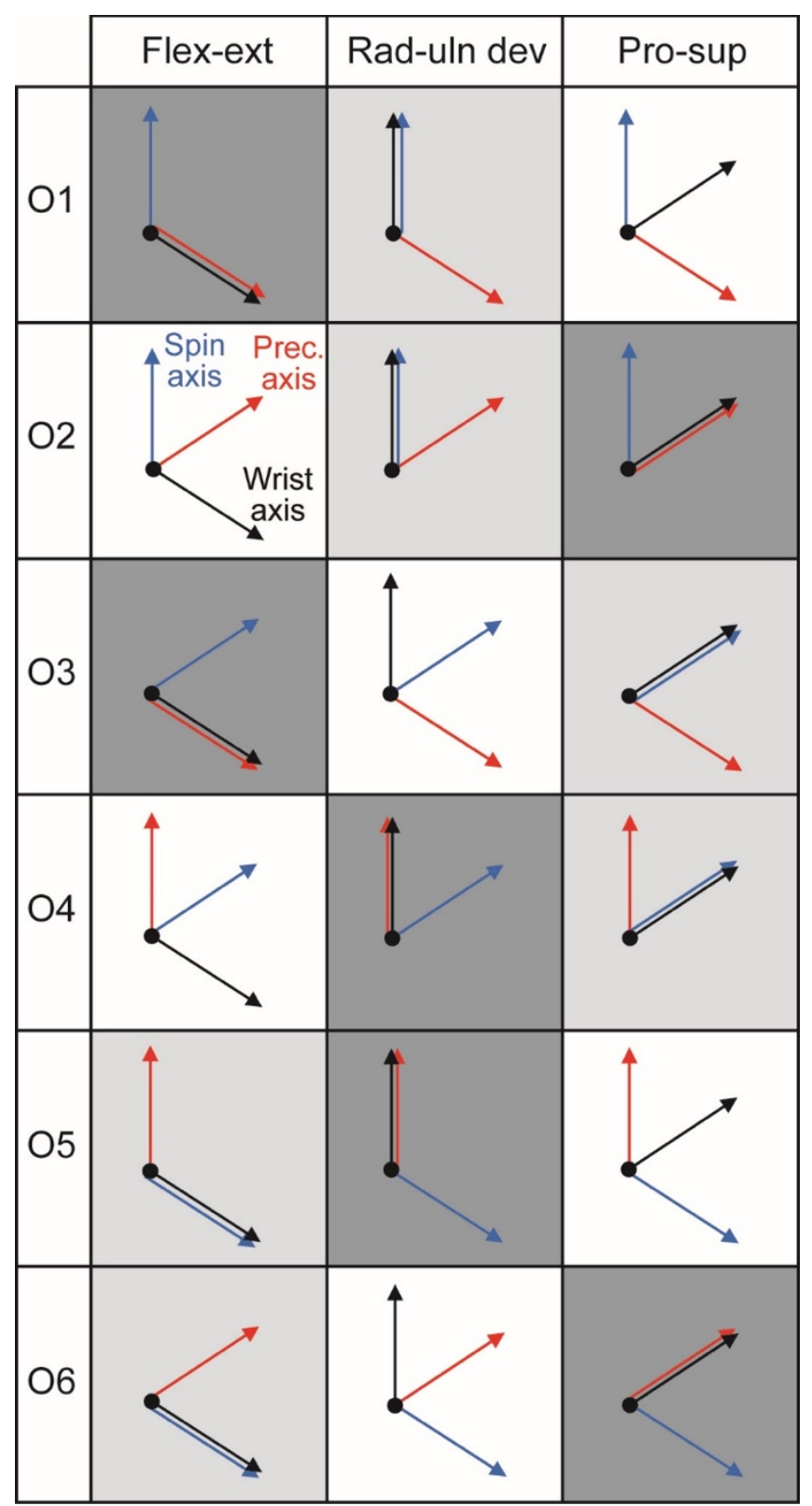

Figure 2-3: Cardinal configurations. If the wrist joint axis is oriented to allow either wrist flexion-extension (flex-ext), wrist radial-ulnar deviation (rad-uln dev), or forearm pronation supination (pro-sup), the three axes (wrist joint axis, precession axis, and spin axis) can be oriented in 18 different configurations (excluding trivial configurations in which the precession and spin axes are parallel), though configurations where the wrist joint and precession axes are parallel (dark gray background) have no spin effect. The remaining 12 configurations are the cardinal configurations. Only configurations in which all three axes are mutually perpendicular (white background) have any practical gyrostabilizing effect on the hand. Configurations where the wrist joint and spin axes are parallel have a negligible spin effect (light gray background).

maintain the same orientation $(\dot{H}=0)$. For the remaining 12 configurations, movement of the wrist causes the angular momentum to change in such a way as to impart a torque about the 
precession axis. This precession then changes the angular momentum again and generates a torque in the direction orthogonal to the precession and spin axes. Therefore, if the spin and the wrist joint axes are initially parallel (light gray background in Figure 2-3) the gyroscope torque is initially orthogonal to the wrist joint axis, but as the gyroscope precesses further the torque will begin to have a component about the wrist joint axis. On the other hand, when the spin, precession, and wrist joint axes are initially mutually orthogonal (white background in Figure 2-3) the initial torque generated by the gyroscope is entirely about the wrist joint axis. The 6 configurations with no effect will not be considered in the remainder of this paper. The remaining 12 configurations will be called the cardinal configurations hereafter.

We simulated the effect of the gyroscope on tremor by inputting a tremorogenic torque (approximated by a sinusoidal torque input) about the wrist joint axis and observing the resulting tremorous displacement about the wrist joint axis and the induced displacement of the gyroscope about the precession axis. To determine the effect of specific gyroscope parameters, we systematically varied gyroscope parameters one at a time and observed the changes in the displacement of the hand about the wrist joint axis (the displacement of the gyroscope about the precession axis was considered more of a by-product).

We have simulated the effect of the gyroscope on tremor for the 12 cardinal combinations. However, for clarity, we present in detail the model and results for one of the cardinal configurations (the "default configuration" shown in Figure 2-1), and we discuss how the results of the other 11 configurations differed from those of the default configuration. The generic model (for any configuration) is given in Appendix B. 


\subsubsection{Equations of Motion}

The equations of motion, which were derived via Lagrange's Method (Appendix B), relate the two input variables (perturbation torques $T_{w}$ and $T_{p}$ about the wrist joint and precession axes, respectively) to the two output variables (angular displacements $\theta$ and $\varnothing$ about the wrist joint and precession axes, respectively):

$$
H \ddot{\boldsymbol{q}}+\boldsymbol{C}=\boldsymbol{F}
$$

where $\boldsymbol{q}=\left[\begin{array}{ll}\theta & \emptyset\end{array}\right]^{T}$ and $\boldsymbol{F}=\left[\begin{array}{ll}T_{w} & T_{p}\end{array}\right]^{T}=\left[\begin{array}{ll}T_{w}^{e q}+T_{w}^{p e r t} & T_{p}^{e q}\end{array}\right]^{T}$, where $T_{w}^{e q}$ and $T_{p}^{e q}$ are the equilibrium torques (Table B-3) about the wrist joint and precession axes, respectively, and $T_{w}^{p e r t}$ is the input into the linear system. The general equations for $H$ and $\boldsymbol{C}$ can be derived following the method described in Appendix B and are too long to present here. However, for the simplified case in which the motor is transparent (motor parameters set to zero) and $y_{w}, z_{w}$, and $z_{p}$ are set to zero, the equations for the default configuration (Figure 2-1) are:

$$
\begin{aligned}
& H=\left[\begin{array}{cc}
m_{f} x_{p}^{2}+m_{f} y_{p}^{2}+m_{f} r_{f}^{2} \cos ^{2} \emptyset+2 m_{f} y_{p} r_{f} \cos \emptyset & -m_{f} x_{p} r_{f} \sin \emptyset \\
+I_{x f} \cos ^{2} \emptyset+I_{y f} \sin ^{2} \emptyset+m_{w} x_{w}^{2}+I_{z w} & \\
-m_{f} x_{p} r_{f} \sin \emptyset & m_{f} r_{f}^{2}+I_{z f}
\end{array}\right] \\
& \boldsymbol{C}=\left[\begin{array}{l}
-2 m_{f} \dot{\theta} \dot{\emptyset} r_{f}^{2} \cos \emptyset \sin \emptyset-2 m_{f} y_{p} r_{f} \dot{\theta} \dot{\emptyset} \sin \emptyset-2 I_{x f} \dot{\theta} \dot{\emptyset} \cos \emptyset \sin \emptyset+K_{w} \theta \\
+I_{y f} \Omega \dot{\varnothing} \cos \emptyset+m_{f} g x_{p} \cos \theta-m_{f} g y_{p} \sin \theta+m_{w} g x_{w} \cos \theta+D_{w} \dot{\theta} \\
-m_{f} g r_{f} \cos \emptyset \sin \theta-m_{f} x_{p} r_{f} \dot{\emptyset}^{2} \cos \emptyset+2 I_{y f} \dot{\theta} \dot{\emptyset} \sin \emptyset \cos \emptyset \\
m_{f} \dot{\theta}^{2} r_{f}^{2} \cos \emptyset \sin \emptyset-I_{y f} \dot{\theta}^{2} \sin \emptyset \cos \emptyset+I_{x f} \dot{\theta}^{2} \cos \emptyset \sin \emptyset+K_{p} \emptyset+D_{p} \dot{\emptyset} \\
+m_{f} y_{p} r_{f} \dot{\theta}^{2} \sin \emptyset-I_{y f} \Omega \dot{\theta} \cos \emptyset-m_{f} g r_{f} \sin \emptyset+D_{p} \dot{\emptyset}
\end{array}\right]
\end{aligned}
$$

Parameter $m_{w}$ represents the mass of the hand; $x_{w}, y_{w}$, and $z_{w}$ locate the center of mass of the hand; $x_{p}, y_{p}$, and $z_{p}$ locate the pivot point of the gyroscope; $r_{f}$ and $r_{m}$ give the location of the center of mass of the gyroscope flywheel and motor, respectively, from the pivot point; and $m_{f}$ and $m_{m}$ are the mass of the gyroscope flywheel and motor, respectively. $K_{w}$ and $D_{w}$ represent the 
joint stiffness and damping about the wrist axis, and $K_{p}$ and $D_{p}$ represent the rotational stiffness and damping about the precession axis (Figure 2-1). The rotational stiffness was necessary to limit the precession angle to a range that is plausible for a design in which the gyroscope is mounted on the back of the hand (without this stiffness the precession angle could increase indefinitely). The rotational damping represents friction between the precession axis and the gyroscope.

\subsubsection{Model Linearization}

Postural tremor consists of relatively small displacements about an equilibrium point and can therefore be approximated by a linear model, which would allow the use of principles and tools from linear systems theory. We performed a local linearization about an equilibrium point to obtain the linear time-invariant (LTI) state-space (SS) form of the system (see Appendix B for details). States were chosen to be $x_{1}=\theta$ (displacement angle of hand), $x_{2}=\varnothing$ (precession angle), $x_{3}=\dot{\theta}$, and $x_{4}=\dot{\emptyset}$. The equilibrium state was chosen as $x^{e q}=\left[\begin{array}{llll}0 & 0 & 0 & 0\end{array}\right]^{\mathrm{T}}$. In other words, the $X_{0}$ and $Z_{0}$ axes formed the horizontal plane, gravity acted parallel to the $Y_{0}$ axis (Figure 2-1), and the hand and gyroscope were at rest. To position the hand and the gyroscope in the desired equilibrium state (Figure 2-2), equilibrium torques were required about the wrist joint and precession axes to offset gravity. These torques were provided by the human at the wrist joint axis and by the precession stiffness at the precession axis. For the default configuration, the equilibrium torques were: $T_{w}^{e q}=g\left(m_{f} x_{p}+m_{m} x_{p}+m_{w} x_{w}\right), T_{p}^{e q}=0$. For the default configuration, gravity does not affect the gyroscope in equilibrium position, so no equilibrium torque was needed about the precession axis. The desired output was chosen to be the displacement angle of the hand $\left(x_{1}\right)$, resulting in the following SS equations: 


$$
\begin{gathered}
\delta \dot{x}=A \delta x+B \delta u \\
\delta y=C \delta x+D \delta u
\end{gathered}
$$

where,

$$
\begin{gathered}
A=\left[\begin{array}{cccc}
0 & 0 & 1 & 0 \\
0 & 0 & 0 & 1 \\
\left(m_{f} g\left(r_{f}+y_{p}\right)+m_{w} g y_{w}\right. & 0 & -D_{w} / C_{1} & -I_{y f} \Omega / C_{1} \\
\left.+m_{m} g\left(r_{m}+y_{p}\right)-K_{w}\right) / C_{1} & & & \\
0 & \left(-K_{p}+\left(m_{f} r_{f}+m_{m} r_{m}\right) g\right) / C_{2} & I_{y f} \Omega / C_{2} & -D_{p} / C_{2}
\end{array}\right] \\
B=\left[\begin{array}{c}
0 \\
0 \\
1 / C_{1} \\
0
\end{array}\right], \quad C=\left[\begin{array}{llll}
1 & 0 & 0 & 0
\end{array}\right], \quad D=\left[\begin{array}{l}
0 \\
0
\end{array}\right]
\end{gathered}
$$

and

$$
\begin{gathered}
C_{1}=I_{x m}+I_{z w}+I_{x f}+m_{f}\left(r_{f}^{2}+x_{p}^{2}+y_{p}^{2}+2 r_{f} y_{p}\right)+m_{w}\left(x_{w}^{2}+y_{w}^{2}\right) \\
+m_{m}\left(r_{m}^{2}+x_{p}^{2}+y_{p}^{2}+2 r_{m} y_{p}\right) \\
C_{2}=I_{z m}+I_{z f}+m_{f} r_{f}^{2}+m_{m} r_{m}^{2}
\end{gathered}
$$

To determine how closely the linear model approximated the non-linear model, we input a sinusoidal input torque, $\delta u=T_{w}^{p e r t}=A * \sin (2 \pi f t)$, into the linear model and we input $\boldsymbol{u}=$ $\left[T_{w}^{e q}+T_{w}^{p e r t} T_{p}^{e q}\right]^{T}$ into the nonlinear model and calculated the steady-state amplitude of the resulting wrist displacements for both the linear $\left(A_{L i n}\right)$ and nonlinear model $\left(A_{N L}\right)$. The magnitude ratio, at input frequency $f$, was then calculated for both the linear $\left(M R_{L i n}=A_{L i n} / A\right)$ and nonlinear $\left(M R_{N L}=A_{N L} / A\right)$ models by dividing the linear and nonlinear steady-state amplitudes of the wrist angle by the input amplitude $(A)$. The percent error was calculated using \%Error = $100\left(M R_{N L}-M R_{L i n}\right) / M R_{N L}$. This process was then repeated for 13 other input amplitudes $(A)$ to determine how the percent error changes as the amplitude of the wrist displacement increases. To determine the effect of the input frequency $(f)$, the above steps were repeated with input 
frequencies of 4, 8, and $12 \mathrm{~Hz}$ to cover the tremor band. Lastly, to determine the effect of the parameters on the linearization, each parameter was changed to its lowest value and then to its highest value found in Table 2-1 (see step 2 of the Protocol section below) and the above process was then repeated again.

\subsubsection{Frequency Response}

As mentioned above, the model has one input (the torque about the wrist axis) and one output of interest (the rotational displacement about the wrist axis); the displacement of the gyroscope about the precession axis was considered a by-product. The relationship between the input and output of interest is given by the transfer function $G(s)=\theta(s) / T(s)$, which we derived from the linearized SS model of the system using the tf function in Matlab. Given the application to tremor suppression, we were particularly interested in the response of the system to tremorogenic input torque. Approximating tremorogenic torque as sinusoidal, the response of the system to tremorogenic input torque is given by the system's frequency response. According to linear system theory [34], the magnitude ratio and phase shift of the system in response to input at frequency $\omega$ can be calculated from the transfer function $G(s)$ as $|G(j \omega)|$ and $\measuredangle G(j \omega)$, where $j=$ $\sqrt{-1}$

\subsubsection{Model Parameters}

To determine the effect of various gyroscope parameters on the frequency response of the system, we first established the frequency response of the system with default parameters; then, we changed the parameters and observed the change in the frequency response. The default parameters are presented here, and the changes in the default parameters are presented below (Simulation Protocol). The default parameter values (Table 2-1, see below for explanation of each 
parameter value) were selected to represent a plausible design for a tremor suppression device mounted on the back of the hand. That said, the exact values are not critical since we systematically investigated the effect of variations around these default values.

Table 2-1: Default Model Parameters

\begin{tabular}{|c|c|c|c|c|c|c|}
\hline \multicolumn{3}{|c|}{ Parameter } & Description & Unit & Default & Variation Values \\
\hline \multirow{9}{*}{\multicolumn{2}{|c|}{ 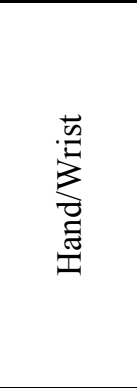 }} & $x_{w}$ & Location of the COM of the hand along $x_{1}$ & $\mathrm{~cm}$ & 6.3 & 5.8 and 6.8 \\
\hline & & $y_{w}$ & Location of the COM of the hand along $\boldsymbol{y}_{1}$ & $\mathrm{~cm}$ & 0 & N/A \\
\hline & & $z_{w}$ & Location of the COM of the hand along $z_{1}$ & $\mathrm{~cm}$ & 0 & N/A \\
\hline & & $I_{x w}$ & Inertia to rotate the forearm in PS about COM & $\mathrm{kg} \cdot \mathrm{m}^{2}$ & 0.0013 & 0.0008 and 0.0018 \\
\hline & & $I_{y w}$ & Inertia to rotate the wrist in RUD about COM & $\mathrm{kg} \cdot \mathrm{m}^{2}$ & 0.00095 & 0.0006 and 0.0013 \\
\hline & & $I_{z w}$ & Inertia to rotate the wrist in FE about COM & $\mathrm{kg} \cdot \mathrm{m}^{2}$ & 0.00065 & 0.0004 and 0.0009 \\
\hline & & $m_{w}$ & Mass of the hand & $\mathrm{kg}$ & 0.40 & 0.35 and 0.45 \\
\hline & & $K_{w}$ & Wrist joint stiffness in FE, RUD, or PS & $\mathrm{Nm} / \mathrm{rad}$ & 1 & 5 and 10 \\
\hline & & $D_{w}$ & Wrist joint damping in FE, RUD, or PS & $\mathrm{Nms} / \mathrm{rad}$ & 0.033 & 0.066 and 0.132 \\
\hline \multirow{14}{*}{ 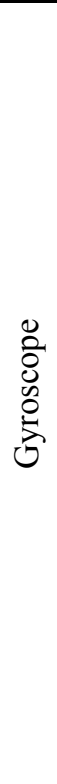 } & \multirow{5}{*}{ 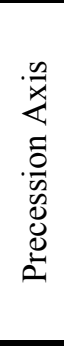 } & $x_{p}$ & $\begin{array}{l}\text { Location to the center of the precession axis } \\
\text { along } \boldsymbol{x}_{1}\end{array}$ & $\mathrm{~cm}$ & 4.0 & 2.0 and 6.0 \\
\hline & & $y_{p}$ & $\begin{array}{l}\text { Location to the center of the precession axis } \\
\text { along } \boldsymbol{y}_{1}\end{array}$ & $\mathrm{~cm}$ & 3.0 & 2.5 and 3.5 \\
\hline & & $z_{p}$ & $\begin{array}{l}\text { Location to the center of the precession axis } \\
\text { along } z_{1}\end{array}$ & $\mathrm{~cm}$ & 0 & -2 and 2 \\
\hline & & $K_{p}$ & Stiffness of the precession axis & $\mathrm{Nm} / \mathrm{rad}$ & 5 & $1,2.5,10$, and 20 \\
\hline & & $D_{p}$ & Damping of the precession axis & $\mathrm{Nms} / \mathrm{rad}$ & 0.1 & $0.05,0.2,1$, and 5 \\
\hline & \multirow{4}{*}{$\stackrel{0}{\tilde{s}^{0}}$} & $r_{m}$ & Location of the COM of the motor along $y$ & $\mathrm{~cm}$ & -1.0 & $-1.5,0.0$, and 1.0 \\
\hline & & $R_{m}$ & Radius of the motor & $\mathrm{cm}$ & 1.0 & 0.5 and 2.0 \\
\hline & & $L_{m}$ & Length of the motor & $\mathrm{cm}$ & 3.0 & 2.0 and 4.0 \\
\hline & & $\rho_{m}$ & Density of the motor & $\mathrm{kg} / \mathrm{m}^{3}$ & 3750 & 1875 and 7500 \\
\hline & \multirow{5}{*}{$\frac{\vec{\Phi}}{\stackrel{D}{3}}$} & $r_{f}$ & Location of the COM of the flywheel along $y$ & $\mathrm{~cm}$ & -1.0 & $-1.5,0.0$, and 1.0 \\
\hline & & $R_{f}$ & Radius of the flywheel & $\mathrm{cm}$ & 3.0 & 2.5 and 3.5 \\
\hline & & $L_{f}$ & Length of the flywheel & $\mathrm{cm}$ & 2.0 & 1.0 and 3.0 \\
\hline & & $\rho_{f}$ & Density of the flywheel & $\mathrm{kg} / \mathrm{m}^{3}$ & 7850 & 2700 and 11340 \\
\hline & & $\Omega$ & Spin speed of the flywheel & rpm & 10,000 & $\begin{array}{l}0,2000,4000,6000 \\
8000, \text { and } 12000\end{array}$ \\
\hline
\end{tabular}

Hand Parameters: Parameters $x_{w}, y_{w}$, and $z_{w}$ located the center of mass (COM) of the hand relative to the wrist joint axis in terms of the $\boldsymbol{x}_{1} \boldsymbol{y}_{1} \boldsymbol{z}_{1}$ body-fixed frame of the hand (Figure 2-1B). Parameters $y_{w}$ and $z_{w}$ were included in the model but set to zero for the simulations, placing the COM of the hand along the $\boldsymbol{x}_{1}$ axis. The default values for $x_{w}$, the mass of the hand $\left(m_{w}\right)$, and the inertia of the hand about its center of mass $\left(I_{x w}, I_{y w}\right.$, or $I_{z w}$, depending on the wrist joint axis) were taken as the average between typical male and female values from the body- 
segment parameter literature [35]. ${ }^{2}$ For simplicity, wrist joint stiffness and damping were assumed to be time-invariant. The default value for wrist joint stiffness $\left(K_{w}\right)$ represents an order-ofmagnitude estimate of passive stiffness (i.e. in the absence of muscle contraction) for FE, RUD, or PS and was taken from past measurements [36-38]. In the simulations, we also tested higher levels of joint stiffness representing active stiffness (i.e. in the presence of muscle contraction) see below. Joint damping tends to increase with muscle activity as well, but not at the same rate as joint stiffness. Perreault et al showed that shoulder-elbow stiffness and damping tended to increase with muscle activity such that the damping ratio, $\zeta=D_{w} /\left(2 \sqrt{K_{w} I_{w}}\right)$, remained relatively constant [39]. Therefore, we calculated joint damping as $D_{w}=2 \zeta \sqrt{K_{w} I_{w}}$, where $I_{w}=I_{z w}+$ $m_{w} x_{w}^{2}$ was the inertia of the hand about the wrist joint. Studies have shown the wrist joint to be underdamped with $\zeta$ around $0.25-0.5[40,41]$, so we chose $\zeta=0.375$.

Gyroscope Parameters: Parameters $x_{p}, y_{p}$, and $z_{p}$ located the center of the precession axis (i.e. pivot point of the gyroscope) in the $\boldsymbol{x}_{1} \boldsymbol{y}_{1} \boldsymbol{z}_{1}$ frame (Figure 2-1B) $x_{p}$ and $z_{p}$ placed the gyroscope near the center of the hand. $y_{p}$ placed the precession axis slightly above the hand, high enough to allow the gyroscope to precess all the way around. $K_{p}$ and $D_{p}$ represented the rotational stiffness and damping of the gyroscope around the precession axis. Little was known about $K_{p}$ and $D_{p}$, so we included a wide range of variation values (see below). The gyroscope was composed of two bodies: the motor and the flywheel. Parameters $r_{f}$ and $r_{g}$ were used to offset the COM of the motor and flywheel from the precession axis. Their default values place the flywheel and motor nearer to the hand than the precession axis without interference. Both the motor and flywheel were

\footnotetext{
${ }^{2}$ Since rotation about the $x$-axis represents forearm pronation-supination, $I_{w x}$ included the inertia of the hand and forearm about the $x$-axis.
} 
modeled as cylinders. We specified the default radius, length, and density of the motor and flywheel, from which we calculated their mass and inertias as $m_{i}=\rho_{i} L_{i} \pi R_{i}^{2}, I_{x i}=I_{y i}=$ $(1 / 4) m_{i} R_{i}^{2}+(1 / 12) m_{i} L_{i}^{2}$ and $I_{z i}=(1 / 2) m_{i} R_{i}^{2}$, where $i$ represents $m$ (motor) or $f$ (flywheel). The default density, radius, and length of the motor were taken as the average values of three small, standard DC motors (DC precision motors by Maxon, Sachseln, Switzerland). The radius and length of the flywheel were selected to fit over the hand. The default density of the flywheel is the density of steel. The speed of the flywheel $(\Omega)$ was given a default value that is reasonable for a standard DC motor.

\subsection{Simulation}

\subsubsection{Input}

To simulate the effect of the gyroscope on tremor, we drove the wrist with a sinusoidal input torque at frequencies between 4 and $12 \mathrm{~Hz}$ (the tremor band), where most tremor frequencies reside [42]. Whereas tremorogenic muscle activity is clearly non-sinusoidal, the resulting joint torque, which is low-pass filtered by the excitation-contraction dynamics of the muscle, is close to sinusoidal. Therefore, we characterized the effect of the gyroscope on tremor using the frequency response of the system throughout the tremor band.

\subsubsection{Protocol}

The goal of the protocol was to determine the effect of varying model parameters and configurations on tremor suppression. This section details the order and manner in which model parameters and configurations were varied. For each variation, new LTI SS matrices were created, from which the resulting frequency response was obtained. 
Step 1. Establish basic effect of gyroscope on tremor: First, we established the basic effect of the gyroscope in the default configuration (Figure 2-1) with the default parameters (Table 2-1). The basic effect can be broken into two parts: the effect caused by the mass of the gyroscope when the gyroscope is turned off and the additional effect caused by the spinning flywheel when the gyroscope is turned on. Therefore, to determine the basic gyroscope effect, we used the default parameters to calculate and compare the frequency responses of the hand by itself, the hand with the gyroscope turned off, and the hand with the gyroscope turned on.

Step 2. Characterize effect of varying gyroscope parameters on basic effect: Second, we tested the effect of varying gyroscopic parameters on the basic gyroscope effect. In each test, one parameter was varied at a time, with all other parameters at their default values. The range in values over which parameters were varied (Table 2-1) were selected to cover a reasonable design space for a gyrostabilizing device mounted on the back of a hand. When possible, variations bracketed the default value. Three ranges warrant particular mention: the flywheel density was varied from the density of aluminum to that of lead, and the precession stiffness and damping were varied through a relatively large range because they are quite unconstrained in a plausible design. To allow for comparison, the frequency responses of all variations of a given parameter were plotted together. For comparison, we added to each plot the frequency responses of the hand by itself and the gyroscope in the off-state.

Step 3. Determine robustness of effects to variations in wrist-hand parameters and gravity: Third, we determined the robustness of the effects characterized in step 2 to changes in wrist and hand parameters. More specifically, we first repeated step 2, but with hand parameters representing either average female values or average male values [35] as opposed to the average of male and female values used as default values (Table 2-1). Next, since the default values of wrist stiffness 
and damping represented the passive muscle state, we repeated step 2 with values of wrist stiffness or damping (one at a time) representing increasing amounts of muscle contraction (Table 2-1) [3638], with the hand parameters at their default values. Lastly, an effective gyrostabilizer needs to be robust against changes in the orientation of the hand relative to gravity. Therefore, we also ascertained the influence of gravity by repeating steps 1-3 without gravity. By repeating this step in configurations parallel to gravity and configurations perpendicular to gravity (step 4 below), we determined the effect of re-orienting the wrist joint axis relative to gravity.

Step 4: Identify robustness of effects to different configurations: Fourth, we determined if the other 11 configurations (Figure 2-3) behaved similarly to the default configuration. More specifically, we repeated steps 1-3 for each of the other 11 configurations.

\subsection{Data Processing and Analysis}

The goal of this paper is to identify general trends in the tremor-suppressing ability of the gyroscope that occur from varying gyroscope parameters and configurations. To quantify these trends, we extracted three measures from the frequency response of each simulation: the frequency of the resonance peak (PF), the amplitude of the resonance peak (PA), and the bandwidth (BW). Since the purpose of a gyrostabilizer is to pass intended movements and filter out unintended movements (such as tremor), we used the BW measure to characterize the frequencies passed by the filter. The BW was defined as the range of frequencies for which the magnitude ratio was greater than or equal to $70.1 \%$ of its DC-value (i.e. its value at zero frequency).

As mentioned above, the effect of the gyroscope on tremor can be broken into two parts: the effect caused by the mass of the gyroscope when the gyroscope is turned off and the additional effect caused by the spinning flywheel when the gyroscope is turned on. Hereafter we will refer to 
the former as the "mass effect", the latter as the "spin effect", and the combination as the "total gyroscope effect". Therefore, we quantified the mass effect, spin effect, and total gyroscope effect in terms of each of the three measures as follows:

$$
\begin{gathered}
\Delta P F_{M E}=P F_{o f f}-P F_{h a n d} \\
\Delta P A_{M E}=P A_{o f f}-P A_{\text {hand }} \\
\Delta B W_{M E}=B W_{o f f}-B W_{\text {hand }} \\
\Delta P F_{S E}=P F_{\text {on }}-P F_{\text {off }} \\
\Delta P A_{S E}=P A_{\text {on }}-P A_{\text {off }} \\
\Delta B W_{S E}=B W_{\text {on }}-B W_{\text {off }} \\
\Delta P F_{G E}=P F_{\text {on }}-P F_{\text {hand }} \\
\Delta P A_{G E}=P A_{\text {on }}-P A_{\text {hand }} \\
\Delta B W_{G E}=B W_{\text {on }}-B W_{\text {hand }}
\end{gathered}
$$

where subscripts $M E, S E$, and $G E$ represent the mass effect, spin effect, and total gyroscope effect, respectively. Subscripts hand, off, and on refer to the hand by itself, the hand with a gyroscope in the off-state, and the hand with a gyroscope in the on-state. We calculated the changes in each effect for all variations listed in the protocol above and used these changes to determine general trends that were robust across parameters and configurations. 


\section{RESULTS}

\subsection{Linearization}
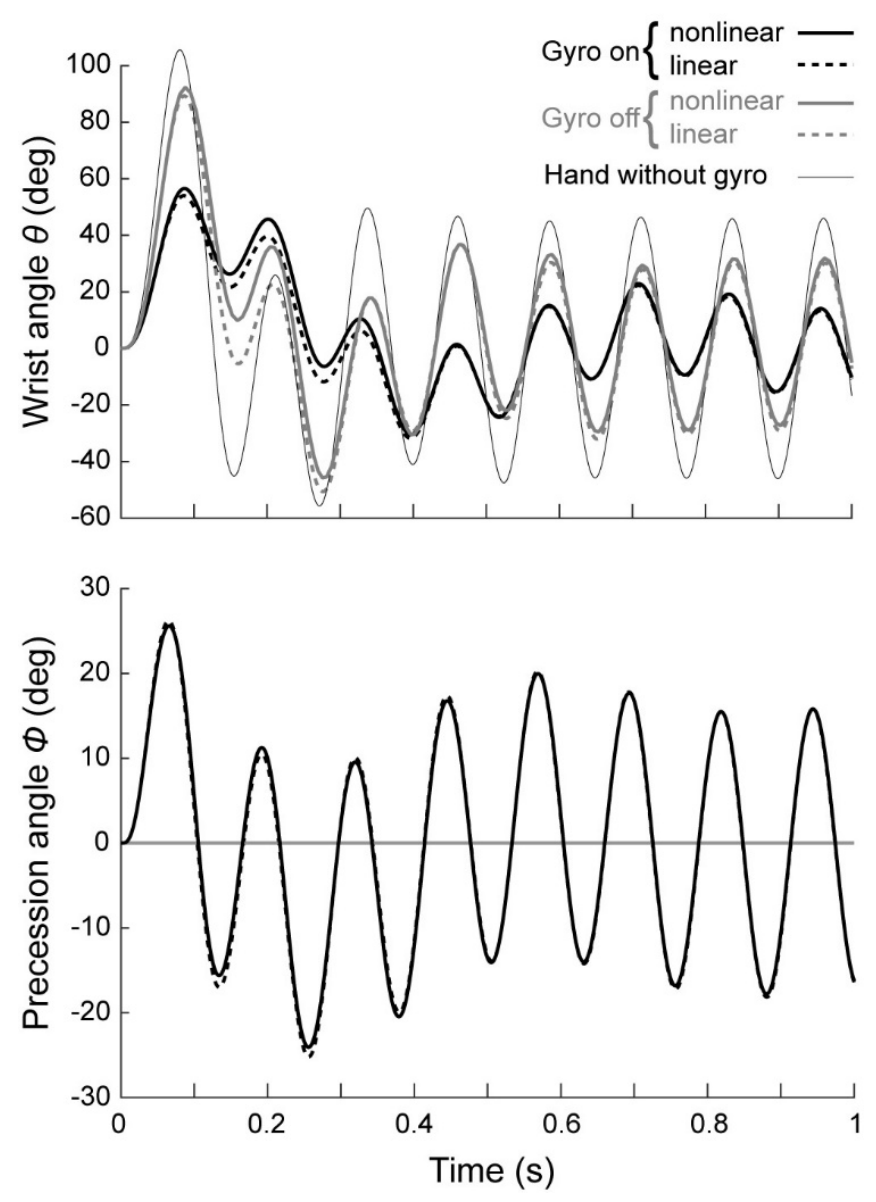

Figure 3-1: Response vs. time, showing the difference between the response of the non-linear model (thick solid) and the response of the linearized model (thick dashed) to a sinusoidal input torque (at $8 \mathrm{~Hz}$ ) when the gyroscope is in the on-state (black) or the off-state (gray). For comparison, the response of the hand by itself (without a gyroscope) is provided in thin solid black. The plot includes the initial, transient phase as well as the beginning of the steady-state phase. Note that the linearized model approximates the non-linear model quite well. This simulation was created using the default orientation and parameter values. 
The time response of the model to a sinusoidal input had a short transient period followed by steady oscillations about the equilibrium point (Figure 3-1). The linear model represented the nonlinear model well over the range of parameters tested and over wrist displacements up to 30 degrees about the equilibrium point (Figure 3-2). The frequency of the sinusoidal input had negligible effect on the error (Figure 3-2).

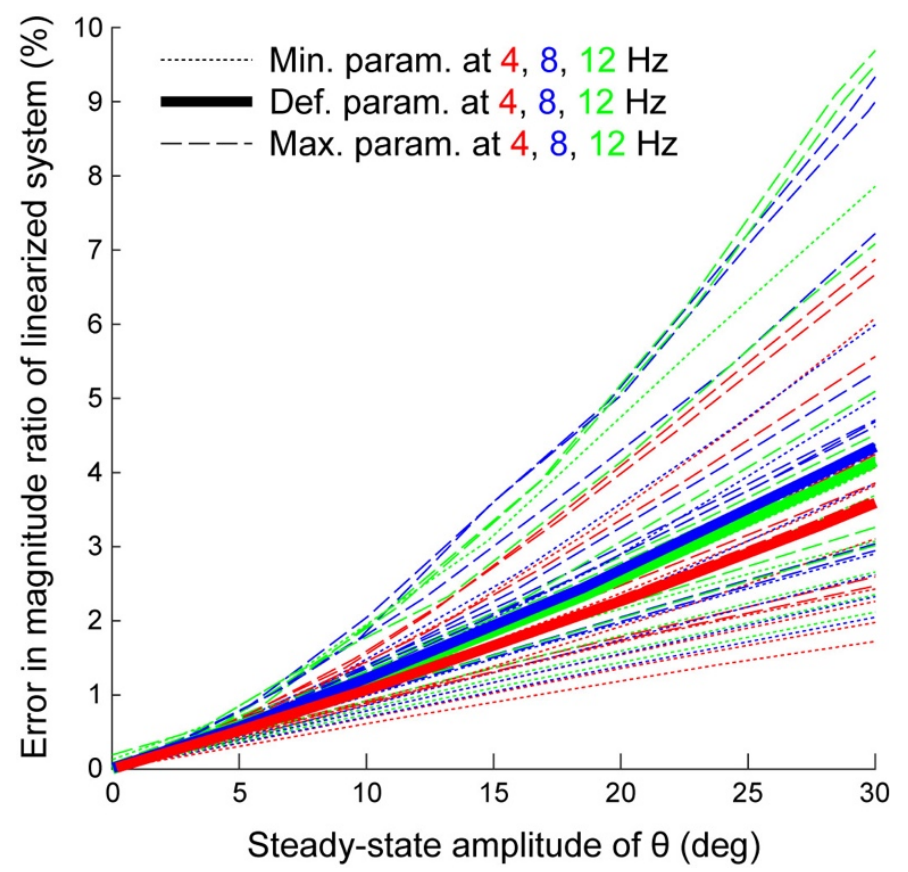

Figure 3-2: Percent error in magnitude ratio between the nonlinear and linearized models as a function of the steady-state amplitude of wrist displacement $(\theta)$, evaluated at input frequencies of 4,8 , and $12 \mathrm{~Hz}$. To validate the linearization over the entire parameter space the magnitude ratio was calculated for different parameter sets. One parameter was varied at a time and set to its default value (thick solid line), its minimum value (dotted line) and then its maximum value (dashed line).

\subsection{Simulations}

\subsubsection{Basic Effects}

As mentioned above, the effect of the gyroscope on tremor can be broken into two parts: the effect caused by the mass of the gyroscope when the gyroscope is turned off and the additional 

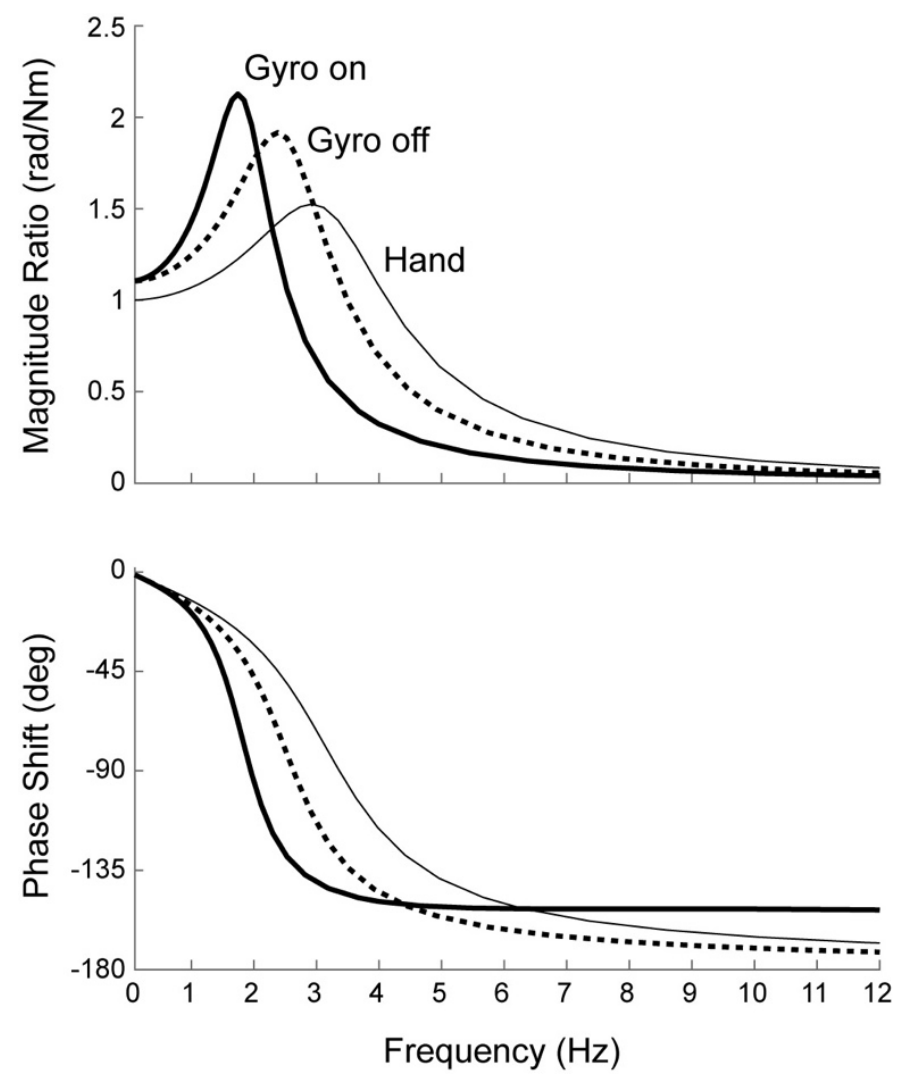

Figure 3-3: Frequency response of the default system (default orientation and parameter values) with the gyroscope in the on-state (thick solid) and off-state (thick dashed), compared to the response of the hand without gyroscope (thin solid). Adding the gyroscope (in the off-state) to the hand produces the usual low-pass filtering effect associated with increased mass, decreasing the peak frequency and bandwidth. Turning the gyroscope on serves to intensify this low-pass filtering effect, further decreasing the peak frequency and bandwidth of the system.

effect caused by the spinning flywheel when the gyroscope is turned on (Figure 3-3). For the default configuration and the default parameters, adding the gyroscope in the off-state and then turning the gyroscope on decreased the peak frequency of the magnitude ratio from $2.8 \mathrm{~Hz}$ (hand only) to $2.3 \mathrm{~Hz}$ (gyro off) to $1.7 \mathrm{~Hz}$ (gyro on) and increased the peak amplitude of the magnitude ratio from 1.4 (hand only) to 1.8 (gyro off) to 2.0 (gyro on). The bandwidth decreased from $4.7 \mathrm{~Hz}$ (hand only) to $3.8 \mathrm{~Hz}$ (gyro off) to $2.8 \mathrm{~Hz}$ (gyro on). Adding the gyroscope in the off-state and then turning it on pushed the phase shift to the left; this caused a significant increase in phase shift (in the negative direction) at low frequencies associated with voluntary movement (e.g. at $2 \mathrm{~Hz}$ ) 
but did not have a significant effect on phase shift in the tremor band $(4-12 \mathrm{~Hz})$. In summary, adding the gyroscope in the off-state results in the typical low-pass filtering effect associated with increased mass: decrease in peak frequency and bandwidth and increase in peak amplitude. Hereafter we will refer to this effect as the "mass effect." Turning the gyroscope on appears to further decrease the peak frequency and bandwidth and usually increase the peak amplitude (hereafter referred to as the "spin effect"). The total gyroscope effect is the combination of the mass and spin effects.

\subsubsection{Effect of Individual Parameters on Basic Effect}

Precession axis parameters: Increasing $x_{p}$ or $y_{p}$ increased the distance from the wrist joint axis to the gyroscope (i.e. to the intersection of the precession and spin axes), which increased the mass effect and decreased the spin effect (Figure 3-4). The spin effect can be seen in Figure 3-4 as the difference between the frequency response of the gyroscope in the off-state and the frequency response of the gyroscope in the on-state. To understand why increasing the distance from the wrist joint axis to the gyroscope decreased the spin effect, note the following. Increasing the distance from the wrist joint axis to the gyroscope does not affect the torque produced by the gyroscope, but it does increase the total inertia about the wrist joint axis. Increased inertia decreases the effect the gyroscopic torque has on the hand/gyroscope system, so the spin effect is decreased. However, the increase in the mass effect dominated over the decrease in the spin effect, causing the total gyroscope effect to increase with distance. Conversely, moving the gyroscope closer to the wrist axis made the response look more and more like that of the hand alone. Increasing $z_{p}$ did not affect the distance from the precession axis to the gyroscope and therefore did not affect the mass or spin effects. Increasing $K_{p}$ and $D_{p}$ obviously did not affect the mass 
effect but decreased the spin effect (Figure 3-4). Although this trend held for the entire range of $K_{p}$, it did not for $D_{p}$; whereas large values of $D_{p}$ caused the frequency response to approach that of the gyroscope in the off-state, decreasing $D_{p}$ beyond $0.5 \mathrm{Nms} / \mathrm{rad}$ had little effect on the peak frequency or bandwidth.

Motor Parameters: Varying the four gyroscope motor parameters within the predefined range (Table 2-1) only negligibly affected the mass effect, spin effect, or total gyroscope effect (Figure 3-5).

A
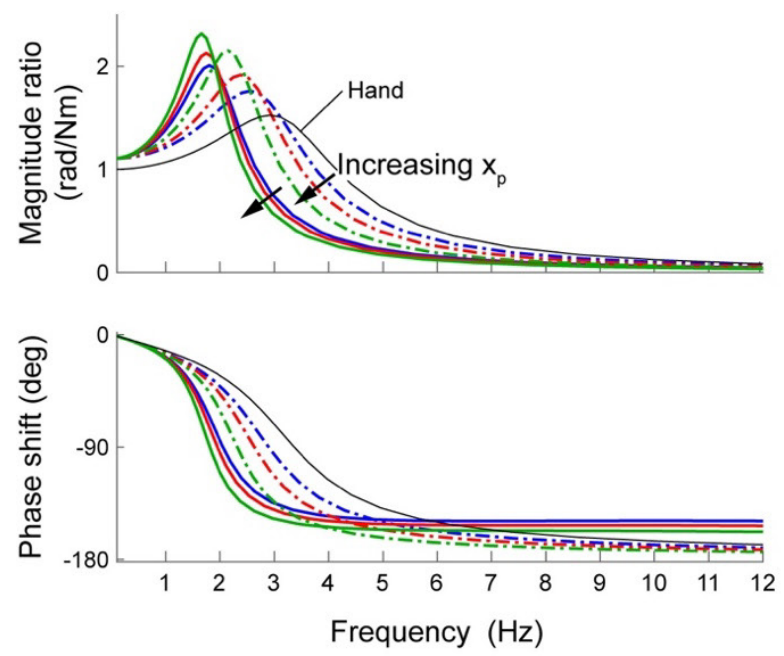

C
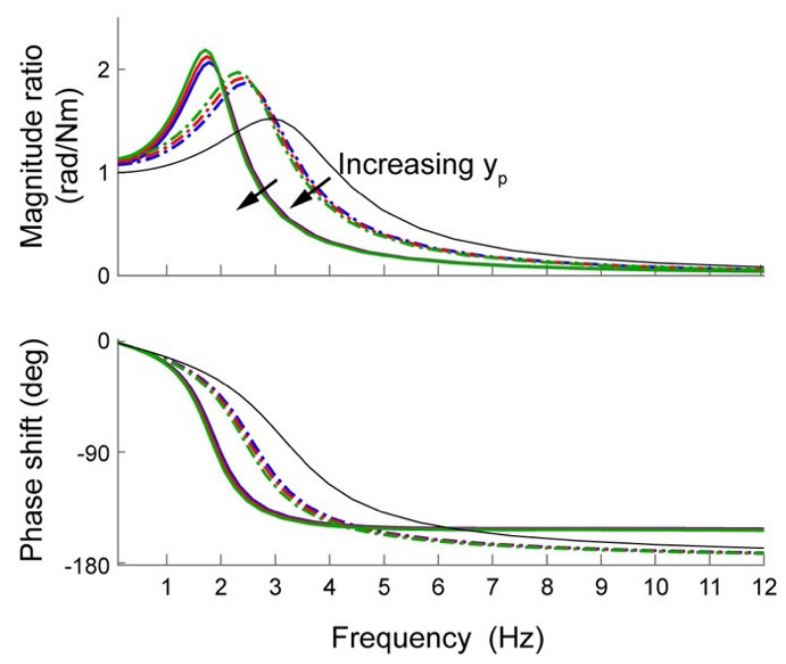

Figure 3-4 continues on next page
B
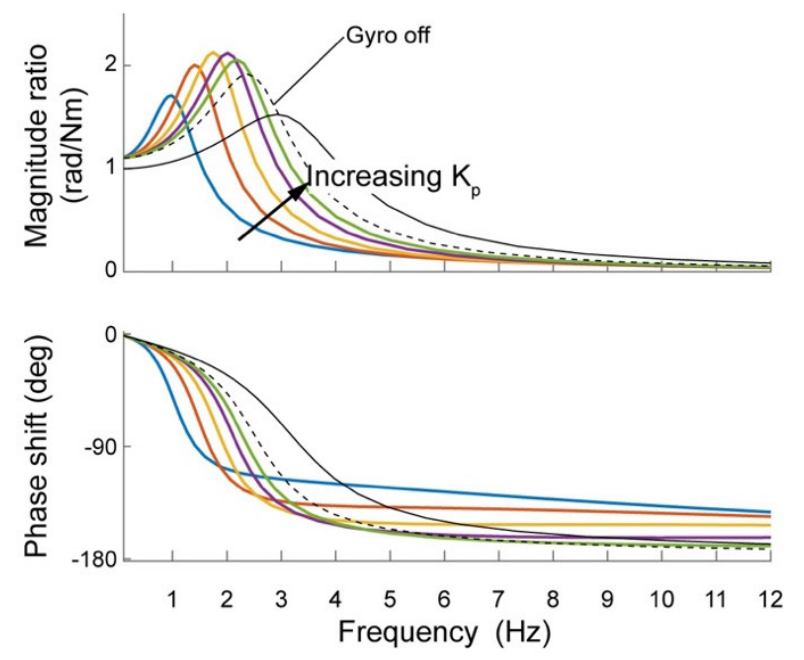

D
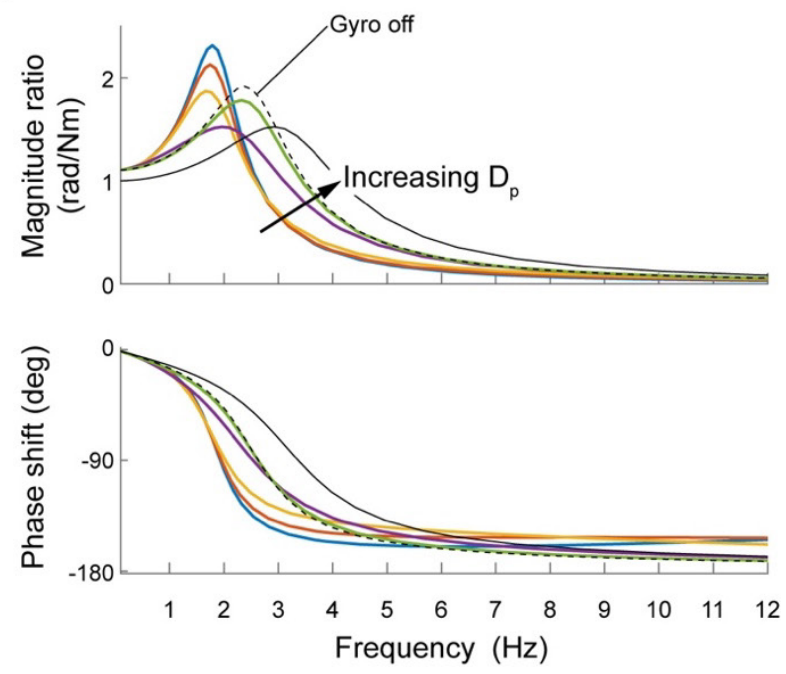

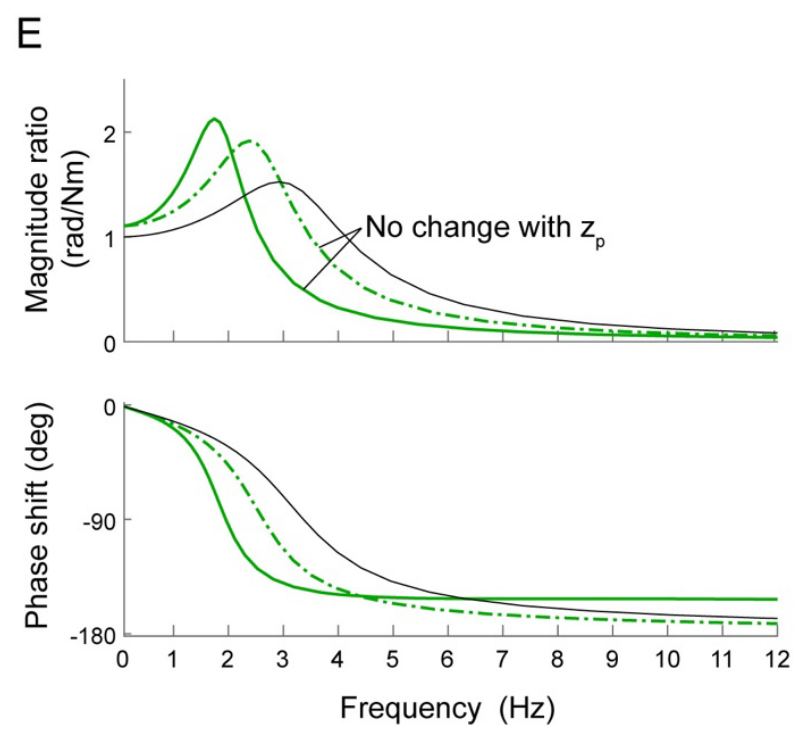

Figure 3-4: Effect of varying precession axis parameters (Table 2-1) on the frequency response of the default system in the on-state (thick solid) and off-state (thick dashed). Also included for reference is the response of the hand without the gyroscope (thin solid). Subplots depict the influence of the distance from the wrist joint axis to the gyroscope $\left(x_{p}, y_{p}\right.$, and $z_{p}$ depicted in subplots $\mathrm{A}, \mathrm{C}$, and $\mathrm{E}$, respectively) as well as the precession axis stiffness $K_{p}$ and damping $D_{p}$ (subplots $\mathrm{B}$ and D). In the default configuration (Figure 2-1), changing $z_{p}$ merely moves the gyroscope parallel to the wrist joint axis and therefore has no effect, as seen in subplot E. The frequency response of the hand by itself (without gyroscope) is the same in each plot because the precession axis parameters have no effect on the hand. Similarly, the frequency response of the gyroscope in the off-state is the same in B and D because the precession stiffness and damping has no effect on the gyroscope in the off-state.

Flywheel Parameters: Increasing $R_{f}, L_{f}$, or $\rho_{f}$ increased the inertia of the flywheel about the spin axis, which increased both the mass and spin effects (Figure 3-6). Increasing $r_{f}$ from negative to positive increased the mass effect and decreased the spin effect slightly (note that negative values of $r_{f}$ indicate that the center of mass of the gyroscope lies on the negative $\mathbf{y}$-axis, and positive values of $r_{f}$ indicate that the center of mass of the gyroscope lies on the positive $\mathbf{y}$ axis). Increasing $r_{f}$ had a similar effect as increasing $y_{p}$; increasing $r_{f}$ moved the flywheel farther from the hand (Figure 2-1), which increased the inertia about the wrist axis, increasing the mass effect. This increased inertia caused the gyroscope to have a smaller effect, decreasing the spin effect. By keeping the gyroscope close to its equilibrium position, the stiffness about the precession 
axis reduced the effect of gravity and removed the instability that a positive offset would have otherwise caused. Increasing $\Omega$ obviously did not affect the mass effect but greatly increased the spin effect. The trends from varying the gyroscope parameters are concisely summarized in Table $3-1$.

A
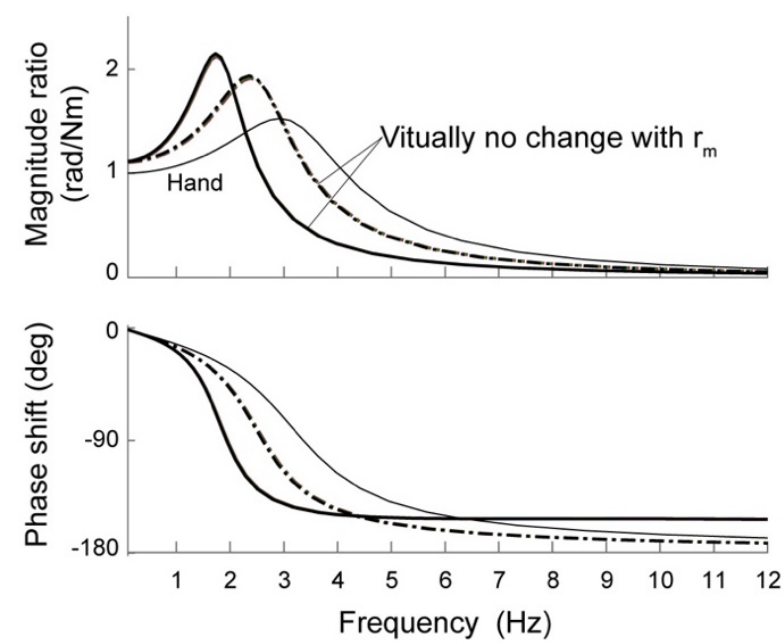

C
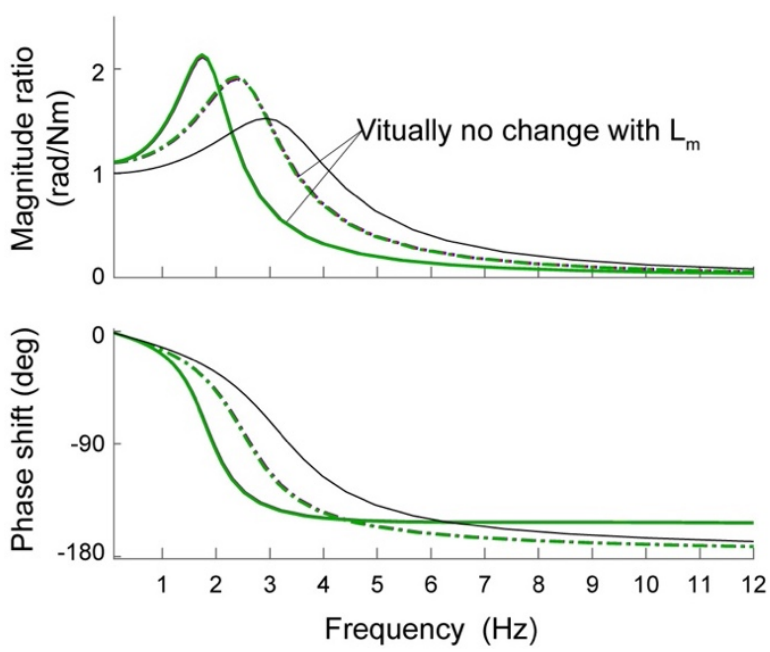

B
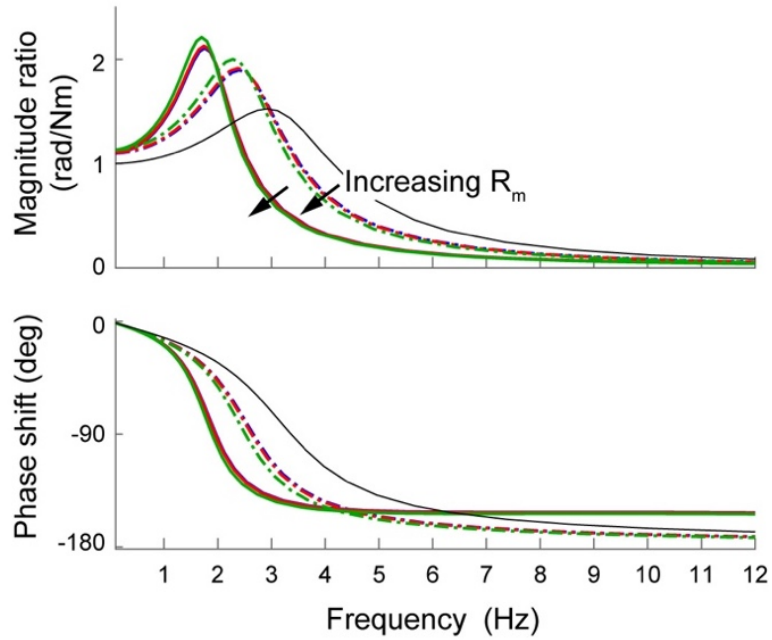

D
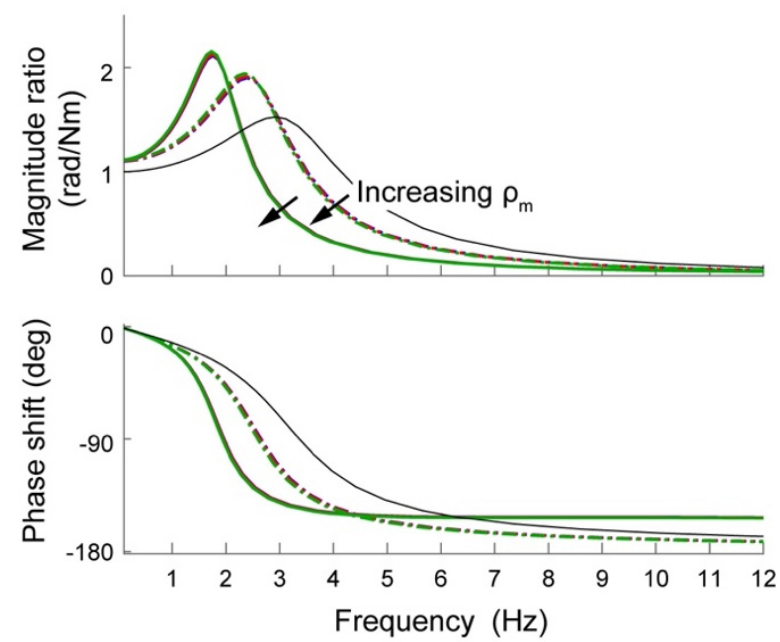

Figure 3-5: Varying the motor parameters (Table 2-1) has a negligible effect on the frequency response of the default system in the on-state (thick solid) and off-state (thick dashed). For reference, the response of the hand without the gyroscope is also included (thin solid). Subplots depict the influence of the offset of the motor $r_{m}$ from the precession axis to the center of mass of the motor (A), the radius $R_{m}$ and length $L_{m}$ of the cylindrically shaped motor (B and C, respectively), and the density $\rho_{m}$ of the motor (D). The frequency response of the hand is the same in each subplot because varying the motor parameters has no effect on the hand. 


\subsubsection{Robustness to variations in wrist-hand parameters and gravity}

These results were obtained from simulations using average hand and wrist parameters. The center of mass, mass, and inertia of the hand represented an average of male and female subjects, and the stiffness and damping of the wrist joint represented passive muscle (i.e. in the absence of muscle contraction). Changing the mass/inertia parameters to represent either male or female subjects, and changing the joint stiffness and damping to represent various states of muscle activation did not change any of the trends described above (Table 3-1). In other words, these

A
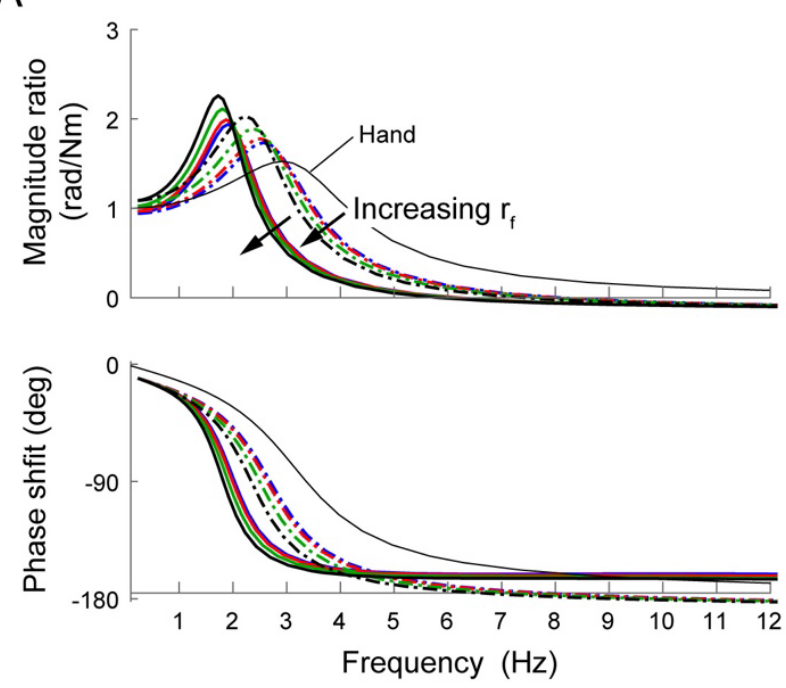

C
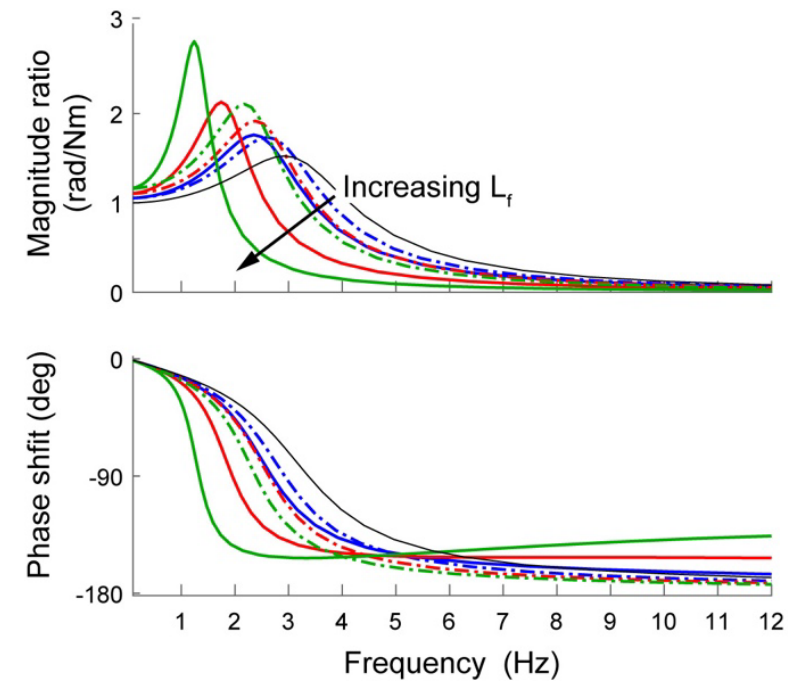

Figure 3-6 continues on next page
B
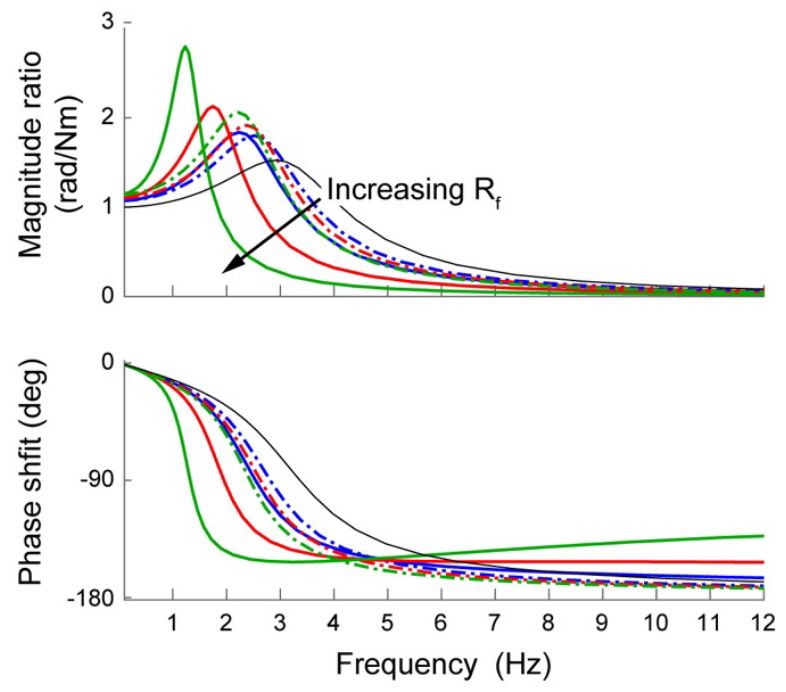

D
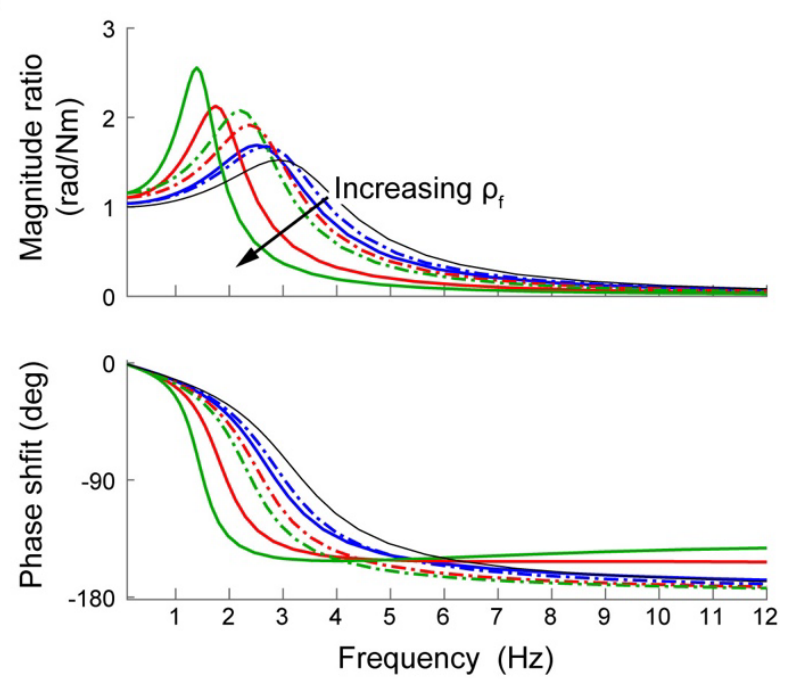


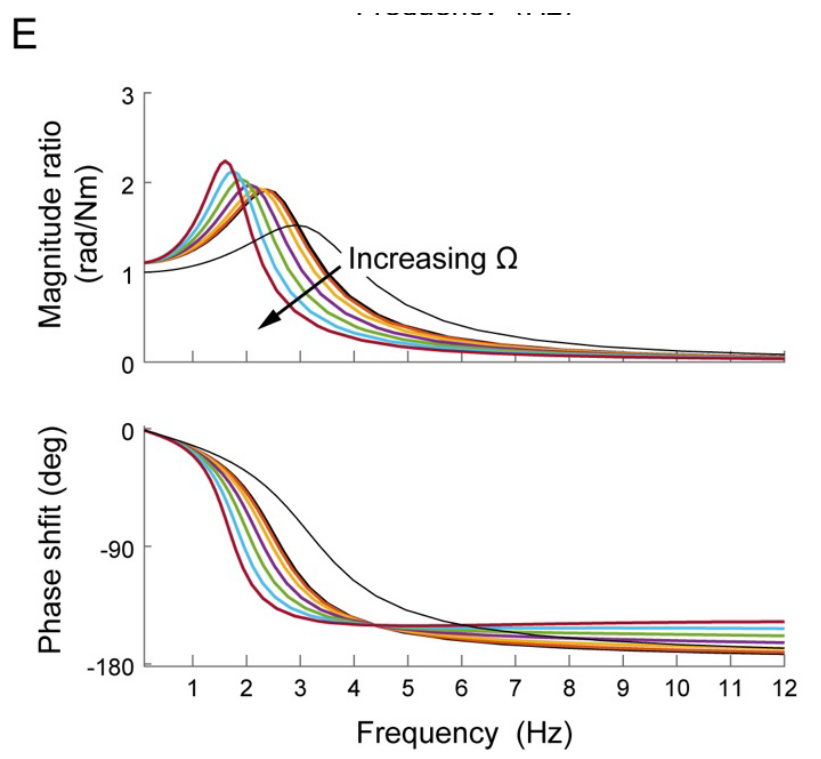

Figure 3-6: Effect of varying flywheel parameters (Table 2-1) on the frequency response of the default system in the on-state (thick solid) and off-state (thick dashed), compared to the response of the hand without the gyroscope (thin solid). Subplots depict the influence of the offset of the flywheel $r_{f}$ from the precession axis to the center of mass of the flywheel (A), the radius $R_{f}$ and length $L_{f}$ of the cylindrically shaped flywheel (B and C), and the density $\rho_{f}$ and spin speed $\Omega$ of the flywheel ( $\mathrm{D}$ and $\mathrm{E}$ ). The frequency response of the hand is the same in each subplot because varying flywheel parameters has zero effect on the hand.

trends are robust to changes in hand and wrist parameters (within the ranges listed in Table 2-1). Similarly, removing gravity from the simulation did not change any of the results described above either, indicating that the trends are also robust to changes in gravity.

\subsubsection{Robustness to different configurations}

The LTI state-space models of the other 11 cardinal configurations (Figure 2-3) were found to approximate the nonlinear EOM well, similar to the default system (Figure 3-1 and Figure 3-2). Five of these 11 cardinal configurations behaved similarly to the default configuration (FE O2): FE O4, RUD O3, RUD O6, PS O1, and PS O5 (Figure 2-3). Together with the default configuration, these six configurations are the cardinal configurations in which the wrist joint axis, precession axis, and spin axis are mutually perpendicular. There were slight differences in the dynamics of 
Table 3-1: This table summarizes the difference in frequency response (input $=$ wrist torque, output=wrist angle) between "hand without gyroscope" and "hand with gyroscope-in-offstate" (mass effect), "hand with gyroscope-in-off-state" and "hand with gyroscope-in-onstate" (spin effect), and "hand without gyroscope" and "hand with gyroscope-in-on-state"

(Total effect). The symbols --, $\sim, \uparrow, \downarrow, \sim \uparrow$, and $\sim \downarrow$ mean there was no effect, negligible effect, increased effect, decreased effect, slight increase, and slight decrease, respectively. A comma means that the results change, for example, $\sim \uparrow$ means the response is negligible and then it increases.

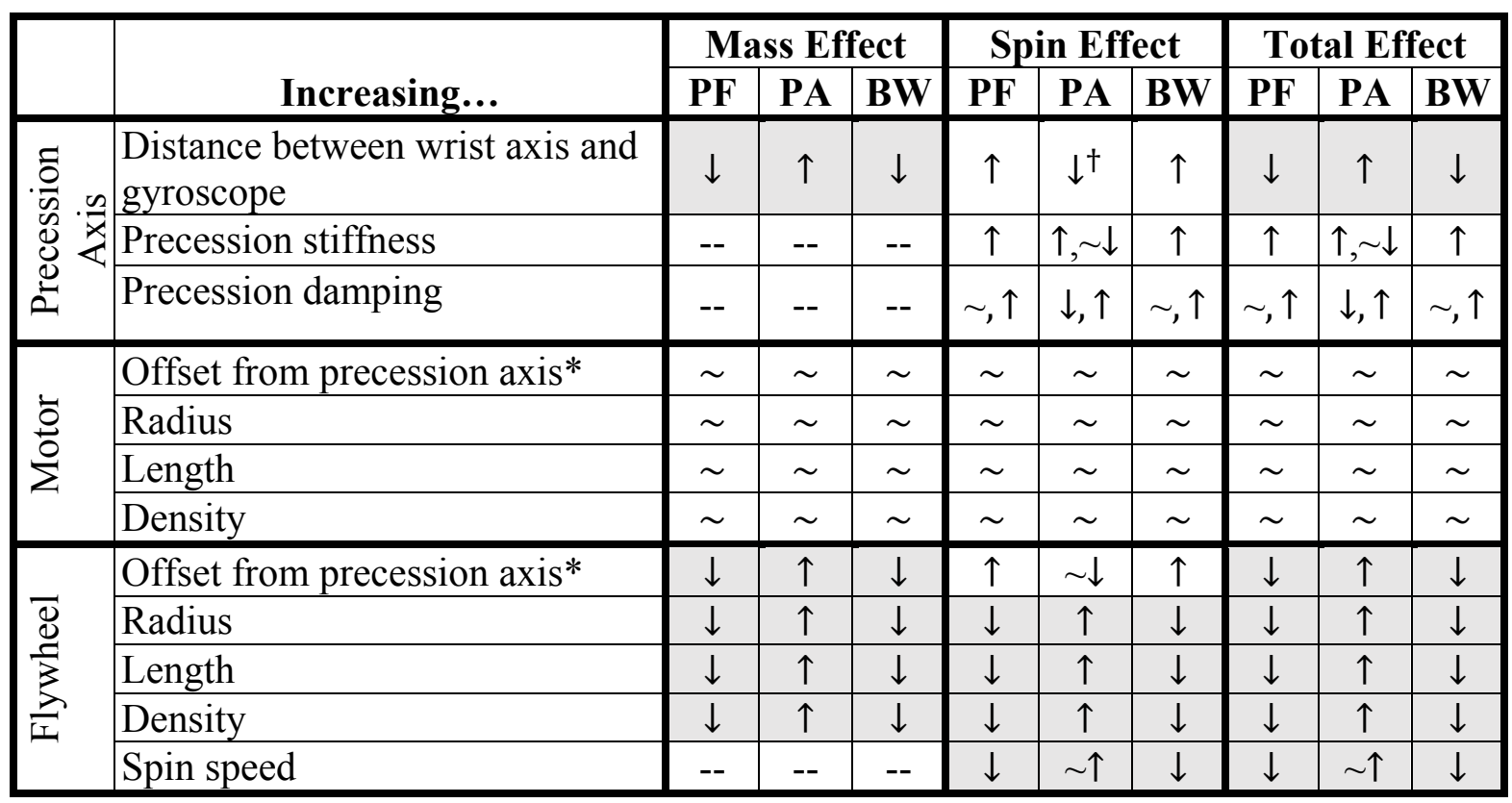

* This offset is the signed offset for O1-O4 and the unsigned offset for O5-O6. Increasing the offset for O1-O4 always increased the mass effect. For O5-O6, zero offset was the minimum, i.e. any offset increased the mass effect.

$\dagger$ Increasing $y_{p}$ caused the peak amplitude to slightly increase.

these six configurations; the three wrist joint axes (FE, RUD, and PS) experienced slightly different inertias, and the associated gyroscopes had slightly different dynamics (because the direction in which the gyroscope was offset from the precession axis depended on the wrist joint $\left.\operatorname{axis}^{3}\right)$. Nevertheless, despite these minor differences, these six configurations exhibited the same trends when the gyroscope parameters or hand-wrist parameters were varied, or when gravity was

${ }^{3}$ The direction in which the gyroscope was offset from the precession axis was: $\mathbf{y}_{1}$-axis for O1 and $\mathrm{O} 2, \mathbf{x}_{1}$-axis for $\mathrm{O} 3$ and $\mathrm{O} 4$, and $\mathbf{z}_{1}$-axis for $\mathrm{O} 5$ and O6. Therefore, varying $r_{f}$ had a similar effect as varying the distance between the wrist joint axis and gyroscope in the direction parallel to $r_{f}$ $\left(y_{p}\right.$ for $\mathrm{O} 1$ and $\mathrm{O} 2, x_{p}$ for $\mathrm{O} 3$ and $\mathrm{O} 4$, and $z_{p}$ for $\mathrm{O} 5$ and $\mathrm{O} 6$ ). 
altered. Therefore, within these six cardinal configurations, the trends listed above (Table 3-1) were robust to changes in configuration.

In contrast, the other 6 cardinal configurations had negligible spin effect as expected. When the wrist joint axis was parallel to the spin axis but not parallel to the precession axis (FE O5, FE O6, RUD O1, RUD O2, PS O3, and PS O4), wrist joint movement did induce a torque on the gyroscope, but the induced torque acted about the axis orthogonal to the spin and the precession axes (and therefore primarily orthogonal to the wrist joint axis), with only a small spin effect about the wrist joint axis. 


\section{DISCUSSION}

Here we present a basic analysis of gyroscopic tremor suppression in the wrist joint to inform the future development of an optimal gyroscopic tremor suppression device. Reaching optimal gyroscopic tremor suppression requires knowledge of how varying each gyroscopic parameter will influence tremor suppression. This is the first systematic investigation of gyroscopic tremor suppression of which we are aware. Consequently, we deliberately implemented a simple model to focus first on the most basic effects. From these effects, we established the following fundamental principles that govern how the parameters of a gyroscope affect tremor suppression. These principles were observed under specific simulation conditions (See "Limitations" section below), and more research must be performed to generalize outside of these conditions.

\subsection{Linearization}

The range of the wrist and forearm is approximately $\pm 85^{\circ}$ in $\mathrm{PS}, \pm 70^{\circ}$ in $\mathrm{FE}$, and from $25^{\circ}$ in ulnar deviation to $15^{\circ}$ in radial deviation. However, most tremors have relatively small joint displacements. Assuming tremor was focused at the wrist or forearm joint, severe, moderate, and mild tremor is below 28, 15, and 9 deg, respectively [43]. Therefore, the average linearization error of most tremors is less than $9 \%, 4 \%$, and $2 \%$ for severe, moderate, and mild tremor, respectively over the range of parameters tested. This linearization is almost entirely independent of frequency 
and is therefore valid for the entire tremor band. Linearizing allowed us to use tools from linear system theory to analyze the effects of the gyroscope on tremor suppression.

\subsection{Simulation}

\subsubsection{Total Gyroscope Effect}

As mentioned above, the total effect of the gyroscope can be divided into the effect caused by adding the mass of the gyroscope when it is turned off ("mass effect") and the effect of the spinning flywheel caused by turning the gyroscope on ("spin effect"). The mass decreased the frequency and increased the amplitude of the resonance peak, resulting in an overall decrease in bandwidth and the low-pass filtering effect commonly associated with mass. By resisting changes in angular momentum, the spin of the flywheel further decreased the resonance frequency and (usually) increased the amplitude of the resonance peak, resulting in a further decrease in bandwidth. Whereas the mass effect acted no matter the direction of the wrist joint axis, precession axis, or spin axis, the spin effect became negligible if two or more of these three axes were parallel in their equilibrium position. Consequently, only six of the 12 cardinal configurations (Figure 2-3) had a non-negligible spin effect.

The overall decrease in the frequency of the resonance peak was accompanied by an increase in phase shift (more negative) at lower frequencies. For our default parameters, the gyroscope caused the phase shift to practically reach its asymptotic value within the frequency band associated with voluntary movement $(0-3 \mathrm{~Hz})$. This may have an effect on voluntary movement; however, since this effect is similar to the low-pass filtering effect of holding a mass, we expect subjects would adapt quickly to the dynamics of the gyroscope. 
In summary, the total gyroscope effect, which is a combination of the mass and spin effects, is simply a low-pass filter. If the gyroscope parameters are chosen such that its overall bandwidth includes the frequencies of most voluntary movement $(0-3 \mathrm{~Hz})$ but excludes the frequencies of most tremors (4-12 Hz), the gyroscope may be appropriate for tremor suppression. Our simulations revealed how the various gyroscope parameters affected the low-pass filtering properties of the gyroscope, including the bandwidth.

\subsubsection{Precession Axis Parameters}

Increasing the distance of the gyroscope from the wrist joint axis increased the inertia and therefore the mass effect of the gyroscope. In contrast, moving the gyroscope parallel to the wrist joint axis had no effect on the inertia or mass effect. Although the mass effect by itself low-pass filters, it obviously comes at the cost of increased mass and inertia about the wrist. Interestingly, changing the location of the gyroscope has no effect on the angular momentum of the gyroscope; consequently, the restoring torque produced by the gyroscope is independent of its location on the hand. That said, increasing the distance of the gyroscope from the wrist joint axis increases its inertia, which decreases the ability of the restoring torques to affect the hand. Therefore, by placing the gyroscope closer to the wrist joint axis, it may be possible to achieve the same overall lowpass filtering effect as placing the gyroscope farther away, but with reduced mass and inertia.

Precession stiffness produces the equilibrium torque needed to keep the gyroscope close to its equilibrium position despite changes in orientation and gravitational torque, as well as to simulate the limited range of motion (of the gyroscope about the precession axis) inherent in most gyrostabilizer designs. Precession damping represents the natural friction present in realizable systems. Together, precession stiffness and damping resist precession of the gyroscope, which 
decreases the gyroscope's torque and hence the spin effect. In the limit, increasing the precession stiffness and damping causes the frequency response to approach that of the gyroscope when it is turned off. Note that increasing the precession damping from intermediate to high values first reduced the amplitude of the resonance peak (away from the gyroscope in the off-state) before increasing the amplitude of the resonance peak (toward the gyroscope in the off-state), as shown in Figure 3-4. Overall, to maximize tremor suppression, precession stiffness and damping should be kept low to keep the bandwidth low.

\subsubsection{Flywheel Parameters}

Increasing the radius, length, or density of the flywheel increased the inertia of the flywheel about the wrist joint axis, which increased the mass effect. Increasing the radius, length, or density of the flywheel also increased the inertia and therefore the momentum of the flywheel about the spin axis, which increased the spin effect. As a result, the total gyroscope effect increased greatly. Note that this effect is different from that of increasing the distance of the gyroscope from the wrist joint axis; although increasing the distance also increased the total inertia of the gyroscope about the wrist joint axis, resulting in an increase in the mass effect, it decreased the spin effect. Consequently, the total gyroscope effect increased only moderately. Therefore, increasing the mass of the flywheel is more efficient than placing the gyroscope farther from the wrist joint axis.

Increasing the signed offset of the center of mass of the flywheel from the precession axis, from closer to the hand to farther, slightly increased the mass effect, which caused the spin effect

to slightly decrease. Overall, the total gyroscopic effect increased slightly. Since the precession stiffness has a larger effect than the flywheel offset and it is preferential to keep the stiffness low, 
the flywheel offset should be as close to 0 as possible since this will allow the precession stiffness to be decreased (the stiffness provides an equilibrium torque).

Increasing the spin speed obviously did not affect the mass effect but increased the angular momentum of the flywheel and therefore also the restoring torque and the spin effect, resulting in an increase in the total gyroscopic effect. As increasing the spin speed does not increase mass or inertia about the wrist joint axis, it is an efficient way to increase the total gyroscopic effect.

\subsubsection{Motor Parameters}

Varying the motor parameters had only a negligible effect because the mass of the motor was negligible compared to the mass of the flywheel. For the default parameters, the mass of the motor $(0.035 \mathrm{~kg})$ was only $8 \%$ of the mass of the flywheel $(0.44 \mathrm{~kg})$. If the mass were larger, the effect would be the same as the flywheel mass effect.

\subsubsection{Robustness}

These trends were unaffected by changes in hand and wrist parameters, the orientation of the gyroscope (as long as the wrist joint axis, precession axis, and spin axis were mutually perpendicular), and the presence or absence of gravity. Gravity only had a negligible effect because the precession stiffness limited the amount of precession and provided an equilibrium torque to counteract the gravitational torque about the precession axis. We focused on tremor between 4 and $12 \mathrm{~Hz}$, which covers most tremor types [42]. Therefore, the trends listed above apply to suppressing most types of tremor in a single DOF at the wrist (FE or RUD) or forearm (PS), no matter what the orientation of the arm is with respect to gravity. 


\subsection{Comparisons to Prior Studies}

This is the first systematic investigation of gyroscopic tremor suppression of which we are aware. However, a prior review of gyrostabilizer vehicular technology characterized a wide range of gyrostabilizer systems, reviewed the history of gyrostabilizers, and presented a derivation of the governing equations of motion for some example applications [30]. This review confirmed that increasing the speed and inertia of the gyroscope flywheel increased the spin effect and, consistent with our findings, found that linear equations of motion provided an adequate approximation to the non-linear equations of motion when the precession of the gyroscope was limited to rotating about an equilibrium point. In a study on control strategies for marine gyrostabilizers, Townsend found that passive stabilization (i.e. without any active control torque on the precession axis) led to motion attenuation at higher frequencies and motion amplification at lower frequencies [31]. Likewise, we focused here on passive stabilization and found consistent effects (Figure 3-3). Unfortunately, other studies of gyrostabilization generally focused on active stabilization (e.g. of vibrations in structures [44] or gondolas [45]) and did not methodically explore the effect of parameters on passive stabilization, let alone in the context of tremor suppression.

\subsection{Limitations}

As mentioned above, we deliberately chose a simple model to establish the most basic principles. Our model is linear time-invariant and only includes a single skeletal DOF (either FE, RUD, or PS) and a single gyroscope DOF (precession). To analyze the effect of the gyroscope on tremor, we used standard frequency response techniques, which focus on the steady-state response to sinusoidal inputs. Therefore, our simulations ignored the following effects: time-varying wrist impedance (stiffness and damping) and spin speed, transient responses, and reflexes. More work is needed to characterize how these factors affect gyroscopic tremor suppression. Likewise, the 
model should be expanded to include more skeletal DOF (e.g. FE, RUD, and PS, and eventually all seven DOF from the shoulder to the wrist) to investigate the side effects of gyrostabilization on volitional movement (this cannot be studied using a 1-DOF model) and determine optimal gyroscope parameters (including the number and location of gyroscopes) for the entire upper limb. Additionally, we did not look at the interaction effects of the parameters by varying multiple parameters at the same time instead of just varying one parameter at a time. Our simulations also assumed the forearm was grounded and therefore ignored the effect of upper-limb movement on the gyroscope and (secondarily) on tremor suppression. Lastly, the principles presented here were based on simulations and were not validated with experimental data. Future studies should include experimental validation.

\subsection{Conclusion}

Using a simple model of a gyroscope mounted on the back of a hand tremoring about a single wrist joint axis, we have established the fundamental effects of gyroscope parameters on tremor suppression. To the best of our knowledge, this is the first systematic investigation of gyroscopic tremor suppression. We found that gyroscopes decrease the bandwidth of the frequency response and can therefore be described as low-pass filters. To minimize the bandwidth without adding too much inertia about the wrist joint, the inertia and spin speed of the flywheel should be as high as design constraints allow, whereas the distance from the wrist joint axis to the gyroscope and precession stiffness should be kept as low as design constraints allow. Decreasing the precession damping beyond its minimum point will increase the order of the low-pass filtering effect, leading to increased tremor suppression at the cost of a higher peak resonance amplitude. 
Placing the center of mass of the flywheel and motor on the precession axis $\left(r_{f}=r_{m}=0\right)$ would reduce the effect of gravity and allow the precession stiffness to be low.
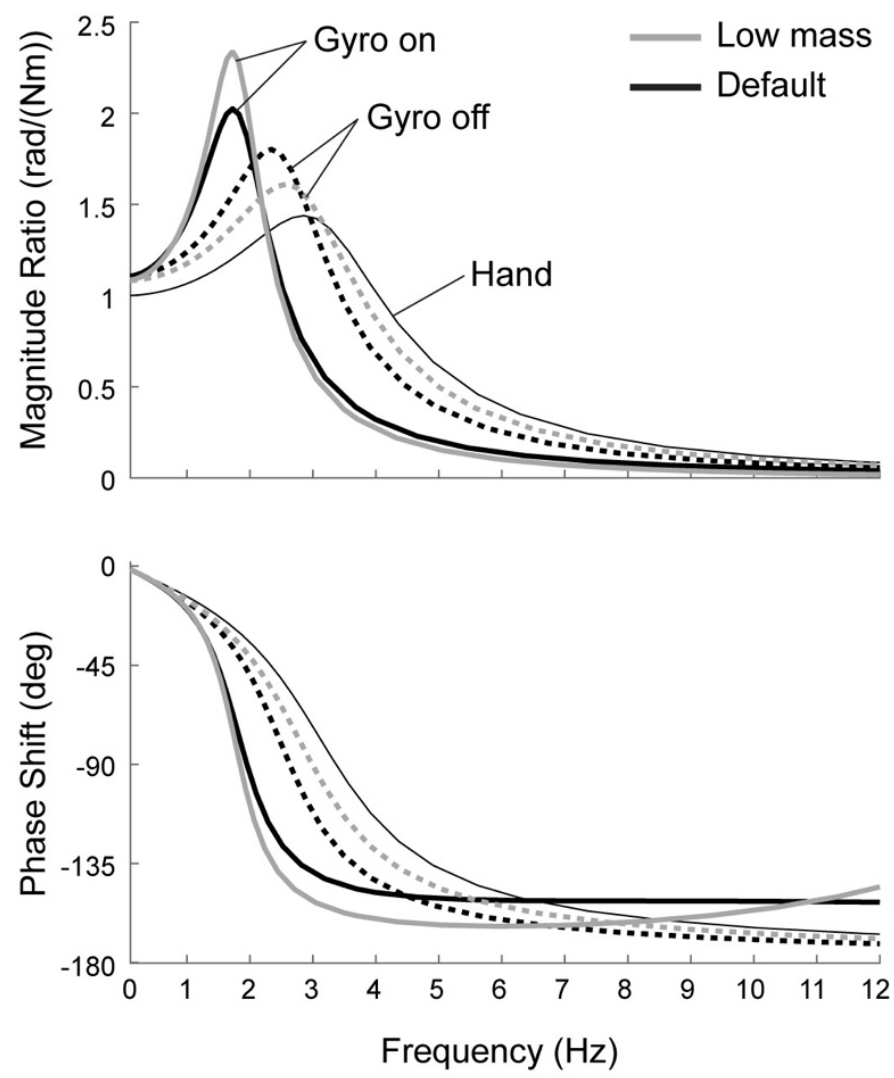

Figure 4-1: Frequency response of the default configurations using the default parameters with the gyroscope in the on-state (solid blue) and off-state (solid red), versus the frequency response using more optimal parameters with the gyroscope in the on-state (dashed blue) and off-state (dashed red), compared to the response of the hand without the gyroscope (black). Adding the gyroscope in the off-state had a much smaller effect when the optimal parameters were used, however the optimal parameters led to a slightly increased effect when the gyroscope is in the on-state.

A more optimal set of parameters were created by setting $r_{f}=r_{m}=0, x_{p}$ and $K_{p}$ to their smallest values in Table 2-1, and $\Omega$ to its largest value in Table 2-1. To decrease the mass effect the length of the flywheel was set at $1 \mathrm{~cm}$. Additionally, the flywheel was changed from a cylinder into an annulus with an inner radius of $1.5 \mathrm{~cm}$, to further decrease the mass of the flywheel while having a smaller change on the flywheel inertia about the spin axis. After these changes to the flywheel the mass decreased from $0.44 \mathrm{~kg}$ with the default parameters to $0.17 \mathrm{~kg}$ with the optimal 
parameters. Lastly, $D_{p}$ was decreased until the magnitude ratio was sufficiently low in the tremor band, which occurred with a precession damping of $0.005 \mathrm{Nms} / \mathrm{rad}$. The resulting frequency response to this more optimal parameter set strongly show the potential of a gyroscopic tremor suppression device (Figure 4-1).

For both the default and optimal parameter sets the peak frequency of the magnitude ratio, the peak amplitude of the magnitude ratio, and the bandwidth of the hand alone were $2.8 \mathrm{~Hz}, 1.4$, and $4.7 \mathrm{~Hz}$, respectively. Adding the gyroscope with no spin speed caused the peak frequency to decrease to $2.3 \mathrm{~Hz}$ (default) and $2.5 \mathrm{~Hz}$ (optimal), the peak amplitude to increase to 1.8 (default) and 1.6 (optimal), and the bandwidth to decrease to $3.8 \mathrm{~Hz}$ (default) and $4.2 \mathrm{~Hz}$ (optimal). Turning on the gyroscope cause the peak frequency to further decrease to $1.7 \mathrm{~Hz}$ (default and optimal), the peak amplitude to further increase to 2.0 (default) and 2.3 (optimal), and the bandwidth to further decrease to 2.8 (default) and $2.7 \mathrm{~Hz}$ (optimal). This one example shows how varying the gyroscopic parameters can have significant effects on the resulting response of the hand. The numbers show that the optimal parameters led to a relatively large decrease in the mass effect, while improving the total gyroscope effect slightly, indicating a much larger increase in the spin effect. Future studies should focus on performing a more rigorous optimization of parameters with the goal of obtaining a flatter pass band, a cutoff frequency around $3 \mathrm{~Hz}$ (the end of volitional movement), and a minimal addition of mass. We expect the principles in this thesis to serve as a foundation for more sophisticated models of gyroscopic tremor suppression in the upper limb and as a basis for developing effective gyroscopic tremor-suppressing devices. 


\section{REFERENCES}

[1] A. Anouti and W. C. Koller, "TREMOR DISORDERS - DIAGNOSIS AND MANAGEMENT," (in English), Western Journal of Medicine, Article vol. 162, no. 6, pp. 510-513, Jun 1995.

[2] E. Rocon, J. M. Belda-Lois, J. J. Sanchez-Lacuesta, and J. L. Pons, "Pathological tremor management: Modelling, compensatory technology and evaluation," Technology \& Disability, Article vol. 16, no. 1, pp. 3-18, 2004.

[3] A. D. Davidson and S. K. Charles, "Fundamental Principles of Tremor Propagation in the Upper Limb," (in English), Annals of Biomedical Engineering, Article vol. 45, no. 4, pp. 1133-1147, Apr 2017.

[4] G. K. Wenning et al., "Prevalence of movement disorders in men and women aged 50-89 years (Bruneck Study cohort): a population-based study," (in English), Lancet Neurology, Article vol. 4, no. 12, pp. 815-820, Dec 2005.

[5] K. E. Lyons and R. Pahwa, "Pharmacotherapy of Essential Tremor An Overview of Existing and Upcoming Agents," (in English), Cns Drugs, Review vol. 22, no. 12, pp. 1037-1045, 2008.

[6] D. Kondziolka, J. G. Ong, J. Y. K. Lee, R. Y. Moore, J. C. Flickinger, and L. D. Lunsford, "Gamma Knife thalamotomy for essential tremor," (in English), Journal of Neurosurgery, Article vol. 108, no. 1, pp. 111-117, Jan 2008.

[7] S. K. Kalia, T. Sankar, and A. M. Lozano, "Deep brain stimulation for Parkinson's disease and other movement disorders," (in English), Current Opinion in Neurology, Review vol. 26, no. 4, pp. 374-380, Aug 2013.

[8] E. D. Louis, "Essential tremor," (in English), Lancet Neurology, Review vol. 4, no. 2, pp. 100-110, Feb 2005.

[9] T. A. Zesiewicz et al., "Practice parameter: Therapies for essential tremor - Report of the quality standards subcommittee of the American Academy of Neurology," (in English), Neurology, Article vol. 64, no. 12, pp. 2008-2020, Jun 2005.

[10] J. Jankovic, "Complications and limitations of drug therapy for Parkinson's disease," Neurology, vol. 55, no. 12, p. S2-S6, 2000.

[11] J. Jankovic and A. L. G., "Current approaches to the treatment of Parkinson's disease," Neuropsychiatr Dis Treat, vol. 4, no. 4, pp. 743-757, 2008. 
[12] S. D. Piasecki and J. W. Jefferson, "Psychiatric complications of deep brain stimulation for Parkinson's disease," (in English), Journal of Clinical Psychiatry, Article vol. 65, no. 6, pp. 845-849, Jun 2004.

[13] F. Vergani, A. Landi, D. Pirillo, R. Cilia, A. Antonini, and E. P. Sganzerla, "Surgical, Medical, and Hardware Adverse Events in a Series of 141 Patients Undergoing Subthalamic Deep Brain Stimulation for Parkinson Disease," (in English), World Neurosurgery, Article vol. 73, no. 4, pp. 338-344, Apr 2010.

[14] J. Kotovsky and M. J. Rosen, "A wearable tremor-suppression orthosis," (in English), Journal of Rehabilitation Research and Development, Article vol. 35, no. 4, pp. 373-387, Oct 1998.

[15] S. M. Hashemi, M. F. Golnaraghi, and A. E. Patla, "Tuned vibration absorber for suppression of rest tremor in Parkinson's disease," (in English), Medical \& Biological Engineering \& Computing, Article vol. 42, no. 1, pp. 61-70, Jan 2004.

[16] J. M. Belda-Lois et al., "Controllable mechanical tremor reduction. Assessment of two orthoses," Technology \& Disability, Article vol. 19, no. 4, pp. 169-178, 2007.

[17] R. C. V. Loureiro, J. M. Belda-Lois, E. R. Lima, J. L. Pons, J. J. Sanchez-Lacuesta, and W. S. Harwin, "Upper limb tremor suppression in ADL via an orthosis incorporating a controllable double viscous beam actuator," in Rehabilitation Robotics, 2005. ICORR 2005. 9th International Conference on, 2005, pp. 119-122.

[18] D. Case, B. Taheri, and E. Richer, "Design and Characterization of a Small-Scale Magnetorheological Damper for Tremor Suppression," (in English), Ieee-Asme Transactions on Mechatronics, Article vol. 18, no. 1, pp. 96-103, Feb 2013.

[19] D. Case, B. Taheri, and E. Richer, "A Lumped-Parameter Model for Adaptive Dynamic MR Damper Control," (in English), Ieee-Asme Transactions on Mechatronics, Article vol. 20, no. 4, pp. 1689-1696, Aug 2015.

[20] D. Case, B. Taheri, and E. Richer, "Dynamical Modeling and Experimental Study of a Small-Scale Magnetorheological Damper," (in English), Ieee-Asme Transactions on Mechatronics, Article vol. 19, no. 3, pp. 1015-1024, Jun 2014.

[21] M. J. Rosen, A. S. Arnold, I. J. Baiges, M. L. Aisen, and S. R. Eglowstein, "Design of a Controlled-Energy-Dissipation Orthosis (CEDO) for Functional Suppression of Intention Tremors," (in English), Journal of Rehabilitation Research and Development, Article vol. 32, no. 1, pp. 1-16, Feb 1995.

[22] E. Rocon, J. M. Belda-Lois, A. F. Ruiz, M. Manto, J. C. Moreno, and J. L. Pons, "Design and validation of a rehabilitation robotic exoskeleton for tremor assessment and suppression," (in English), Ieee Transactions on Neural Systems and Rehabilitation Engineering, Article; Proceedings Paper vol. 15, no. 3, pp. 367-378, Sep 2007.

[23] E. Rocon, J. Á. Gallego, J. M. Belda-Lois, J. Benito-León, and J. L. Pons, "Biomechanical Loading as an Alternative Treatment for Tremor: A Review of Two Approaches," Tremor other Hyperkinet Mov (N Y), vol. 2, 2012. 
[24] S. Dosen et al., "Online Tremor Suppression Using Electromyography and Low-Level Electrical Stimulation," (in English), Ieee Transactions on Neural Systems and Rehabilitation Engineering, Article vol. 23, no. 3, pp. 385-395, May 2015.

[25] J. A. Gallego, E. Rocon, J. M. Belda-Lois, and J. L. Pons, "A neuroprosthesis for tremor management through the control of muscle co-contraction," (in English), Journal of Neuroengineering and Rehabilitation, Article vol. 10, p. 12, Apr 2013, Art. no. 36.

[26] C. T. Freeman, P. Sampson, J. H. Burridge, and A. M. Hughes, "Repetitive control of functional electrical stimulation for induced tremor suppression," (in English), Mechatronics, Article vol. 32, pp. 79-87, Dec 2015.

[27] L. P. Maneski et al., "Electrical stimulation for the suppression of pathological tremor," (in English), Medical \& Biological Engineering \& Computing, Article vol. 49, no. 10, pp. 1187-1193, Oct 2011.

[28] A. Prochazka, J. Elek, and M. Javidan, "ATTENUATION OF PATHOLOGICAL TREMORS BY FUNCTIONAL ELECTRICAL-STIMULATION .1. METHOD," (in English), Annals of Biomedical Engineering, Article vol. 20, no. 2, pp. 205-224, 1992.

[29] A. P. L. Bo, C. Azevedo-Coste, C. Geny, P. Poignet, and C. Fattal, "On the Use of FixedIntensity Functional Electrical Stimulation for Attenuating Essential Tremor," (in English), Artificial Organs, Editorial Material vol. 38, no. 11, pp. 984-991, Nov 2014.

[30] N. C. Townsend and R. A. Shenoi, "Gyrostabilizer Vehicular Technology," (in English), Applied Mechanics Reviews, Review vol. 64, no. 1, p. 14, Jan 2011, Art. no. 010801.

[31] N. C. Townsend and R. A. Shenoi, "Control Strategies for Marine Gyrostabilizers," (in English), Ieee Journal of Oceanic Engineering, Article vol. 39, no. 2, pp. 243-255, Apr 2014.

[32] W. D. Hall, "Hand-held gyroscopic device," Patent US 5058571 A, 1991.

[33] M. A. Kalvert, "Adjustable and tunable hand tremor stabilizer," Patent US 6730049 B2, 2004.

[34] W. Palm, System Dynamics, 3rd ed. New York, NY: McGraw Hill, 2014.

[35] P. de Leva, "Adjustments to Zatsiorsky-Seluyanov's segment inertia parameters," (in English), Journal of Biomechanics, Article vol. 29, no. 9, pp. 1223-1230, Sep 1996.

[36] W. B. Drake and S. K. Charles, "Passive Stiffness of Coupled Wrist and Forearm Rotations," (in English), Annals of Biomedical Engineering, vol. 42, no. 9, pp. 1853-1866, 2014.

[37] A. L. Pando, H. Lee, W. B. Drake, N. Hogan, and S. K. Charles, "Position-Dependent Characterization of Passive Wrist Stiffness," IEEE Transactions on Biomedical Engineering, vol. 61, no. 8, pp. 2235-2244, Aug 2014.

[38] D. Formica, S. K. Charles, L. Zollo, E. Guglielmelli, N. Hogan, and H. I. Krebs, "The Passive Stiffness of the Wrist and Forearm," Journal of Neurophysiology, vol. 108, pp. 1158-1166, 2012. 
[39] E. J. Perreault, R. F. Kirsch, and P. E. Crago, "Multijoint dynamics and postural stability of the human arm," (in English), Experimental Brain Research, Article vol. 157, no. 4, pp. 507-517, Aug 2004.

[40] T. Sinkjaer and R. Hayashi, "REGULATION OF WRIST STIFFNESS BY THE STRETCH REFLEX," (in English), Journal of Biomechanics, Article vol. 22, no. 11-12, pp. 1133-1140, 1989.

[41] M. Halaki, N. O'Dwyer, and I. Cathers, "Systematic nonlinear relations between displacement amplitude and joint mechanics at the human wrist," (in English), Journal of Biomechanics, Article vol. 39, no. 12, pp. 2171-2182, 2006.

[42] G. Deuschl, P. Bain, M. Brin, and C. Ad Hoc Sci, "Consensus statement of the Movement Disorder Society on tremor," (in English), Movement Disorders, Article; Proceedings Paper vol. 13, pp. 2-23, 1998.

[43] C. C. R. Elble, S. Fahn, M. Hallett, J. Jankovic, J. Juncos, et al., "The essential tremor rating assessment scale (TETRAS)," Movement Disorders, vol. 23, pp. S357-S357, 2008.

[44] H. Higashiyama, M. Yamada, Y. Kazao, and M. Namiki, "Characteristics of active vibration control system using gyro-stabilizer," (in English), Engineering Structures, Article vol. 20, no. 3, pp. 176-183, Mar 1998.

[45] H. Kanki, Y. Nekomoto, H. Monobe, H. Ogura, and K. Kobayashi, "DEVELOPMENT OF CMG ACTIVE VIBRATION CONTROL DEVICE FOR GONDOLA," (in English), Jsme International Journal Series C-Dynamics Control Robotics Design and Manufacturing, Article vol. 37, no. 3, pp. 468-470, Sep 1994.

[46] G. Herrnstadt, "On-Off Tremor Suppression Orthosis with Electromagnetic Brake," International Journal of Mechanical Engineering and Mechatronics, vol. 1, no. 2, pp. 7$14,2013$.

[47] C. Weller. (2016). This glove could help Parkinson's patients stop shaking.

[48] "Liquid body armor," (in English), American Ceramic Society Bulletin, News Item vol. 86, no. 3, pp. A15-A15, Mar 2007.

[49] S. B. Godfrey, R. J. Holley, and P. S. Lum, "Evaluation of HEXORR Tone Assistance Mode Against Spring Assistance," (in English), Ieee Transactions on Neural Systems and Rehabilitation Engineering, Article vol. 23, no. 4, pp. 610-617, Jul 2015.

[50] D. Mozaffarian et al., "Heart Disease and Stroke Statistics-2015 Update A Report From the American Heart Association," (in English), Circulation, Article vol. 131, no. 4, pp. E29-E322, Jan 2015.

[51] H. F. M. Van Der Loos, "Rehabilitation and Health Care Robotics," Springer Handbook of Robotics, pp. 1223-1251, 2008.

[52] P. Maciejasz, J. Eschweiler, K. Gerlach-Hahn, A. Jansen-Troy, and S. Leonhardt, "A survey on robotic devices for upper limb rehabilitation," (in English), Journal of Neuroengineering and Rehabilitation, Review vol. 11, p. 29, Jan 2014, Art. no. 3. 
[53] J. Stein, "Robotics in rehabilitation: technology as destiny," American journal of physical medicine \& rehabilitation, vol. 91, no. 11 Suppl 3, pp. S199-203, 2012.

[54] P. S. Lum, C. G. Burgar, P. C. Shor, M. Majmundar, and M. Van der Loos, "Robot-assisted movement training compared with conventional therapy techniques for the rehabilitation of upper-limb motor function after stroke," (in English), Archives of Physical Medicine and Rehabilitation, Article; Proceedings Paper vol. 83, no. 7, pp. 952-959, Jul 2002.

[55] S. Hesse, G. Schulte-Tigges, M. Konrad, A. Bardeleben, and C. Werner, "Robot-assisted arm trainer for the passive and active practice of bilateral forearm and wrist movements in hemiparetic subjects," (in English), Archives of Physical Medicine and Rehabilitation, Article vol. 84, no. 6, pp. 915-920, Jun 2003.

[56] D. Leonardis et al., "An EMG-Controlled Robotic Hand Exoskeleton for Bilateral Rehabilitation," (in English), Ieee Transactions on Haptics, Article vol. 8, no. 2, pp. 140151, Apr-Jun 2015.

[57] H. I. Krebs, "Robot-aided neurorehabilitation," IEEE transactions on rehabilitation engineering : a publication of the IEEE Engineering in Medicine and Biology Society, vol. 6, no. 1, 1998.

[58] C. G. Burgar, P. S. Lum, P. C. Shor, and H. F. M. Van der Loos, "Development of robots for rehabilitation therapy: The Palo Alto VA/Stanford experience," (in English), Journal of Rehabilitation Research and Development, Article vol. 37, no. 6, pp. 663-673, Nov-Dec 2000.

[59] R. J. Sanchez et al., "Automating arm movement training following severe stroke: Functional exercises with quantitative feedback in a gravity-reduced environment," (in English), Ieee Transactions on Neural Systems and Rehabilitation Engineering, Article vol. 14, no. 3, pp. 378-389, Sep 2006.

[60] S. Hesse, C. Werner, M. Pohl, S. Rueckriem, J. Mehrholz, and M. L. Lingnau, "Computerized arm training improves the motor control of the severely affected arm after stroke - A single-blinded randomized trial in two centers," (in English), Stroke, Article vol. 36, no. 9, pp. 1960-1966, Sep 2005.

[61] M. Kuttuva et al., "The Rutgers Arm, a rehabilitation system in virtual reality: A pilot study," (in English), Cyberpsychology \& Behavior, Article; Proceedings Paper vol. 9, no. 2, pp. 148-151, Apr 2006.

[62] S. Masiero, A. Celia, G. Rosati, and M. Armani, "Robotic-assisted rehabilitation of the upper limb after acute stroke," (in English), Archives of Physical Medicine and Rehabilitation, Article vol. 88, no. 2, pp. 142-149, Feb 2007.

[63] J. Iqbal, "A portable rehabilitation device for the Hand," presented at the Annual International Conference of the IEEE Engineering in Medicine and Biology, 2010.

[64] D. J. Reinkensmeyer, C. T. Pang, J. A. Nessler, and C. C. Painter, "Web-based telerehabilitation for the upper extremity after stroke," (in English), Ieee Transactions on Neural Systems and Rehabilitation Engineering, Article vol. 10, no. 2, pp. 102-108, Jun 2002, Art. no. Pii s 1534-4320(02)05944-2. 
[65] M. J. Johnson, H. F. M. Van der Loos, C. G. Burgar, P. Shor, and L. J. Leifer, "Experimental results using force-feedback cueing in robot-assisted stroke therapy," (in English), Ieee Transactions on Neural Systems and Rehabilitation Engineering, Article vol. 13 , no. 3 , pp. 335-348, Sep 2005.

[66] H. Sugarman, E. Dayan, A. Weisel-Eichler, and J. Tiran, "The Jerusalem TeleRehabilitation System, a new low-cost, haptic rehabilitation approach," (in English), Cyberpsychology \& Behavior, Article; Proceedings Paper vol. 9, no. 2, pp. 178-182, Apr 2006.

[67] G. Kwakkel, B. J. Kollen, and H. I. Krebs, "Effects of robot-assisted therapy on upper limb recovery after stroke: A systematic review," (in English), Neurorehabilitation and Neural Repair, Review vol. 22, no. 2, pp. 111-121, Mar-Apr 2008.

[68] H. Baruh, Applied Dynamics. 2015.

[69] M. Kim. (2016). Euler-Lagrange tool package (1.5 ed.). Available: https://www.mathworks.com/matlabcentral/fileexchange/49796-euler-lagrange-toolpackage 


\section{APPENDIX A. $\quad$ LITERATURE REVIEW}

\section{A.1 Tremor Suppression}

\section{A.1.1 Background}

Tremor is a rhythmical, involuntary oscillatory movement of a body part produced by reciprocally innervated antagonist muscles [46]. There are three primary categories of tremor: resting tremor, postural tremor, and kinetic tremor. Resting tremor arises in a body part that is relaxed and completely supported against gravity, postural tremor occurs when the body is voluntarily maintained against gravity, while kinetic tremor is apparent during voluntary movement [46]. Tremor is generally manifested between 3 to $12 \mathrm{~Hz}$, while the frequency of ADLs is typically under $2 \mathrm{~Hz}$ [46]. Unfortunately, the current treatments for tremor are medication, which may have unwanted side effects, and neurosurgery [46]. Neither of these treatments have guaranteed success. In fact, about 40 to $50 \%$ of patients do not find relief from the current treatments [46]. Additionally, tremor generally gets worse with time, so one treatment may work for a time but not permanently [46].

\section{A.1.2 Alternative Methods for Tremor Suppression}

We investigated five alternative methods that can potentially be used for tremor suppression. These methods are: gyroscopes, magnetorheological fluid (MRF), liquid armor, electromagnetic brakes, and a mechanical low-pass filter. 
Gyroscopes: Gyroscopes resist a change in angular momentum and can be used to resist movements caused by tremor. The gyroscope can be made to spin faster or to have a larger inertia to increase its resistance to tremor. A gyroscope can be easily adaptable to different sized limbs. Variable speed gyroscopes have not been used to optimize its tremor suppression based on the current size of the tremor.

We found two patents for a gyroscopic tremor suppression device with one pending. A patent is pending for the Gyroglove. The Gyroglove is a single flywheel gyroscope that is located on the hand. It is powered by a battery and has a tiny integrated controller that drives a precession hinge and turntable, and a responsive gyroscope [47]. The Gyroglove is expected to sell for about $\$ 700$. The Gyroglove is light, wearable, can eliminate up to $90 \%$ of tremor, and provides free motion that is just slower [47]. However, the Gyroglove is loud and has a constant speed gyroscope that cannot adapt to different sized people or to tremor fluctuations. One of the patents is of a wearable splint that can mount one or more gyroscopes on it. Each of these gyroscopes can be positioned to optimize their benefit [33]. The gyroscopes have two-flywheels to counteract more types of tremor, but the splint is not portable and is bulky. The other patent is of a single flywheel gyroscope that is attached to the hand [32]. It attaches batteries to the flywheel to add to the gyroscopic effect. It is similar to the Gyroglove but less sophisticated in design.

MRF: MRF consist of a suspension of microscopic magnetizable particles in a nonmagnetic carrier medium, usually water or some type of synthetic oil [18]. Without a magnetic field the fluid behaves in a Newtonian manner. When a magnetic field is applied the microscopic particles suspended in the fluid become oriented and form chains along the magnetic flux lines, changing the fluid's rheology. The presence of the particle chains causes the fluid to exhibit a yield stress if flow occurs perpendicular to the magnetic flux lines. The fluid then behaves like a 
Bingham plastic. Interestingly, the observed yield stress is related to the applied magnetic field, which makes MRF ideal to use as a low-power tunable damper. MRF actuators have a fast response time, high-fidelity control, can be customizable to the patient, and can be reactive to various tremor magnitudes, however they are large and would need to be located at each individual joint. Currently MRF is being used to create variable dampers that can be used to dampen out tremor at each joint $[18,19]$. In another application, MRF is used to implement variable dampening in a viscous beam [17].

Liquid Armor: Liquid armor is a polyethylene glycol liquid with tiny hard particles of silica suspended in it [48]. This creates a thick syrupy fluid in which Kevlar is soaked. The fabric then feels oily to the touch. When the fabric is struck by a knife or bullet the fabric immediately becomes rigid. When you stop the stress, it goes back to being a liquid. Liquid armor is a shear thickening fluid and currently has no application in tremor suppression. It is unlikely that liquid armor can be applied to tremor suppression.

Electromagnetic brakes: There is currently a device that uses electromagnetic brakes to stop tremor in the elbow [46]. The device needs to measure the tremor and then activate the brake when the tremor is at its peak amplitude to stop it effectively [46]. The tremor oscillates and the goal is to turn the brake on at a certain point of each oscillation and then off again. The goal is to try and minimize the time that the brake is on. It is challenging to properly time the brake and it creates a choppy motion to the user since the brake counters all movement. This causes the brake to impede the user's normal movement when the brake is activated. Overall, it is concluded that electromagnetic brakes do not have a great application to tremor suppression.

Low-pass Filter: Since a body has mass, stiffness, and damping it acts as a low-pass filter. Tremor suppression can be obtained at a joint by modifying the mass, stiffness, and damping at 
that particular joint to change the cutoff frequency. Since normal movement generally occurs at a frequency under $2 \mathrm{~Hz}$ an ideal cutoff frequency would be at $2 \mathrm{~Hz}$. If the low-pass characteristics of the joint can be modified to have a cutoff frequency of $2 \mathrm{~Hz}$ then it would let normal movement occur unimpeded while blocking higher frequency movements, such as from a tremor. The downside is that a low-pass filter would need to be applied to each joint and it would need to be personalized to each individual. Another complication is that joint stiffness and damping changes with muscle activation. Currently there is a wrist orthosis in which the actuator is a viscous beam that applies passive velocity proportional resistance to suppress tremor [14].

\section{A.2 Rehabilitation Robotics}

There is potentially some overlap between tremor suppression devices and rehabilitation robotics. Since rehabilitation robotics are far more developed than tremor suppression devices, it may be possible to take advantage of advances in rehabilitation robotics in the development of tremor suppression devices. Therefore, a general review of rehabilitation robotics is included here.

\section{A.2.1 Background}

There are 15 million strokes each year worldwide with about 5 million resulting in permanent disability [49]. In the United States alone there are nearly 800,000 strokes a year with 600,000 being the individuals first stroke [50]. Additionally, from 2001 to 2011 the rate of death after a stroke fell by $35.1 \%$ resulting in an increased number of stroke survivors [50]. Though between 50 and $70 \%$ of stroke survivors regain functional independence, nearly $80 \%$ of survivors suffer from upper extremity hemiparesis [49]. Hemiparesis is a weakness on one side of the body [51]. Another common impairment due to stroke is abnormal tone, which causes an increase in felt 
resistance to passive movement of a limb [51]. Limited hand function and range of motion (ROM) often occurs post-stroke due to spasticity, hypertonia, or muscle weakness [49].

There are four main types of rehabilitation robots: (1) therapy robots, (2) robots that support activities of daily living (ADLs), such as a device for tremor suppression that allows a user to perform ADLs, (3) assistive robots, and (4) prosthetics or functional neural stimulation or technology for diagnosis and monitoring of patients during ADLs [51]. Therapy robots aim to retrain the movement or manipulation abilities of disabled individuals. The concept behind therapy robots is that the human neuromuscular system has use-dependent plasticity, ie. use alters the properties of the neurons and muscles, which includes the pattern of connectivity and thus function [51]. The original therapy robots, such as the MIT-Manus and the MIME focused on the proximal joints of the arm [49]. Modern therapy robots now focus more on distal joints because they have a greater impact on functional use [49]. There are many therapy robots that focus on just one DOF, while others target multiple DOF of the arm [52]. Assistive robots focus on manipulation, mobility, or cognition and include wheelchair mounted robots or autonomous robots.

We were unable to find any wearable devices that actively counteract the spasticity or tone of the hand. The main difficulty is that it is hard to make a device that does not impede the use of the hand, is not so large that no one would want to use it, or that has a low enough cost. Depending on the severity of the patient however, any improvement may be considered acceptable. If that is the case then one potential option is a single DOF device that assists in opening and closing the hand. This single DOF device would allow for the patient to grasp things but would not restore functional use of the hand.

Studies have found that increased repetitions and repetitive, task-specific movements are effective at rehabilitating the upper extremity after stroke [49]. Since robotics allow for high 
repetitions of task-specific movements the area of therapy robotics has been greatly expanding. There are currently over 120 different therapy robots that have been investigated [52]. Almost all physical therapy robots focus on treating stroke or spinal cord injury due to there being many people, a high cost of rehabilitation, and because of the repetitive nature of rehabilitation [51]. Almost all of the current devices are designed to be used in rehab centers or clinics; very few are marketed toward being used at home. This is due to the high cost of these devices and the belief that the rehab process needs to be closely monitored. Currently, there is an increasing demand for home-based therapy robots that are easy to use, intuitive, fast to set up (under 5 minutes), and a reasonable price [52]. To allow for supervision the current home-use devices have looked into telerehab to send reports of the results to a therapist [51-53]. Additionally, this increased demand for home based robots has led to some therapy robots having a complex form for clinical use and a simpler form for home use [52].

\section{A.2.2 Grand Challenges: How to Optimize Use-Dependent Plasticity}

The grand challenge in therapy robotics is determining what the robot should do in cooperation with the patient's own movements in order to maximize improved mobility [51]. A key problem is to determine appropriate movement tasks, such as what movement should the patient practice and what feedback should be given [51]. Another key problem is determining the appropriate pattern of mechanical input to the patient during the movement tasks. This includes what forces the robot should apply to the limbs to provoke plasticity [51]. Unfortunately, there are 2 main roadblocks in developing an effective therapy robot [51]. The first is we do not know what the therapy robot should do. The optimal movement tasks and mechanical inputs are still unknown. The second is technological. The robot needs to be light, with a high degree of freedom (DOF), wearable, and have a high bandwidth. The robot requires a high bandwidth so that it can impose 
the desired movement when the patient is paralyzed and then fade to nothing as the patient recovers [51].

\section{A.2.3 Types of Assistance, Mechanical Designs, and Actuation}

Devices for upper limb rehabilitation may provide different types of motion assistance: passive, active, haptic, and coaching [52]. Passive devices are unable to move limbs, but may resist movement. A patient must be able to move their limb for this device to be useful. Passive devices are generally less complex, safer (they generally only have brakes), cheaper, and lighter than other types of device [52]. Active devices have active actuators and can be used to help those who are unable to move their limbs [52]. Haptic devices interface with the user through touch. Haptic devices often provide a resistive force or vibration and are often used with a virtual environment [52]. A coaching device neither assists nor resist movement, it just tracks movement and gives feedback of the performance [52].

The mechanical design of a therapy robot is either end-effector based or exoskeleton based. An end-effector based device contacts the subject's limbs at the most distal part of the device, which simplifies the structure but may complicate the control [52]. An exoskeleton based device needs to adjust the lengths of particular segments of the manipulator to the lengths of the segments of the patient's arm, which can take a while to set up. Additionally, the position of the center of rotation of many joints of the body change a lot during rotation, such as with the shoulder [52]. This requires the device to have a special mechanism to ensure safety and comfort. End-effector based devices have a more complicated structure but are easier to control.

Energy to the actuators on a therapy robot is generally provided by electric current, hydraulic fluid, or pneumatic pressure [52]. The selection of the energy source determines the 
types of actuators that can be used in the system. Typical actuators in upper limb rehabilitation are electric actuators, hydraulic actuators, pneumatic actuators, series elastic actuators, and functional electrical stimulation (FES) [52]. Electric actuators are the most common used. Hydraulic actuators provide high forces but are heavy and complicated. Pneumatic actuators have a lower impedance and weigh less than electric actuators but are very nonlinear and require special compressors or containers of compressed air [52]. Series elastic actuators lower the inertia and impedance of electric actuators creating a more accurate and stable force control and it is safer. Lastly, FES uses a current to activate nerves and contract innervated muscles but is difficult to achieve precise movement and can be painful [52].

\section{A.2.4 Methods to Induce Plasticity and to Improve a Patient's Health}

There have been numerous studies that have searched for optimal therapy strategies to induce plasticity to improve a patient's health. Studies have found that monotonous exercises lead to worse retention; therefore, adaptive therapy is better than a fixed pattern [52]. It was also found that repeated practice of skilled movements plays a key role in stimulating plasticity [53]. In fact, repetitive movements are more effective than treatment from a therapist focused on teaching techniques or encouraging self-practice [54]. Unassisted repetitive motion is best when the patient

can do a portion of the movements [54]. Another potential way to induce plasticity is via hebbian learning. Hebbian learning states that if a user is trying to move and is generating torque as the movement occurs (providing positive work) then there is synchronization between effort and movement. This in theory has the sensory feedback arrive at the cortex as the pathway is activated leading to an improved rehabilitation [49]. 
Positive outcomes are influenced by many factors, such as the health of the patient, the attention and effort that the patient gives during the exercise, the duration and intensity of the treatment, and the tasks that are performed are some examples [52]. For optimal results, maximal voluntary contraction (muscle strength) is not as important as properly timed activity of agonist and antagonist muscles (coordination of movement) [52]. Exercises are more effective when they maintain the attention of the patient and if they invoke implicit learning (learning without awareness) in the patient [52]. Many functional gains are more dependent on wrist and hand movement than mobility of the shoulder or elbow [52]. Therefore, when functional goals are the main focus, emphasis should be placed on wrist and hand exercises. For the most success, early, intensive, and task specific approaches are the best [55]. The goal of rehab is to induce plasticity by maximizing the number of repetitions, the patient's attention, and the patient's effort [52].

Assistive strategies are impedance based, counter-balanced based, EMG-based, or performance based [52]. Impedance based strategies have the patient follow a path and the device intervenes when the patient leaves the path by providing a restoring force that increases with deviation from the desired trajectory. Counter-balanced based strategies provide a partial, passive, or active weight counterbalance to the limb making the exercise easier by lowering the force needed to move against gravity. EMG-based strategies use sEMG signals to trigger a proportionally controlled assistance that encourages effort. Lastly, a performance based strategy monitors the performance and adapts some aspects of help (force, time, path, stiffness) based on the current and past performance.

Challenge-based strategies are either error amplifying or constraint-induced. Error amplifying strategies are based on the theory that faster improvement is achieved when the error is amplified. It tracks the deviations and either increases the observed kinematic error or 
amplifies the error seen on a screen [52]. Constraint induced strategies promote use of the affected limb by constraining the non-affected limb $[52,54]$. Intensive repetitive exercise of the more affected limb leads to passive cortical reorganization in the motor cortex [54]. Challengebased strategies are the most effective in mildly impaired subjects [55].

\section{A.2.5 Current Devices}

Some examples of therapy robots are listed in this section.

An EMG controlled robotic hand exoskeleton for bilateral rehabilitation is discussed in [56]. The device was designed to leave the patients palm and finger tips free.

MIT-Manus: A planar 2-joint arm robot that is designed to exercise the shoulder and elbow of the patient. It is the simplest mechanical design that allows planar movement while allowing a large range of forces without requiring force feedback [57].

MIME: Uses a puma-560 robot arm and a splint to attach the patient's arm to the robot. The robot has $6 \mathrm{DOF}$, which leads to natural movement of the limb. It incorporates a mirror-image mode, which measures the movement of the less impaired limb and controls the impaired limb to follow this motion. Additionally, the robot has an active-constrained mode that resists movement away from the target but does not assist going toward the target. The bimanual mode uses mirrorimage movements to help the affected limb stay in a mirror image position of the good arm. Recovery from hemiplegia is mediated by corticospinal ipsilateral pathways and they appear to be active during bilateral movement $[54,58]$.

T-WREX: A passive exoskeleton that supports the arm against gravity using elastic bands, while still allowing a large range of motion of the arm [59]. 
Bi-Manu-Track: Uses a motor for each hand to allow bimanual wrist flexion and extension movements. Tilting the handles downward allows for forearm pronation and supination movements [60].

Rutgers hand: Uses pneumatic cylinders to help extend and flex the fingers [61].

NeReBot: Uses wires to slowly move the patient's arms in spiral paths. Three independently driven wires connect to the arm with a splint and the wire length then changes to move the arm [62].

HEXORR: A robot that exercises the wrist and fingers. HEXORR treats the fingers as 1 DOF. The device was designed to treat the spasticity, hypertonia, or muscle weakness of the hand. HEXORR has a linear spring mode and a hypertonia mode. During the linear spring mode, the assistance is increased as distance from the target increases. The hypertonia mode counteracts hypertonia by providing a roughly linear assistance profile that increases with extension. The motors provide compensation for gravity and friction. The device only assists for extension of the fingers [49].

HEXOSYS: A direct driven, 4 DOF, under actuated portable hand robot. The design uses optimization techniques to size each link [63].

\section{A.2.6 Low-Cost Rehabilitation Robots}

There are four ways to lower the cost of a rehabilitation robot: (1) to use a planar robot, (2) to make it reconfigurable, (3) to take advantage of modularity, and (4) to be specific [52]. A planar robot is usually an end-effecter based robot and the device moves in a plane but the patient's joints can still move in 3D space [52]. Modularity uses optional parts that may adapt the device to a 
specific condition or to perform additional exercises [52]. A reconfigurable robot allows the mechanical structure to be modified to adapt to different training exercises or conditions without adding additional parts [52]. For instance, a planar robot can be made to change between working in the horizontal and vertical planes to increase the range of available movements. This allows for one motor or sensor to be used to control different types of movements. Lastly, to lower the cost of a rehab robot it needs to be specific in what it does. The more features added to the robot will cause its price to increase. The device should be designed to treat a specific condition, a specific severity level, and a specific limb or nearby limbs. For instance, the wrist and fingers go well together. A potential idea is to make multiple devices that can be purchased individually. For example, if someone has predominantly a wrist issue they can simply purchase the wrist robot. If they also have a shoulder injury, then they can purchase a shoulder robot as well.

One current low-cost robot uses a force feedback joystick with telerehabilitation ability [64]. Another uses a force feedback steering wheel that restrains the less impaired arm so that the patient has to use the impaired limb [65]. There is also a telerehabilitation system that allows for low-cost communication between a therapist and the patient at home [66].

\section{A.2.7 Conclusion}

A rehabilitation robot must be safe, intuitive, fast to set up, a reasonable price, comfortable, easy to use, and designed with low intrinsic impedance $[52,53]$. The motors need to be back drivable to provide safety measures [52]. The patient should perform several hundred repetitions per session because that many were required to promote cortical plasticity in animal models [49]. The development of low-cost rehab robots will be aided by making simpler robots that are more targeted. For instance, instead of making a robot that can treat various neurological conditions and 
at different levels of severity, the device can be made for specific issues, such as a mildly affected stroke patient. For instance, a robot for less affected individuals will be more resistive or actively constrained (resists when moving away from the target), while a robot for more severely affected individual will be more assistive. A potential idea would be to develop a reconfigurable endeffector device to exercise the shoulder and the elbow with planar motions. Then an exoskeleton device can be designed to exercise the wrist and forearm and potentially the fingers as well.

Further research should investigate the efficacy of hebbian learning versus alternative strategies. Additionally, future research should seek to better understand current techniques used by physical therapists and to implement these techniques in future robots. In the future, the efficacy of rehabilitation robots needs to be improved. The MIT-Manus, MIME, ARM Guide, Inmotion shoulder-elbow robot, and the Bi-Manu track have been tested in at least one random clinical trial as of 2008 [67]. Most clinical studies used chronic stroke patients and they had only modest improvements of 3-6 points using the upper extremity fugl-meyer scale. Very few other devices have been clinically tested, which limits our ability to judge the efficacy of these rehabilitation devices. Due to there being few thorough clinical studies it is unknown if the plasticity of the human nervous system cannot sufficiently reduce disability. There is possibly a limited ability of the human brain to repair itself and the devices are not the problem [53]. It is noted however that acute stroke patients may recover better than chronic stroke patients because early and high intensity rehab should lead to higher improvement [54]. 


\section{APPENDIX B. \\ DERIVATION OF STATE SPACE EQUATIONS OF MOTION}

\section{B.1 Equations of Motion}

Our system included three rigid bodies: hand, motor, and flywheel. To develop the nonlinear equations of motion governing the dynamics of this system, we used Lagrange's method [68]. More specifically, we defined coordinate frames for each of the three bodies and established the kinematic relationships between these frames, calculated the Lagrangian for each body, and derived the final EOM.

\section{B.1.1 Coordinate Frames and Kinematic Relationships}

Four frames define the position and orientation of all rigid bodies (Figure 2-1). The inertial frame, $\boldsymbol{X}_{0} \boldsymbol{Y}_{0} \boldsymbol{Z}_{0}$, has its origin in the wrist joint center and does not move. Gravity acts along $\boldsymbol{Y}_{0}$. The body-fixed frame of the hand, $\boldsymbol{x}_{1} \boldsymbol{y}_{1} \boldsymbol{z}_{1}$, which has its origin in the wrist joint center, defines the hand, with $\boldsymbol{x}_{1}, \boldsymbol{y}_{1}$, and $\boldsymbol{z}_{1}$ pointing from proximal to distal, volar to dorsal, and lateral to medial, respectively. Frame $\boldsymbol{X Y Z}$ orients the structure holding the gyroscope relative to the hand, with $\boldsymbol{Z}$ pointing along the precession axis. Finally, the body-fixed frame of the gyroscope (flywheel + motor), $\boldsymbol{x y z}$, has its origin in the center of the precession axis, with $\boldsymbol{y}$ along the spin axis.

Rotation matrices were used to transform from one frame to another:

$$
\begin{aligned}
& X_{0} Y_{0} Z_{0} \stackrel{R_{1}}{\rightarrow} x_{1} y_{1} z_{1} \stackrel{R_{2}}{\rightarrow} X Y Z \stackrel{R_{3}}{\rightarrow} x y z \\
& \boldsymbol{X}_{0} \boldsymbol{Y}_{0} \boldsymbol{Z}_{0} \stackrel{R_{1}^{T}}{\leftarrow} \mathbf{x}_{1} \mathbf{y}_{1} \mathbf{z}_{1} \stackrel{R_{2}^{T}}{\leftarrow} \mathrm{XYZ} \stackrel{R_{3}^{T}}{\leftarrow} \mathrm{xyz}
\end{aligned}
$$


Rotation matrix $R_{1}$, which describes the orientation of the hand relative to the inertial frame, depends on the DOF at the wrist joint (FE, RUD, or PS), as shown in Table B-1. In other words, $R_{1}$ defines the wrist joint axis as either $\boldsymbol{Z}_{0}=\boldsymbol{z}_{1}$ (FE), $\boldsymbol{Y}_{0}=\boldsymbol{y}_{1}$ (RUD), or $\boldsymbol{X}_{0}=\boldsymbol{x}_{1}$ (PS). The wrist joint angle is always $\theta$.

Table B-1: Rotation Matrix and Angular Velocity of Frame $\boldsymbol{x}_{1} \boldsymbol{y}_{1} \boldsymbol{z}_{1}$ for Each Wrist Joint Axis

\begin{tabular}{|c|c|c|c|}
\hline Flexion-Extension (FE) & Radial-UInar Deviation (RUD) & Pronation-Supination (PS) \\
\hline$R_{1}=\left[\begin{array}{ccc}\cos \theta & \sin \theta & 0 \\
-\sin \theta & \cos \theta & 0 \\
0 & 0 & 1\end{array}\right]$ & $R_{1}=\left[\begin{array}{ccc}\cos \theta & 0 & -\sin \theta \\
0 & 1 & 0 \\
\sin \theta & 0 & \cos \theta\end{array}\right]$ & $R_{1}=\left[\begin{array}{ccc}1 & 0 & 0 \\
0 & \cos \theta & \sin \theta \\
0 & -\sin \theta & \cos \theta\end{array}\right]$ \\
\hline$\omega_{x_{1} y_{1} z_{1}}=\left[\begin{array}{lll}0 & 0 & \dot{\theta}\end{array}\right]^{T}$ & $\omega_{x_{1} y_{1} z_{1}}=\left[\begin{array}{lll}0 & \dot{\theta} & 0\end{array}\right]^{T}$ & $\omega_{x_{1} y_{1} z_{1}}=\left[\begin{array}{lll}\dot{\theta} & 0 & 0\end{array}\right]^{T}$ \\
\hline
\end{tabular}

The second rotation matrix, $R_{2}$, orients the gyroscope on the hand by transforming $\boldsymbol{x}_{1} \boldsymbol{y}_{1} \boldsymbol{z}_{1}$ into $\boldsymbol{X Y Z}$ as follows: $\boldsymbol{x}_{1} \boldsymbol{y}_{1} \boldsymbol{z}_{1}$ is rotated first about $\boldsymbol{y}_{1}$ by $\beta_{1}$, then about $\boldsymbol{z}_{\mathbf{1}}{ }^{\prime}$ by $\beta_{2}$, and lastly about $\boldsymbol{y}_{1}{ }^{\prime \prime}$ by $\beta_{3}$. Therefore,

$$
\text { with } R_{2}=\left[\begin{array}{l}
\boldsymbol{X} \\
\boldsymbol{Y} \\
\boldsymbol{Z}
\end{array}\right]=R_{2}\left[\begin{array}{l}
\boldsymbol{x}_{1} \\
\boldsymbol{y}_{1} \\
\boldsymbol{z}_{1}
\end{array}\right],
$$

In other words, orientation constants $\beta_{1}, \beta_{2}$, and $\beta_{3}$ fully define the orientation of the precession axis relative to the hand. Likewise, these orientation constants define the orientation of the spin axis relative to the precession axis (and the hand) when the gyroscope is in its initial position $(\emptyset=0)$. As mentioned above, the precession axis can take on three cardinal orientations relative to the wrist axis, and the spin axis can take on two cardinal orientations relative to the precession axis (ignoring the trivial case in which the spin axis is parallel to the precession axis), 
resulting in six cardinal gyroscope orientations for each of the three wrist joint axes (Figure 2-2). The orientation constants define these six cardinal gyroscope orientations (Table B-2).

Table B-2: The Orientation Constants to Obtain the 6 Cardinal Orientations of a Gyroscope Relative to the Hand

\begin{tabular}{|c|c|c|c|c|c|c|}
\hline & O1 & O2 & O3 & O4 & O5 & 06 \\
\hline$\beta_{1}$ & $0^{\circ}$ & $90^{\circ}$ & $0^{\circ}$ & $0^{\circ}$ & $90^{\circ}$ & $90^{\circ}$ \\
\hline$\beta_{2}$ & $0^{\circ}$ & $0^{\circ}$ & $-90^{\circ}$ & $-90^{\circ}$ & $90^{\circ}$ & $90^{\circ}$ \\
\hline$\beta_{3}$ & $0^{\circ}$ & $0^{\circ}$ & $0^{\circ}$ & $-90^{\circ}$ & $90^{\circ}$ & $0^{\circ}$ \\
\hline
\end{tabular}

The third rotation matrix, $R_{3}$, transforms $\boldsymbol{X Y Z}$ into the gyroscope body-fixed frame, $\boldsymbol{x y z}$, which precesses with the gyroscope.

$$
R_{3}=\left[\begin{array}{ccc}
\cos \emptyset & \sin \emptyset & 0 \\
-\sin \emptyset & \cos \emptyset & 0 \\
0 & 0 & 1
\end{array}\right]
$$

Matrices $R_{1}$ and $R_{2}$ were used to facilitate developing the equations of motion for each of the 18 cardinal configurations (Figure 2-3). Developing these equations of motion following Lagrange's method requires calculation of the Lagrangian for each rigid body. The Lagrangian is the difference between the kinetic and potential energy of a rigid body and depends on the position of the COM and total angular velocity of the rigid body.

\section{B.1.2 Lagrangian for Each Rigid Body}

Body 1. Hand: The position of the COM and angular velocity of the body-fixed frame of the hand, expressed in $\boldsymbol{x}_{1} \boldsymbol{y}_{1} \boldsymbol{z}_{1}$, are

$$
\begin{gathered}
\boldsymbol{r}_{w}=\left[\begin{array}{lll}
x_{w} & y_{w} & z_{w}
\end{array}\right]^{T} \\
\boldsymbol{\omega}_{w}=\boldsymbol{\omega}_{x_{1} y_{1} z_{1}}
\end{gathered}
$$


The linear velocity of the hand is

$$
\dot{\boldsymbol{r}}_{w}=\left(\dot{\boldsymbol{r}}_{w}\right)_{r e l}+\boldsymbol{\omega}_{x_{1} y_{1} z_{1}} \times \boldsymbol{r}_{w}=\boldsymbol{\omega}_{x_{1} y_{1} z_{1}} \times \boldsymbol{r}_{w}
$$

where the relative velocity term vanishes because $x_{w}, y_{w}$, and $z_{w}$ are constant.

The kinetic energy of the hand is

$$
T_{m}=\frac{1}{2} m_{w} \dot{\boldsymbol{r}}_{w} \cdot \dot{\boldsymbol{r}}_{w}+\frac{1}{2} \boldsymbol{\omega}_{w}^{T}\left[\begin{array}{ccc}
I_{x w} & 0 & 0 \\
0 & I_{y w} & 0 \\
0 & 0 & I_{z w}
\end{array}\right] \boldsymbol{\omega}_{w}
$$

The potential energy of the hand, $V_{w}$, is the sum of the potential energy due to gravity and the stiffness of the wrist joint. The gravitational component is obtained by transforming the position of the body into the inertial $\boldsymbol{X}_{0} \boldsymbol{Y}_{0} \boldsymbol{Z}_{0}$ frame and taking the vertical $\left(Y_{0_{w}}\right)$ component:

$$
\begin{gathered}
\boldsymbol{r}_{w_{X_{0} Y_{0} Z_{0}}}=R_{1}^{T} \boldsymbol{r}_{w} \\
V_{w}=m_{w} g Y_{0_{w}}+\frac{1}{2} K_{w} \theta^{2}
\end{gathered}
$$

The Lagrangian of the hand is then simply

$$
L_{w}=T_{w}-V_{w}
$$

Body 2. Gyroscope Motor: The position of the COM (in the $\boldsymbol{x}_{1} \boldsymbol{y}_{1} \boldsymbol{z}_{1}$ frame) and the angular velocity (in the $\boldsymbol{x y z}$ frame) of the motor are

$$
\begin{gathered}
\boldsymbol{r}_{m}=\left[\begin{array}{ccc}
x_{p} & y_{p} & z_{p}
\end{array}\right]^{T}+R_{2}^{T} R_{3}^{T}\left[\begin{array}{lll}
0 & r_{m} & 0
\end{array}\right]^{T} \\
\boldsymbol{\omega}_{m}=\left[\begin{array}{lll}
0 & 0 & \dot{\phi}
\end{array}\right]^{T}+R_{3} R_{2} \boldsymbol{\omega}_{\boldsymbol{x}_{1} \boldsymbol{y}_{1} z_{1}}
\end{gathered}
$$

The linear velocity of the motor is 


$$
\dot{\boldsymbol{r}}_{m}=\left(\dot{r}_{m}\right)_{r e l}+\omega_{\boldsymbol{x}_{1} \boldsymbol{y}_{1} \mathbf{z}_{1}} \times \boldsymbol{r}_{m}
$$

The kinetic energy of the motor is

$$
T_{m}=\frac{1}{2} m_{m} \dot{\boldsymbol{r}}_{m} \cdot \dot{\boldsymbol{r}}_{m}+\frac{1}{2} \boldsymbol{\omega}_{m}^{T}\left[\begin{array}{ccc}
I_{x m} & 0 & 0 \\
0 & I_{y m} & 0 \\
0 & 0 & I_{z m}
\end{array}\right] \boldsymbol{\omega}_{m}
$$

The potential energy of the motor, $V_{m}$, is due to gravity, and it can be expressed by transforming the position of the body into the inertial $\boldsymbol{X}_{0} \boldsymbol{Y}_{0} \boldsymbol{Z}_{0}$ frame and taking the vertical $\left(Y_{0_{m}}\right)$ component:

$$
\begin{gathered}
\boldsymbol{r}_{m_{\boldsymbol{X}_{0} \boldsymbol{Y}_{0} \boldsymbol{Z}_{0}}}=R_{1}^{T} \boldsymbol{r}_{m} \\
V_{m}=m_{m} g Y_{0_{m}}
\end{gathered}
$$

The Lagrangian of the motor is then simply

$$
L_{m}=T_{m}-V_{m}
$$

Body 3. Gyroscope Flywheel: The position of the COM (in the $\boldsymbol{x}_{1} \boldsymbol{y}_{1} \boldsymbol{z}_{1}$ frame) and the angular velocity (in the $\boldsymbol{x y z}$ frame) of the flywheel are

$$
\begin{gathered}
\boldsymbol{r}_{f}=\left[\begin{array}{lll}
x_{p} & y_{p} & z_{p}
\end{array}\right]^{T}+R_{2}^{T} R_{3}^{T}\left[\begin{array}{lll}
0 & r_{f} & 0
\end{array}\right]^{T} \\
\boldsymbol{\omega}_{f}=\left[\begin{array}{lll}
0 & \Omega & \dot{\phi}
\end{array}\right]^{T}+R_{3} R_{2} \boldsymbol{\omega}_{x_{1} y_{1} z_{1}}
\end{gathered}
$$

The linear velocity of the flywheel is

$$
\dot{\boldsymbol{r}}_{f}=\left(\dot{\boldsymbol{r}}_{f}\right)_{r e l}+\omega_{x_{1} y_{1} z_{1}} \times \boldsymbol{r}_{f}
$$

The kinetic energy of the flywheel is 


$$
T_{f}=\frac{1}{2} m_{f} \dot{\boldsymbol{r}}_{f} \cdot \dot{\boldsymbol{r}}_{f}+\frac{1}{2} \boldsymbol{\omega}_{f}^{T}\left[\begin{array}{ccc}
I_{x f} & 0 & 0 \\
0 & I_{y f} & 0 \\
0 & 0 & I_{z f}
\end{array}\right] \boldsymbol{\omega}_{f}
$$

The potential energy of the flywheel, $V_{f}$, is the sum of the potential energy due to gravity and the stiffness about the precession axis, and it can be expressed by transforming the position of the body into the inertial $\boldsymbol{X}_{0} \boldsymbol{Y}_{0} \boldsymbol{Z}_{0}$ frame and taking the vertical $\left(Y_{0_{f}}\right)$ component:

$$
\begin{gathered}
\boldsymbol{r}_{f_{\boldsymbol{X}_{0} \boldsymbol{Y}_{0} \boldsymbol{Z}_{0}}}=R_{1}^{T} \boldsymbol{r}_{f} \\
V_{f}=m_{f} g Y_{0_{f}}+(1 / 2) K_{p} \phi^{2}
\end{gathered}
$$

The Lagrangian of the flywheel is then simply

$$
L_{f}=T_{f}-V_{f}
$$

The Lagrangian of the entire system is

$$
L=L_{w}+L_{m}+L_{f}
$$

\section{B.1.3 Equations of Motion}

The equations of motion for the two DOF (wrist angle $\theta$ and precession angle $\varnothing$ ) were calculated using Lagrange's equations:

$$
\begin{aligned}
& \frac{\partial}{\partial t}\left(\frac{\partial L}{\partial \dot{\theta}}\right)-\left(\frac{\partial L}{\partial \theta}\right)=T_{w}-D_{w} \dot{\theta} \\
& \frac{\partial}{\partial t}\left(\frac{\partial L}{\partial \dot{\varnothing}}\right)-\left(\frac{\partial L}{\partial \varnothing}\right)=T_{p}-D_{p} \dot{\varnothing}
\end{aligned}
$$

Lagrange's equation was implemented in Matlab using the Euler-Lagrange tool package [69] as follows: 


$$
\begin{aligned}
& L=L_{g}+L_{m}+L_{w} \\
& x=\left\{\begin{array}{llll}
\theta & \dot{\theta} & \phi & \dot{\phi}
\end{array}\right\} \\
& p=\left\{I_{x f} I_{y f} I_{z f} I_{x w} I_{y w} I_{z w} I_{x m} I_{y m} I_{z m} g \Omega m_{f} m_{w} x_{p} y_{p} z_{p} x_{w} y_{w} z_{w} r_{f} T_{w} D_{w} K_{w} T_{p} D_{p} K_{p} m_{m} r_{m}\right\} ; \\
& R=0 \text {; } \\
& Q_{i}=\left\{\begin{array}{ll}
-D_{w} \dot{\theta} & -D_{p} \dot{\phi}
\end{array}\right\} \\
& Q_{e}=\left\{\begin{array}{ll}
T_{w} & T_{p}
\end{array}\right\} \\
& V F=\text { EulerLagrange }\left(L, x, Q_{i}, Q_{e}, R, p\right) \\
& V F_{1}=\operatorname{subs}\left(V F, \quad\left[\begin{array}{llll}
\theta & \phi & \dot{\theta} & \dot{\phi}
\end{array}\right], \quad\left[\begin{array}{llll}
x_{1} & x_{2} & x_{3} & x_{4}
\end{array}\right]\right) ; \\
& \dot{x}=\left[V F_{1}(1) ; \quad V F_{1}(3) ; \quad V F_{1}(2) ; \quad V F_{1}(4)\right]=\left[\begin{array}{lll}
\dot{x}_{1} ; & \dot{x}_{2} ; \quad \dot{x}_{3} ; \quad \dot{x}_{4}
\end{array}\right]
\end{aligned}
$$

where $x$ is the vector of generalized coordinates, $p$ is the system parameters, $R$ is the friction term, $Q_{i}$ is the internal generalized forces, and $Q_{e}$ is the external generalized forces. These functions find the non-linear equations of motion for the model as first-order state equations, where $x_{1}=\theta, x_{2}=\phi, x_{3}=\dot{\theta}$, and $x_{4}=\dot{\phi}$ are the states.

\section{B.2 Linearization}

The process described above produces nonlinear EOM that can be summarized as $\dot{\boldsymbol{x}}=$ $\boldsymbol{f}(\boldsymbol{x}, \boldsymbol{u})$, where $\boldsymbol{x}$ is the vector of states and $\boldsymbol{u}$ is the vector of inputs. The model has four states $\left(\boldsymbol{x}=\left[\begin{array}{llll}\theta & \dot{\theta} & \phi & \dot{\phi}\end{array}\right]^{T}\right)$ and two inputs:

$$
\boldsymbol{u}=\boldsymbol{u}^{e q}+\delta \boldsymbol{u}=\left[\begin{array}{c}
T_{w}^{e q} \\
T_{p}^{e q}
\end{array}\right]+\left[\begin{array}{c}
T_{w}^{p e r t} \\
0
\end{array}\right]=\left[\begin{array}{c}
T_{w} \\
T_{p}
\end{array}\right]
$$


where $T_{w}^{e q}$ and $T_{p}^{e q}$ are the equilibrium torques (Table B-3) about the wrist joint and precession axes, respectively, and $T_{w}^{p e r t}$ is the tremorogenic (sinusoidal) input into the linear system, applied about the wrist joint axis. In general, the final output of the simulation, $y$, depends on the states and inputs: $\boldsymbol{y}=g(\boldsymbol{x}, \boldsymbol{u})$. In our case, the output of interest is one of the states $\left(x_{1}=\theta\right)$, so $\boldsymbol{g}(\boldsymbol{x}, \boldsymbol{u})=x_{1}$

Table B-3: Equilibrium Torques for each Gyroscope Orientation

\begin{tabular}{|c|c|c|c|c|}
\cline { 2 - 5 } \multicolumn{1}{c|}{} & \multicolumn{3}{c|}{$T_{w}^{e q}$} & $T_{p}^{e q}$ \\
\cline { 2 - 5 } \multicolumn{1}{c|}{} & FE & RUD & PS & FE, RUD, and PS \\
\hline O1 & $g\left(m_{f} x_{p}+m_{m} x_{p}+m_{w} x_{w}\right)$ & 0 & $-g\left(m_{f} z_{p}+m_{m} z_{p}+m_{w} z_{w}\right)$ & 0 \\
\hline O2 & $g\left(m_{f} x_{p}+m_{m} x_{p}+m_{w} x_{w}\right)$ & 0 & $-g\left(m_{f} z_{p}+m_{m} z_{p}+m_{w} z_{w}\right)$ & 0 \\
\hline O3 & $g\left(m_{f} x_{p}+m_{m} x_{p}+m_{w} x_{w}+m_{f} r_{f}+m_{m} r_{m}\right)$ & 0 & $-g\left(m_{f} z_{p}+m_{m} z_{p}+m_{w} z_{w}\right)$ & $g\left(m_{f} r_{f}+m_{m} r_{m}\right)$ \\
\hline O4 & $g\left(m_{f} x_{p}+m_{m} x_{p}+m_{w} x_{w}+m_{f} r_{f}+m_{m} r_{m}\right)$ & 0 & $-g\left(m_{f} z_{p}+m_{m} z_{p}+m_{w} z_{w}\right)$ & 0 \\
\hline O5 & $g\left(m_{f} x_{p}+m_{m} x_{p}+m_{w} x_{w}\right)$ & 0 & $-g\left(m_{f} r_{f}+m_{m} r_{m}+m_{f} z_{p}+m_{m} z_{p}+m_{w} z_{w}\right)$ & 0 \\
\hline O6 & $g\left(m_{f} x_{p}+m_{m} x_{p}+m_{w} x_{w}\right)$ & 0 & $-g\left(m_{f} r_{f}+m_{m} r_{m}+m_{f} z_{p}+m_{m} z_{p}+m_{w} z_{w}\right)$ & $-g\left(m_{f} r_{f}+m_{m} r_{m}\right)$ \\
\hline
\end{tabular}

The following LTI SS system defines the local linearization of $\dot{\boldsymbol{x}}=\boldsymbol{f}(\boldsymbol{x}, \boldsymbol{u})$ and $\boldsymbol{y}=$ $\boldsymbol{g}(\boldsymbol{x}, \boldsymbol{u})$ about the equilibrium point $\left(\boldsymbol{x}^{e q}, \boldsymbol{u}^{e q}\right)$ :

$$
\begin{aligned}
& \delta \dot{\boldsymbol{x}}=A \delta \boldsymbol{x}+B \delta \boldsymbol{u} \\
& \delta \boldsymbol{y}=C \delta \boldsymbol{x}+D \delta \boldsymbol{u}
\end{aligned}
$$

where $A=\frac{\partial f}{\partial \boldsymbol{x}}\left(\boldsymbol{x}^{e q}, \boldsymbol{u}^{e q}\right), B=\frac{\partial \boldsymbol{f}}{\partial \boldsymbol{u}}\left(\boldsymbol{x}^{e q}, \boldsymbol{u}^{e q}\right), C=\frac{\partial \boldsymbol{g}}{\partial \boldsymbol{x}}\left(\boldsymbol{x}^{e q}, \boldsymbol{u}^{e q}\right), D=\frac{\partial \boldsymbol{g}}{\partial \boldsymbol{u}}\left(\boldsymbol{x}^{e q}, \boldsymbol{u}^{e q}\right)$.

The desired equilibrium point was chosen to be the system at rest, with zero wrist angle $(\theta=0)$ and zero precession angle $(\phi=0)$, i.e. $x^{e q}=\left[\begin{array}{llll}0 & 0 & 0 & 0\end{array}\right]$. Note that this equilibrium point is valid for all 18 cardinal configurations (Figure 2-3). The input required to achieve this equilibrium point was calculated for each configuration as follows. To be an equilibrium point, $\dot{\boldsymbol{x}}$ must be zero, so $\boldsymbol{x}^{e q}$ and $\boldsymbol{u}^{e q}$ must satisfy $\boldsymbol{f}\left(\boldsymbol{x}^{e q}, \boldsymbol{u}^{e q}\right)=0$. Since $\boldsymbol{x}^{e q}$ is known, we solved this equation for $\boldsymbol{u}^{e q}$, yielding the values of $T_{w}^{e q}$ and $T_{p}^{e q}$ required to achieve equilibrium (Table B-3). 
Therefore, when $\boldsymbol{x}(t)=\boldsymbol{x}^{e q}$ and $\boldsymbol{u}(t)=\boldsymbol{u}^{e q}$, the output is $\boldsymbol{y}=\boldsymbol{y}^{e q}=\boldsymbol{g}\left(\boldsymbol{x}^{e q}, \boldsymbol{u}^{e q}\right)=\mathbf{0}$ for all time. If instead the input is $\boldsymbol{u}(t)=\boldsymbol{u}^{e q}+\delta \boldsymbol{u}(t)$ and/or the initial condition is $\boldsymbol{x}(0)=\boldsymbol{x}^{e q}+$ $\delta \boldsymbol{x}^{e q}$, the output will be close to (but not equal to) $\boldsymbol{y}^{e q}$.

The linearization was implemented in Matlab as follows:

$$
\begin{gathered}
x=\left[x_{1} ; x_{2} ; x_{3} ; x_{4}\right] ; \\
u=\left[T_{w} ; T_{p}\right] ; \\
f=\dot{x} ; \\
g=x_{1} ; \\
A_{t}=j \operatorname{jacobian}(f, x) ; \\
B_{t}=j \operatorname{jacobian}(f, u) ; \\
C_{t}=j \operatorname{jacobian}(g, x) ; \\
D_{t}=\operatorname{jacobian}(g, u) ;
\end{gathered}
$$

where $A_{t}, B_{t}, C_{t}$, and $D_{t}$ are intermediate matrices used to calculate the SS matrices; when evaluated at the equilibrium points (states and torques) required for the given gyroscope orientation, $A_{t}, B_{t}, C_{t}$, and $D_{t}$ become the linearized SS matrices $A, B, C$, and $D$. 


\section{APPENDIX C. CODE TO GENERATE STATE SPACE MATRICES}

\section{C.1 Description}

A Matlab function named Complete_1DOF.m uses the method described in Appendix B to calculate the SS EOMs of the model. The first step when using Complete_1DOF.m is to set the desired wrist DOF and gyroscope orientation. The wrist DOF is set by making Rotation equal to 'FE', 'RUD', or 'PS' for a desired wrist DOF of flexion-extension, radial-ulnar deviation, or pronation-supination respectively. The gyroscope orientation can be set as one of the six cardinal orientations (Figure 2-2) by making orientation equal to 1, 2, 3, 4, 5, or 6 for a desired orientation of O1, O2, O3, O4, O5, or O6 respectively. Complete_1DOF.m was used to obtain the EOMs of all 18 of the cardinal configurations (Figure 2-3). This code must be run before Sensitivity.m or LINvsNL_Main.m are run because they use the SS matrices calculated by Complete_1DOF.m. Before running Sensitivity.m make sure $\mathrm{G}=\mathrm{x} 1$ in the linearization section of Complete_1DOF.m. Additionally, to run LINvsNL_Main.m make sure that $\mathrm{G}=[\mathrm{x} 1 ; \mathrm{x} 2]$.

\section{C.2 Complete_1DOF.m}

clear

clc

syms th theta(t) thdot phi phii(t) phidot

syms Ix Iy Iz mg rg \%gyro flywheel

syms Imx Imy Imz mm rm \%gyro motor 
syms xg yg zg lam kp Dp Tp \%general gyro

syms g

syms Iwx Iwy Iwz mw xw yw zw Tw kw Dw \% Hand properties

syms b1 b2 b3

$\% \% \% \% \% \% \% \% \% \% \% \%$ SET DIRECTION OF HAND ROTATION \%\%\%\%\%\%\%\%\%\%\%\%

Rotation $=$ 'FE' $\%$ This sets 1 DOF the hand rotates about

if strcmp(Rotation, 'FE')

$\mathrm{R} 1=[\cos (\mathrm{th}) \sin (\mathrm{th}) 0 ;-\sin (\mathrm{th}) \cos (\mathrm{th}) \mathbf{0}$; 00 1]; \% R_X0Y0Z0_to_x1y1z1

w_xlylz1 $=[0 ; 0 ;$ thdot $] ; \%$ Ang vel of frame x1y1z1

elseif strcmp(Rotation, 'RUD')

$\mathrm{R} 1=\left[\cos (\right.$ th) $0-\sin ($ th $) ; 010 ; \sin ($ th $) 0 \cos ($ th $)] ; \%$ R_X $0 Y 0 Z 0 \_t o \_x 1 y 1 z 1$

W_x1y1z1 $=[0 ;$ thdot;0]; \% Ang vel of frame x1y1z1

elseif strcmp(Rotation, 'PS')

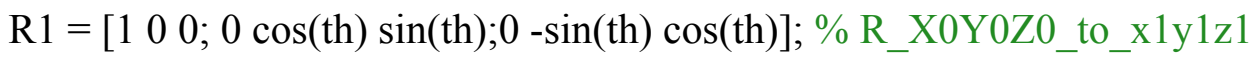

w_x1y1z1 $=[$ thdot;0;0]; \% Ang vel of frame x1y1z1

else rotation_error $=1$

end

$\% \% \% \% \% \% \% \% \% \% \%$ Rotation matrices to go from hand-fixed to body-fixed $\% \% \% \% \% \% \% \% \%$

$\mathrm{R} 21=[\cos (\mathrm{b} 1) 0-\sin (\mathrm{b} 1) ; 010 ; \sin (\mathrm{b} 1) 0 \cos (\mathrm{b} 1)]$

$\mathrm{R} 22=[\cos (\mathrm{b} 2) \sin (\mathrm{b} 2) 0 ;-\sin (\mathrm{b} 2) \cos (\mathrm{b} 2) 0 ; 0001]$

$\mathrm{R} 23=[\cos (\mathrm{b} 3) 0-\sin (\mathrm{b} 3) ; 010 ; \sin (\mathrm{b} 3) 0 \cos (\mathrm{b} 3)]$

$\mathrm{R} 2=\mathrm{R} 23 * \mathrm{R} 22 * \mathrm{R} 21 ; \% \mathrm{R}$-x1y1z1_to_XYZ

$\mathrm{R} 3=[\cos (\mathrm{phi}) \sin (\mathrm{phi}) 0 ;-\sin (\mathrm{phi}) \cos (\mathrm{phi}) 0 ; 001] ; \%$ R_XYZ_to_xyz 
$\% \% \% \% \% \% \% \% \% \%$ Rotation matrices to go from body-fixed to inertial $\% \% \% \% \% \% \% \% \% \% \%$

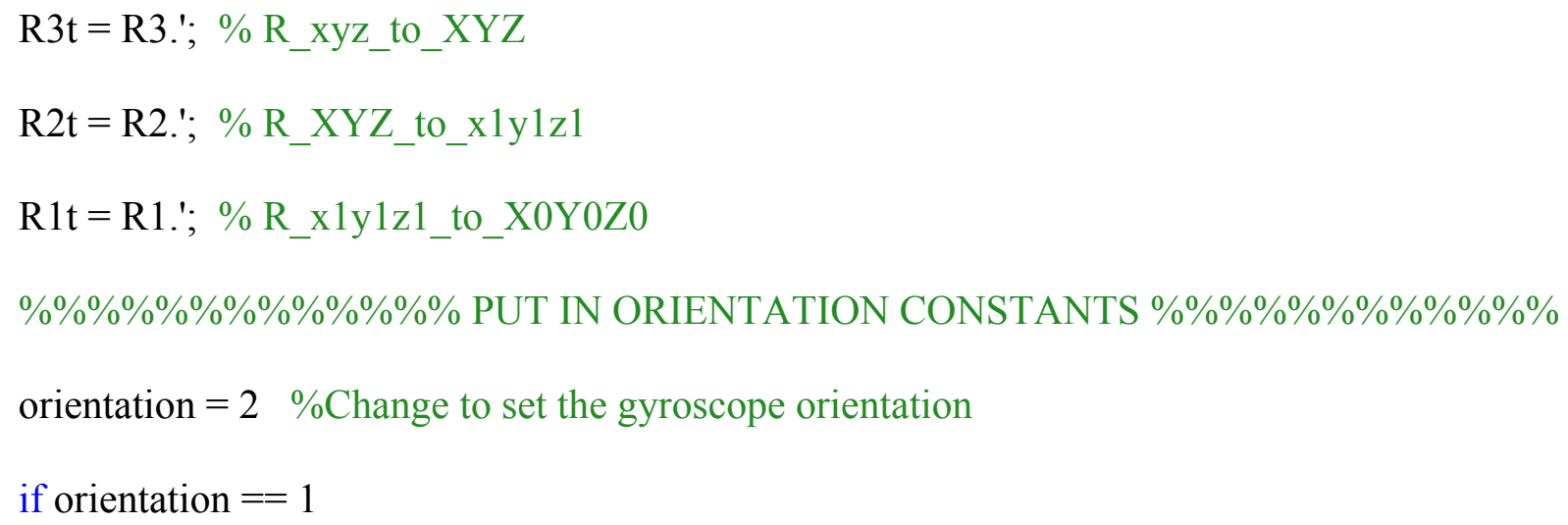




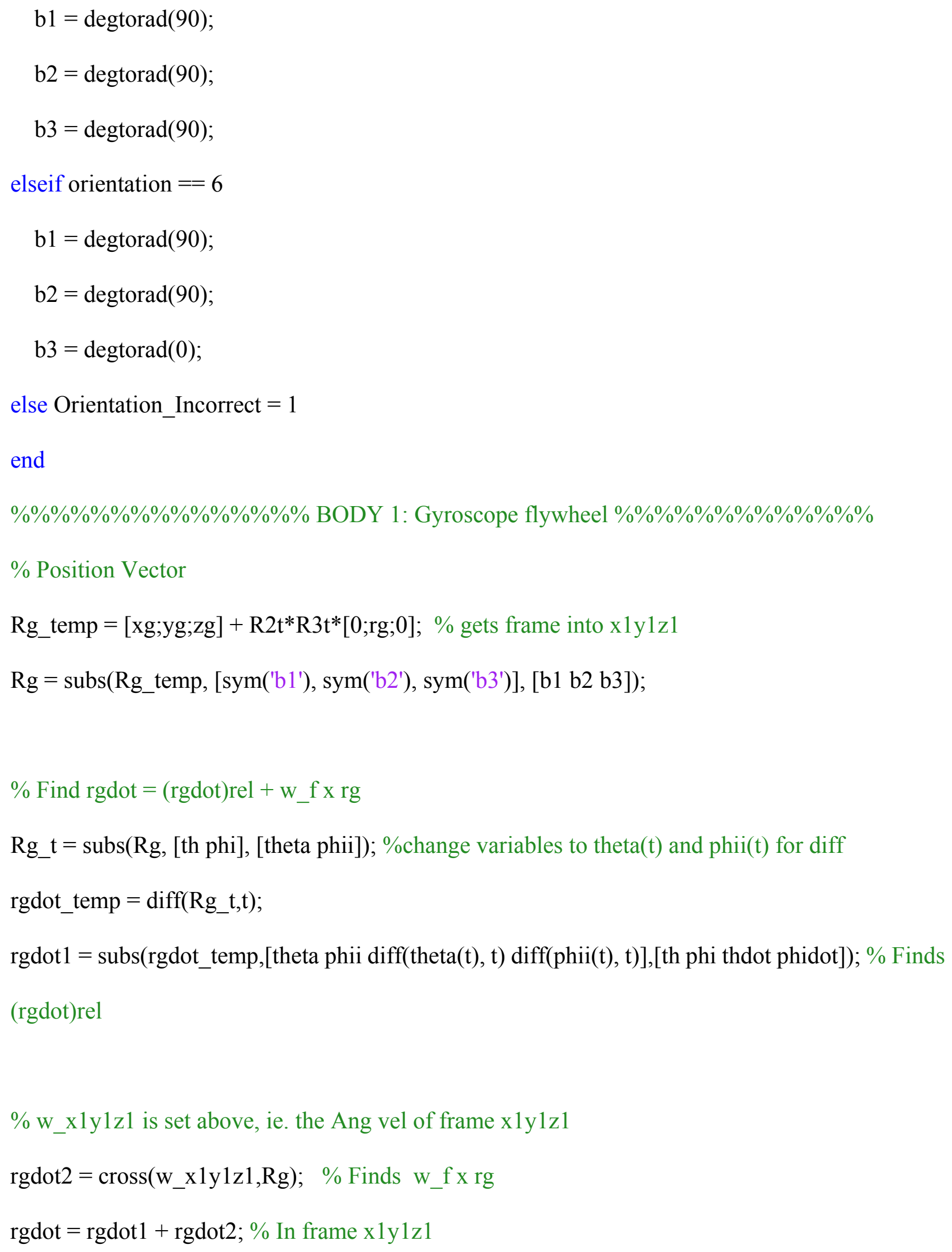


$\%$ Find angular velocity of Gyro Flywheel (body-fixed)

$\mathrm{wg}=[0 ;$ lam;phidot $]+\mathrm{R} 3 * \mathrm{R} 2 * \mathrm{w}_{-} \mathrm{x} 1 \mathrm{y} 1 \mathrm{z} 1$

$\mathrm{wg}=\operatorname{subs}(\mathrm{wg},[\operatorname{sym}(' \mathrm{~b} 1$ '), sym('b2'), sym('b3')], [b1 b2 b3]);

$\%$ Find $\mathrm{KE}=(1 / 2) * \mathrm{mg} * \operatorname{rgdot} * \operatorname{rgdot}+(1 / 2) * \mathrm{wg}^{\prime *} \mathrm{I}^{*} \mathrm{wg}$

$\mathrm{I}=[\mathrm{Ix} 00$ 0; 0 Iy $0 ; 00 \mathrm{Iz}] ; \%$ Inertia of flywheel

$\mathrm{Tg}=\operatorname{simplify}(\operatorname{expand}((1 / 2) * \mathrm{mg} *(\operatorname{rgdot}(1) * \operatorname{rgdot}(1)+\operatorname{rgdot}(2) * \operatorname{rgdot}(2)+\operatorname{rgdot}(3) * \operatorname{rgdot}(3))+$ $\left.\left.(1 / 2)^{*} w g .{ }^{*} I^{*} w g\right)\right)$;

$\%$ Find $\mathrm{PE}=\mathrm{mgh}+(1 / 2) \mathrm{k}^{*} \mathrm{phi}^{\wedge} 2$

Rg_X0Y0Z0 = R1t*Rg;

$\mathrm{Y} 0=\mathrm{Rg} \_\mathrm{X} 0 \mathrm{Y} 0 \mathrm{Z} 0(2)$;

$\mathrm{Vg}=\operatorname{simplify}\left(\operatorname{expand}\left(\mathrm{mg}{ }^{*} \mathrm{~g}^{*} \mathrm{Y} 0+(1 / 2) * \mathrm{kp}^{*} \mathrm{phi}^{\wedge} 2\right)\right)$

$\%$ Find lagrangian for gyroscope

$\operatorname{Lg}=\mathrm{Tg}-\mathrm{Vg}$

\%\%\%\%\%\%\%\%\%\%\%\%\%\% BODY 2: Gyroscope Motor \%\%\%\%\%\%\%\%\%\%\%\%\%\%

$\%$ Position Vector

$\mathrm{Rm}=[\mathrm{xg} ; \mathrm{yg} ; \mathrm{zg}]+\mathrm{R} 2 \mathrm{t}^{*} \mathrm{R} 3 \mathrm{t}^{*}[0 ; \mathrm{rm} ; 0] ; \%$ gets frame into x1y1z1

$\mathrm{Rm}=\operatorname{subs}(\mathrm{Rm},[\operatorname{sym}(' \mathrm{~b} 1$ '), sym('b2'), sym('b3')], [b1 b2 b3]);

$\%$ Find rgdot $=($ rgdot $)$ rel + w_f $x$ rg

$R m_{-} t=\operatorname{subs}(R m$, [th phi], [theta phii]); \%change variables to theta $(t)$ and phii $(t)$ for diff 
rmdot_temp $=\operatorname{diff}\left(\mathrm{Rm} \_\mathrm{t}, \mathrm{t}\right)$;

rmdot $1=\operatorname{subs}($ rmdot_temp, [theta phii $\operatorname{diff}(\operatorname{theta}(\mathrm{t}), \mathrm{t}) \operatorname{diff}(\operatorname{phii}(\mathrm{t}), \mathrm{t})],[$ th phi thdot phidot] $) ; \%$ Finds (rgdot)rel

$\%$ w_xly1z1 is set above, ie. the Ang vel of frame x1y1z1

$\operatorname{rmdot} 2=\operatorname{cross}\left(\mathrm{w} \_\mathrm{x} 1 \mathrm{y} 1 \mathrm{z} 1, \mathrm{Rm}\right) ; \%$ Finds $\mathrm{w} \_\mathrm{f} x \mathrm{rg}$

$\operatorname{rmdot}=\operatorname{rmdot} 1+\operatorname{rmdot} 2 ; \%$ In frame $\mathrm{x} 1 \mathrm{y} 1 \mathrm{z} 1$

\% Find angular velocity of Gyro Flywheel (body-fixed)

$\mathrm{wm}=[0 ; 0 ;$ phidot $]+\mathrm{R} 3 * \mathrm{R} 2 * \mathrm{w} \_\mathrm{x} 1 \mathrm{y} 1 \mathrm{z} 1$

wm $=\operatorname{subs}(w m,[\operatorname{sym}(' b 1 '), \operatorname{sym}(' b 2 '), \operatorname{sym}(' b 3 ')],[b 1$ b2 b3] $)$;

$\%$ Find $\mathrm{KE}=(1 / 2) * \mathrm{~mm} * \mathrm{rmdot}^{*} \mathrm{rmdot}+(1 / 2) * \mathrm{wm}^{\prime}{ }^{*} \mathrm{Im}^{*} \mathrm{wm}$

$\operatorname{Im}=[\operatorname{Imx} 0$ 0; 0 Imy 0; $00 \mathrm{Imz}] ; \%$ Inertia of flywheel

$\mathrm{Tm}=\operatorname{simplify}(\operatorname{expand}((1 / 2) * \mathrm{~mm} *(\operatorname{rmdot}(1) * \operatorname{rmdot}(1)+\operatorname{rmdot}(2) * \operatorname{rmdot}(2)+\operatorname{rmdot}(3) * \operatorname{rmdot}(3))$

$\left.\left.+(1 / 2)^{*} \mathrm{wm} .{ }^{\prime *} \operatorname{Im}^{*} \mathrm{wm}\right)\right)$;

$\%$ Find $\mathrm{PE}=\mathrm{mgh}$

$\mathrm{Rm} \_\mathrm{X} 0 \mathrm{Y} 0 Z 0=\mathrm{R} 1 \mathrm{t}^{*} \mathrm{Rm}$;

$\mathrm{Y} 0 \mathrm{~m}=\mathrm{Rm} \_\mathrm{X} 0 \mathrm{Y} 0 \mathrm{Z} 0(2)$;

$\mathrm{Vm}=\operatorname{simplify}\left(\operatorname{expand}\left(\mathrm{mm}^{*} \mathrm{~g} * \mathrm{Y} 0 \mathrm{~m}\right)\right)$

$\%$ Find lagrangian for gyroscope

$\mathrm{Lm}=\mathrm{Tm}-\mathrm{Vm} ;$ 
\%\%\%\%\%\%\%\%\%\%\%\%\%\%\%\%\%\%\% BODY 3: HAND \%\%\%\%\%\%\%\%\%\%\%\%\%\%\%\%\%

$\%$ Position Vector

$\mathrm{Rw}=[\mathrm{xw} ; \mathrm{yw} ; \mathrm{zw}] ; \%$ frame in $\mathrm{x} 1 \mathrm{y} 1 \mathrm{z} 1$

$\%$ Find rwdot $=($ rwdot $) r e l+w_{-}$f $x$ rw

$\mathrm{Rw}_{\mathrm{C}} \mathrm{t}=\operatorname{subs}(\mathrm{Rw}$, [th phi], [theta phii]); \%change variables to theta $(\mathrm{t})$ and phii $(\mathrm{t})$ for diff

rwdot_temp $=\operatorname{diff}\left(\mathrm{Rw}_{-} \mathrm{t}, \mathrm{t}\right)$;

rwdot $1=\operatorname{subs}($ rwdot_temp, [theta phii $\operatorname{diff}($ theta(t), $t) \operatorname{diff}(p h i i(t), t)],[$ th phi thdot phidot] $) ; \%$ Finds (rgdot)rel

$\%$ w_x $1 y 1 z 1$ is set above, ie. the Ang vel of frame xly1z1

$\operatorname{rwdot} 2=\operatorname{cross}\left(\mathrm{w} \_\mathrm{x} 1 \mathrm{y} 1 \mathrm{z} 1, \mathrm{Rw}\right) ; \%$ Finds $\mathrm{w}_{-} \mathrm{f} \mathrm{x}$ rg

$\operatorname{rwdot}=\operatorname{rwdot} 1+\operatorname{rwdot} 2 ; \%$ In frame x1y1z1

$\%$ Find angular velocity of Hand (body-fixed)

$\mathrm{ww}=\mathrm{w} \_\mathrm{x} 1 \mathrm{y} 1 \mathrm{z} 1$

$\%$ Find $\mathrm{KE}=(1 / 2) * \mathrm{mw} * \mathrm{rwdot} * \mathrm{rwdot}+(1 / 2) * \mathrm{ww}^{\prime *} \mathrm{Iw}^{*} \mathrm{ww}$

$\mathrm{Iw}=[\operatorname{Iwx} 00 ; 0$ Iwy $0 ; 00 \mathrm{Iwz}] ; \%$ Inertia of flywheel

Twrist $=\operatorname{simplify}(\operatorname{expand}((1 / 2) * m w *(\operatorname{rwdot}(1) * \operatorname{rwdot}(1)+\operatorname{rwdot}(2) * \operatorname{rwdot}(2)+$ $\left.\left.\operatorname{rwdot}(3) * \operatorname{rwdot}(3))+(1 / 2)^{*} w w . * \mathrm{Iw}^{*} \mathrm{ww}\right)\right)$;

$\%$ Find $\mathrm{PE}=\mathrm{mgh}+(1 / 2) \mathrm{k}^{*} \mathrm{th}^{\wedge} 2$

Rw_X0Y0Z0 = R1t*Rw;

$\mathrm{Y} 0=\mathrm{Rw}_{-} \mathrm{X} 0 \mathrm{Y} 0 \mathrm{Z} 0(2)$ 
$\mathrm{Vw}=\operatorname{simplify}\left(\operatorname{expand}\left(\mathrm{mw}^{*} \mathrm{~g} * \mathrm{Y} 0+(1 / 2) * \mathrm{kw}^{*} \mathrm{th}^{\wedge} 2\right)\right)$

$\%$ Find lagrangian for wrist

$\mathrm{Lw}=$ Twrist $-\mathrm{Vw}$;

$\% \% \% \% \% \% \% \% \% \% \% \% \% \% \% \% \%$ Equations of Motion $\% \% \% \% \% \% \% \% \% \% \% \% \% \% \% \% \% \% \%$

Lagrange $=\mathrm{Lg}+\mathrm{Lm}+\mathrm{Lw}$;

$\mathrm{X}=\{$ th thdot phi phidot $\}$

par $=\{$ Ix Iy Iz Iwx Iwy Iwz g lam mg mw xg yg zg xw yw zw rg Tw Dw kw Tp kp Dp b1 b2 b3 $\operatorname{Imx} \operatorname{Imy} \operatorname{Imz} \mathrm{mm} \operatorname{rm}\}$;

$\mathrm{R}=0$

Q_i $=\left\{-D w^{*}\right.$ thdot, - Dp*phidot $\}$;

$\mathrm{Q} \_\mathrm{e}=\{\mathrm{Tw} \mathrm{Tp}\}$

VF = EulerLagrange(Lagrange,X,Q_i,Q_e,R,par);

$\%$ Rearrange so $\mathrm{x} 1=$ th, $\mathrm{x} 2=$ phi, $\mathrm{x} 3=$ thdot, $\mathrm{x} 4=$ phidot

syms x $1 \times 2 \times 3 \times 4$

temp1 $=\operatorname{subs}(\mathrm{VF},[$ th phi thdot phidot $],[\mathrm{x} 1 \times 2 \times 3 \times 4])$;

$\mathrm{xdot}=[$ temp1(1);temp1(3);temp1(2);temp1(4)];

$\% \% \% \% \% \% \% \% \% \% \% \% \% \% \% \% \% \% \%$ \%LINEARIZATION $\% \% \% \% \% \% \% \% \% \% \% \% \% \% \% \% \% \% \%$

$\mathrm{x}=[\mathrm{x} 1 ; \mathrm{x} 2 ; \mathrm{x} 3 ; \mathrm{x} 4]$

$\mathrm{u}=[\mathrm{Tw} ; \mathrm{Tp}]$

$\mathrm{f}=\mathrm{xdot}$;

$\mathrm{G}=[\mathrm{x} 1 ; \mathrm{x} 2] ; \%$ This is needed to run LINvsNL_Main

$\% \mathrm{G}=\mathrm{x} 1$; 

A_temp $=$ jacobian $(f, x)$;
B_temp $=$ jacobian $(\mathrm{f}, \mathrm{u})$;
C_temp $=\operatorname{jacobian}(\mathrm{G}, \mathrm{x})$;
D_temp $=$ jacobian $(\mathrm{G}, \mathrm{u})$;

$\%$ Once equil. points are subbed in will have SS matrices

\%\%\%\%\%\%\%\%\%\%\%\%\%\%\%\% EQUILIBRIUM POINTS \%\%\%\%\%\%\%\%\%\%\%\%\%\%\%\%

$\% \% \% \% \% \% \% \% \% \% \%$ x equilibrium $\% \% \% \% \% \% \% \% \% \% \%$

x1_eq $=0$;

x2_eq $=0$;

x3_eq $=0$;

x4_eq=0;

$\% \% \% \% \% \% \% \% \% \% \%$ u equilibrium $\% \% \% \% \% \% \% \% \% \% \%$

if orientation $==1$

if strcmp(Rotation, 'FE')

Tw_eq $=g^{*}\left(m g^{*} x g+m m^{*} x g+m w^{*} x w\right)$;

Tp_eq $=0$

elseif strcmp(Rotation, 'RUD')

Tw_eq $=0$;

Tp_eq $=0$

elseif strcmp(Rotation, 'PS')

Tw_eq $=-g^{*}\left(\mathrm{mg}^{*} \mathrm{zg}+\mathrm{mm}^{*} \mathrm{zg}+\mathrm{mw}^{*} \mathrm{zw}\right)$;

Tp_eq $=0$;

end 


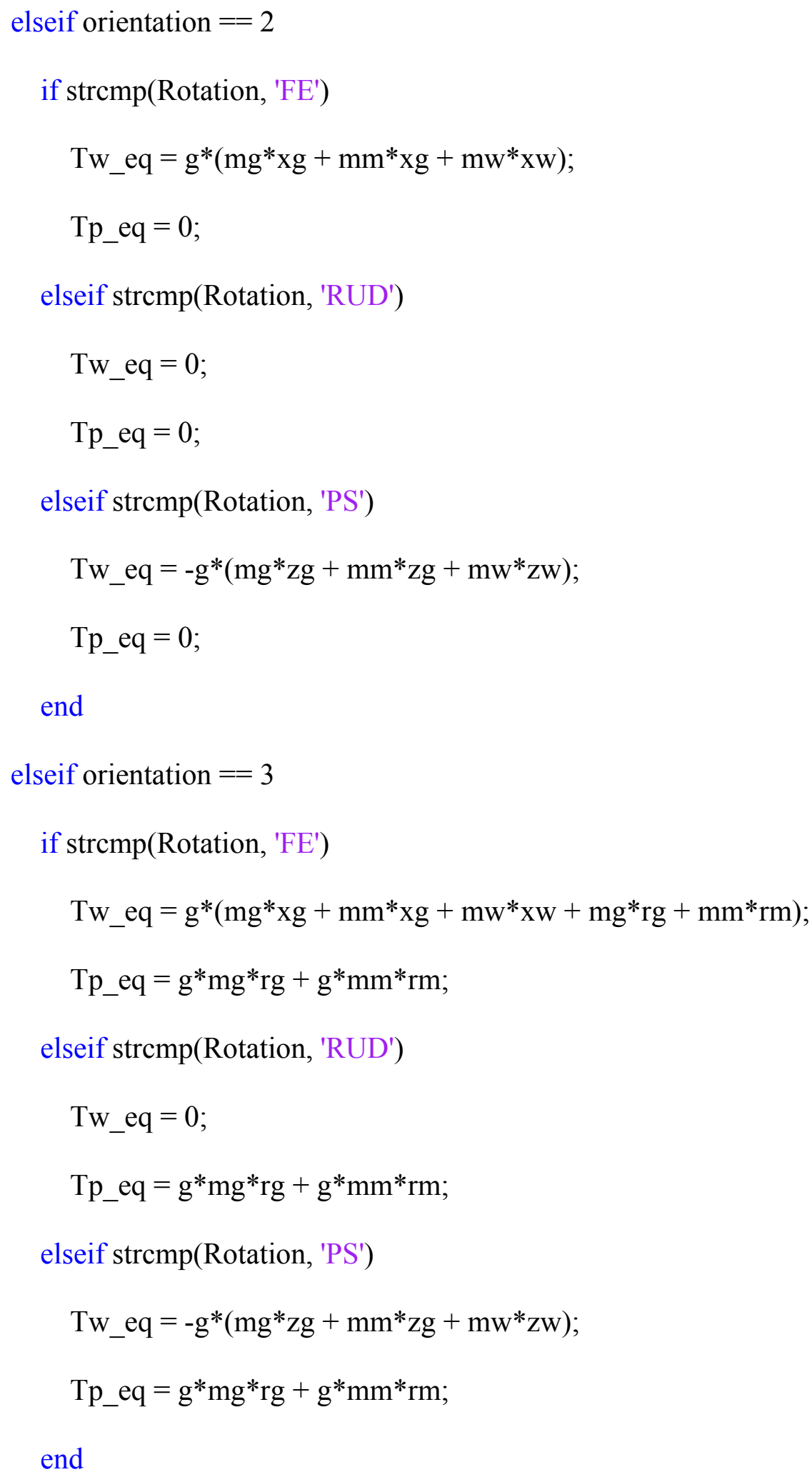




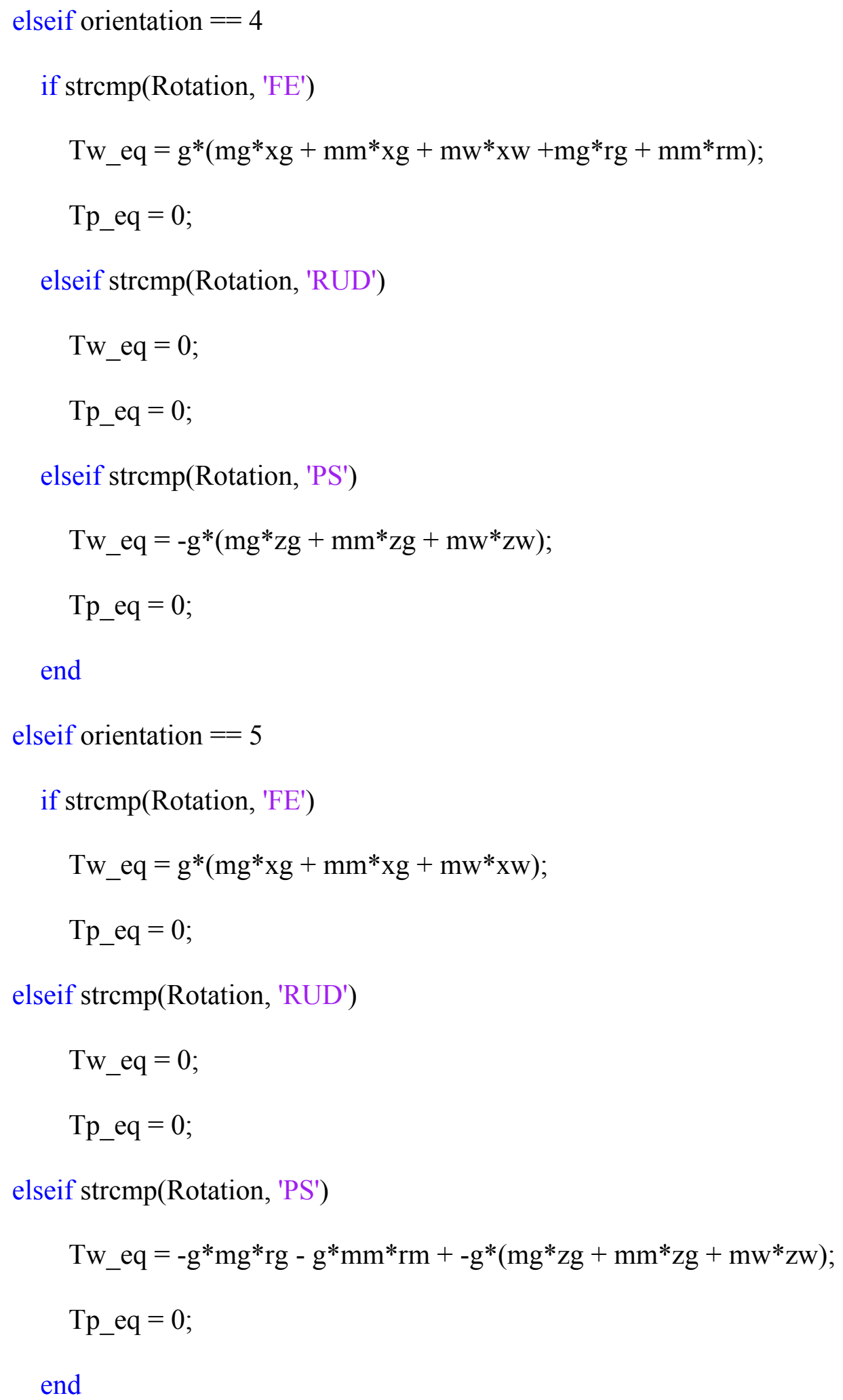


elseif orientation $==6$

if strcmp(Rotation, 'FE')

Tw_eq $=g^{*}\left(m g^{*} x g+m m^{*} x g+m w^{*} x w\right)$;

Tp_eq $=-g^{*} m g^{*}$ rg $-g * m m * r m$;

elseif strcmp(Rotation, 'RUD')

Tw_eq $=0$;

Tp_eq $=-g * m g * r g-g * m m * r m ;$

elseif strcmp(Rotation, 'PS')

Tw_eq $=-g * m g^{*}$ rg $-g * m m * r m+-g *\left(m g^{*} z g+m m^{*} z g+m w^{*} z w\right)$;

Tp_eq $=-g * m g *$ rg $-g * m m * r m$;

end

else Torque_Incorrect $=1$

end

$\%$ Check $x \operatorname{dot}=0$

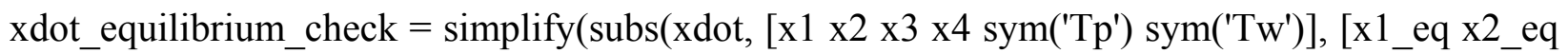
x3_eq x4_eq Tp_eq Tw_eq]))

\%\%\%\%\%\%\%\%\%\%\%\%\%\% LINEARIZED SS MATRICES \%\%\%\%\%\%\%\%\%\%\%\%\%\%\%

A $=\operatorname{subs}\left(\right.$ A_temp, $\left[\mathrm{x} 1 \times 2 \times 3 \times 4 \operatorname{sym}\left(' T p^{\prime}\right) \operatorname{sym}(' T w ')\right],\left[x 1 \_e q x 2\right.$ eq x3_eq x4_eq Tp_eq Tw_eq]); B_temp1 $=$ subs(B_temp, [x1 x2 x3 x4 sym('Tp') sym('Tw')], [x1_eq x2_eq x3_eq x4_eq Tp_eq Tw_eq]);

B = B_temp1(:,1); \%only first column

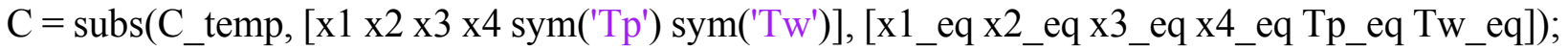


D_temp1 $=\operatorname{subs}($ D_temp, $[\mathrm{x} 1 \mathrm{x} 2 \times 3 \times$ x4 sym('Tp') sym('Tw')], [x1_eq x2_eq x3_eq x4_eq Tp_eq Tw_eq]);

$\mathrm{D}=\mathrm{D} \_$temp1(:,1); \%Only first column

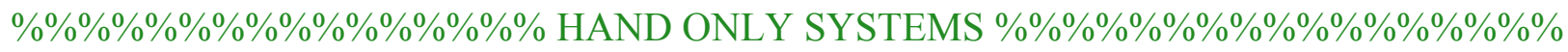

if strcmp(Rotation, 'FE')

$$
\begin{aligned}
& \text { A_h }=\left[\begin{array}{lllllllll}
0 & 0 & 1 & 0 & 0 & 0 & 0 & 0 & 0 ;
\end{array}-\left(\mathrm{kw}-\mathrm{g}^{*} \mathrm{mw}^{*} \mathrm{yw}\right) /\left(\mathrm{mw}^{*} \mathrm{xw}^{\wedge} 2+\mathrm{mw}^{*} \mathrm{yw}^{\wedge} 2+\mathrm{Iwz}\right) 0-\mathrm{Dw} /\left(\mathrm{mw}^{*} \mathrm{xw}^{\wedge} 2\right.\right. \\
& \left.+\mathrm{mw}^{*} \mathrm{yw}^{\wedge} 2+\mathrm{Iwz}\right) \text { 0; } 000000 \text {; } \\
& \text { B_h }=\left[0 ; 0 ; 1 /\left(\mathrm{mw}^{*} \mathrm{xw}^{\wedge} 2+\mathrm{mw}^{*} \mathrm{yw}^{\wedge} 2+\mathrm{Iwz}\right) ; 0\right] \\
& \mathrm{C}_{-} \mathrm{h}=\left[\begin{array}{llll}
1 & 0 & 0 & 0
\end{array}\right] \text {; } \\
& \text { D_h }=0 \text {; }
\end{aligned}
$$

elseif strcmp(Rotation, 'RUD')

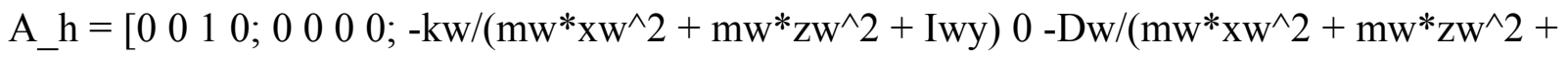
Iwy) 0; 000 llll;

B_h $=\left[0 ; 0 ; 1 /\left(\mathrm{mw}^{*} \mathrm{xw}^{\wedge} 2+\mathrm{mw}^{*} \mathrm{zw}^{\wedge} 2+\mathrm{Iwy}\right) ; 0\right]$

$\mathrm{C} \_\mathrm{h}=\left[\begin{array}{llll}1 & 0 & 0 & 0\end{array}\right]$

D_h $=0$;

elseif strcmp(Rotation, 'PS')

A_h $=\left[\begin{array}{lllllllll}0 & 0 & 1 & 0 & 0 & 0 & 0 & 0 & 0\end{array} ;-\left(\mathrm{kw}-\mathrm{g}^{*} \mathrm{mw}^{*} \mathrm{yw}\right) /\left(\mathrm{mw}^{*} \mathrm{yw}^{\wedge} 2+\mathrm{mw}^{*} \mathrm{zw}^{\wedge} 2+\mathrm{Iwx}\right) 0-\mathrm{Dw} /\left(\mathrm{mw}^{*} \mathrm{yw}^{\wedge} 2\right.\right.$ $\left.+\mathrm{mw}^{*} \mathrm{Zw}^{\wedge} 2+\operatorname{Iwx}\right)$ 0; 000000 ;

B_h $=\left[0 ; 0 ; 1 /\left(\mathrm{mw}^{*} \mathrm{yw}^{\wedge} 2+\mathrm{mw}^{*} \mathrm{zw}^{\wedge} 2+\mathrm{Iwx}\right) ; 0\right]$

C_h $=\left[\begin{array}{llll}1 & 0 & 0 & 0\end{array}\right]$;

D_h $=0$;

end 


\title{
D.1 Description
}

Complete_1DOF.m calculates the SS matrices of the model and Sensitivity.m is used to substitute parameters into these SS matrices. Sensitivity.m was used to generate figures 3.3 to 3.7. When performing a sensitivity analysis for a given parameter, all other parameters use their default values. The parameter of interest is then varied from its default value to each of its variation values (Table 2-1) and the resulting bode plots for each variation are plotted on the same plot. Note that the code for Sensitivity.m presented in the next section is only partial. Only the code for the sensitivity analysis of the first parameter, precession damping, is included for the sake of space.

\author{
D.2 Sensitivity.m (partial) \\ $\%$ SET BODE PLOT SETTINGS \\ opts = bodeoptions('cstprefs'); \\ opts.FreqUnits = 'Hz'; \\ opts.FreqScale = 'linear'; \\ opts.MagUnits = 'abs'; \\ opts.Xlim $=[0.1,12]$; \\ gender $=$ 'default'
}


\%Parameters updated NOV 28th, 2017 (last update: update rg)

\%\%\%\%\%\%\%\%\%\% PUT IN PARAMETER VALUES \%\%\%\%\%\%\%\%\%\%\%\%\%\%\%\%

\%\%\%\%\%\%\%\%\%\%\%\%\%\% Gyroscope Parameters \%\%\%\%\%\%\%\%\%\%\%\%\%\%\%\%\%\%\%

$\mathrm{xg}=0.04 ; \%(\mathrm{~m})$ distance to COM of the gyro from the wrist

yg $=0.03 ; \quad \%(m)$ distance to COM of the gyro above hand

$\mathrm{zg}=0$

$\%$ yg $=0.02$

$\% \mathrm{zg}=0.1$

$\operatorname{lam}=10000 ; \% \mathrm{rpm}$

$\mathrm{kp}=5$;

$\mathrm{Dp}=0.1$

lam $=1 \mathrm{am} * 2 * \mathrm{pi} / 60$

\%\%\%\%\%\%\%\%\%\%\%\%\% Gyroscope motor Parameters (Body 1) \%\%\%\%\%\%\%\%\%\%\%\%\%

$\mathrm{Rm}=0.01 ; \%(\mathrm{~m})$ radius of gyro flywheel

$\mathrm{Lm}=0.03 ; \%(\mathrm{~m})$ length of flywheel

densitym $=3750 ; \% \mathrm{~kg} / \mathrm{m}^{\wedge} 3$

$\mathrm{rm}=-0.01$;

$\mathrm{mm}=\left(\mathrm{pi}^{*} \mathrm{Rm}^{\wedge} 2 * \mathrm{Lm}\right) *$ densitym $\%(\mathrm{~kg})$ mass of the gyro motor

$\operatorname{Imx}=0.25 * \mathrm{~mm} * \mathrm{Rm}^{\wedge} 2+(1 / 12) * \mathrm{~mm} * \mathrm{Lm}^{\wedge} 2 ; \quad \%\left(\mathrm{~kg}^{*} \mathrm{~m}^{\wedge} 2\right)$

$\operatorname{Imy}=0.25 * \mathrm{~mm} * \mathrm{Rm}^{\wedge} 2+(1 / 12) * \mathrm{~mm} * \mathrm{Lm}^{\wedge} 2 ; \quad \%\left(\mathrm{~kg}^{*} \mathrm{~m}^{\wedge} 2\right)$

$\operatorname{Imz}=1 / 2 * m m^{*} \mathrm{Rm}^{\wedge} 2 ; \%\left(\mathrm{~kg}^{*} \mathrm{~m}^{\wedge} 2\right)$ inertia of the flywheel assuming it is a cylinder

\%\%\%\%\%\%\%\%\%\%\% Gyro Flywheel Parameters (Body 2)\%\%\%\%\% \%\%\% \%\%\%\%\%

$\mathrm{Rg}=0.03 ; \%(\mathrm{~m})$ radius of gyro flywheel 
$\mathrm{L}=0.02 ; \%(\mathrm{~m})$ length of flywheel

density $=7850 ; \% \mathrm{~kg} / \mathrm{m}^{\wedge} 3$

$\operatorname{rg}=-0.01$

$\% \mathrm{rg}=0$

\%Model as a Cylinder

$\mathrm{mg}=\left(\mathrm{pi}^{*} \mathrm{Rg}^{\wedge} 2 * \mathrm{~L}\right) *$ density $\%(\mathrm{~kg})$ mass of the gyro flywheel

$\mathrm{Ix}=0.25 * \mathrm{mg}^{*} \operatorname{Rg}^{\wedge} 2+(1 / 12) * \mathrm{mg}^{*} \mathrm{~L}^{\wedge} 2 ; \quad \%\left(\mathrm{~kg}^{*} \mathrm{~m}^{\wedge} 2\right)$

$\mathrm{Iy}=\mathrm{Ix}$

$\mathrm{Iz}=1 / 2 * \mathrm{mg}^{*} \mathrm{Rg}^{\wedge} 2 ; \%\left(\mathrm{~kg}^{*} \mathrm{~m}^{\wedge} 2\right)$

\%\%\%\%\%\%\%\%\%\%\%\%\%\%\%\%\% Hand Parameters (Body 3) \%\%\%\%\%\%\%\%\%\%\%\%\%\%\%\%

if strcmp(gender, 'male')

$\mathrm{xw}=0.068 ; \%(\mathrm{~m})$ distance to the $\mathrm{COM}$ of hand from the wrist

$\mathrm{yw}=0$

$\mathrm{zW}=0$

$\mathrm{Iwx}=0.0018 ; \% \mathrm{~kg}^{*} \mathrm{~m}^{\wedge} 2$ represents PS

Iwy $=0.0013 ; \% \mathrm{~kg}^{*} \mathrm{~m}^{\wedge} 2$ represents RUD

$\mathrm{Iwz}=0.0009 ; \% \mathrm{~kg}^{*} \mathrm{~m}^{\wedge} 2$ represents FE about $\mathrm{COM}$

$\mathrm{mw}=.45 ; \%(\mathrm{~kg})$ mass of the hand

elseif strcmp(gender, 'female')

$\mathrm{xw}=0.058 ; \%(\mathrm{~m})$ distance to the $\mathrm{COM}$ of hand from the wrist

$\mathrm{yw}=0$

$\mathrm{zW}=0$

Iwx $=0.0008 ; \% \mathrm{~kg}^{*} \mathrm{~m}^{\wedge} 2$ represents PS 


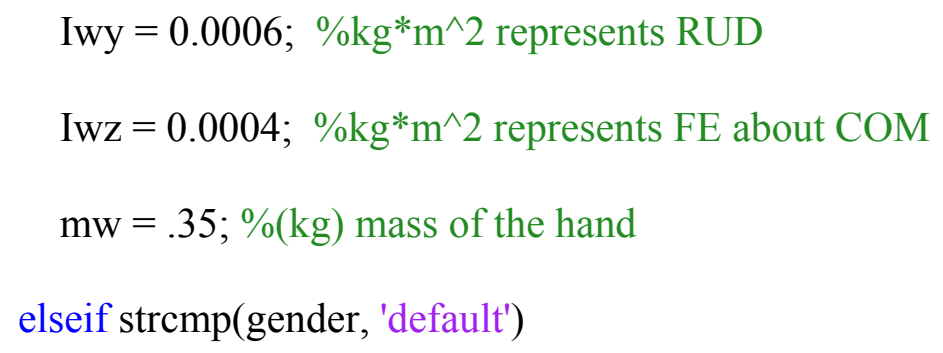


$\% \% \% \% \% \% \% \% \% \% \% \% \% \% \%$ Remaining $\% \% \% \% \% \% \% \% \% \% \% \% \% \%$

$\mathrm{g}=9.8 ; \% \mathrm{~m} / \mathrm{s}^{\wedge} 2$

\%\%\%\%\%\%\%\%\%\%\%\%\% HAND ONLY SYSTEMS \%\%\%\%\%\%\%\%\%\%\%\%\%\%\%\%\%\%\%\%

A_hand = double(subs(A_h, [sym('lam') sym('kp') sym('Dp') sym('mg') sym('Ix') sym('Iy') sym('Iz') $\operatorname{sym}\left({ }^{\prime} g^{\prime}\right) \operatorname{sym}(' y g$ ') sym('zg') sym('rg') sym('mm') sym('Imx') sym('Imy') sym('Imz') sym('rm') sym('mw') sym('xw') sym('yw') sym('zw') sym('kw') sym('Iwx') sym('Iwy') sym('Iwz') sym('Dw') sym('g')],[lam kp Dp mg Ix Iy Iz xg yg zg rg mm Imx Imy Imz rm mw xw yw zw kw Iwx Iwy Iwz Dw g]));

B_hand = double(subs(B_h, [sym('lam') sym('kp') sym('Dp') sym('mg') sym('Ix') sym('Iy') sym('Iz') $\operatorname{sym}\left({ }^{\prime} g^{\prime}\right) \operatorname{sym}(' y g$ ') $\operatorname{sym}(' z g ') \operatorname{sym}(' r g ')$ sym('mm') sym('Imx') sym('Imy') sym('Imz') sym('rm') sym('mw') sym('xw') sym('yw') sym('zw') sym('kw') sym('Iwx') sym('Iwy') sym('Iwz') sym('Dw') sym('g')],[lam kp Dp mg Ix Iy Iz xg yg zg rg mm Imx Imy Imz rm mw xw yw zw kw Iwx Iwy Iwz Dw g]));

C_hand = double(subs(C_h, [sym('lam') sym('kp') sym('Dp') sym('mg') sym('Ix') sym('Iy') sym('Iz') sym('xg') sym('yg') sym('zg') sym('rg') sym('mm') sym('Imx') sym('Imy') sym('Imz') sym('rm') sym('mw') sym('xw') sym('yw') sym('zw') sym('kw') sym('Iwx') sym('Iwy') sym('Iwz') sym('Dw') sym('g')],[lam kp Dp mg Ix Iy Iz xg yg zg rg mm Imx Imy Imz rm mw xw yw zw kw Iwx Iwy Iwz Dw g]));

D_hand = double(subs(D_h, [sym('lam') sym('kp') sym('Dp') sym('mg') sym('Ix') sym('Iy') sym('Iz') sym('xg') sym('yg') sym('zg') sym('rg') sym('mm') sym('Imx') sym('Imy') sym('Imz') sym('rm') sym('mw') sym('xw') sym('yw') sym('zw') sym('kw') sym('Iwx') sym('Iwy') sym('Iwz') sym('Dw') sym('g')],[lam kp Dp mg Ix Iy Iz xg yg zg rg mm Imx Imy Imz rm mw xw yw zw kw Iwx Iwy Iwz Dw g])); 
sys_hand $=$ ss(A_hand,B_hand,C_hand,D_hand);

\%\%\%\%\%\%\%\%\%\%\%\%\%\%\%\%\%\%\% Default SYSTEM \%\%\%\%\%\%\%\%\%\%\%\%\%\%\%\%

A_def $=$ double(subs(A, [sym('lam') sym('kp') sym('Dp') sym('mg') sym('Ix') sym('Iy') sym('Iz') $\operatorname{sym}(' x g$ ') $\operatorname{sym}(' y g$ ') sym('zg') sym('rg') sym('mm') sym('Imx') sym('Imy') sym('Imz') sym('rm') sym('mw') sym('xw') sym('yw') sym('zw') sym('kw') sym('Iwx') sym('Iwy') sym('Iwz') sym('Dw') sym('g')],[lam kp Dp mg Ix Iy Iz xg yg zg rg mm Imx Imy Imz rm mw xw yw zw kw Iwx Iwy Iwz Dw g]));

B_def $=$ double(subs(B, [sym('lam') sym('kp') sym('Dp') sym('mg') sym('Ix') sym('Iy') sym('Iz') $\operatorname{sym}($ 'xg') sym('yg') sym('zg') sym('rg') sym('mm') sym('Imx') sym('Imy') sym('Imz') sym('rm') sym('mw') sym('xw') sym('yw') sym('zw') sym('kw') sym('Iwx') sym('Iwy') sym('Iwz') sym('Dw') sym('g')],[lam kp Dp mg Ix Iy Iz xg yg zg rg mm Imx Imy Imz rm mw xw yw zw kw Iwx Iwy Iwz Dw g]));

C_def $=$ C;

D_def = D;

sys_def $=$ ss(A_def,B_def,C_def,D_def);

$\% \% \% \% \% \% \% \% \% \% \% \%$ Gyro Off SYSTEM \%\%\%\%\%\%\%\%\%\%\%\%\%\%\%\%

A_off $=$ double(subs(A, [sym('lam') sym('kp') sym('Dp') sym('mg') sym('Ix') sym('Iy') sym('Iz') sym('xg') sym('yg') sym('zg') sym('rg') sym('mm') sym('Imx') sym('Imy') sym('Imz') sym('rm') sym('mw') sym('xw') sym('yw') sym('zw') sym('kw') sym('Iwx') sym('Iwy') sym('Iwz') sym('Dw') sym('g')],[0 kp Dp mg Ix Iy Iz xg yg zg rg mm Imx Imy Imz rm mw xw yw zw kw Iwx Iwy Iwz Dw g]));

B_off = double(subs(B, [sym('lam') sym('kp') sym('Dp') sym('mg') sym('Ix') sym('Iy') sym('Iz') sym('xg') sym('yg') sym('zg') sym('rg') sym('mm') sym('Imx') sym('Imy') sym('Imz') sym('rm') 
sym('mw') sym('xw') sym('yw') sym('zw') sym('kw') sym('Iwx') sym('Iwy') sym('Iwz') sym('Dw') sym('g')],[0 kp Dp mg Ix Iy Iz xg yg zg rg mm Imx Imy Imz rm mw xw yw zw kw Iwx Iwy Iwz Dw g]));

C_off $=$ C;

D_off $=$ D;

sys_off = ss(A_off,B_off,C_off,D_off);

figure(1)

opts.Title.String = 'Default Parameters';

bodeplot(sys_def,sys_off,sys_hand, opts)

legend('Default', 'Gyro Off,'Hand')

hold on

[def_magpeak,def_fpeak]= getPeakGain(sys_def);

def_fpeak $=(1 /(2 *$ pi $)) *$ def_fpeak

def_magpeak

def_bw $=(1 /(2 *$ pi $)) *$ bandwidth(sys_def $)$

[off_magpeak,off_fpeak] = getPeakGain(sys_off);

off_fpeak $=(1 /(2 *$ pi $)) *$ off_fpeak

off_magpeak

off_bw $=(1 /(2 *$ pi $)) *$ bandwidth(sys_off)

[hand_magpeak,hand_fpeak] = getPeakGain(sys_hand);

hand_fpeak $=(1 /(2 * \mathrm{pi})))^{*}$ hand_fpeak

hand_magpeak 
hand_bw $=(1 /(2 *$ pi) $) *$ bandwidth(sys_hand)

\%\%\%\%\%\%\%\%\%\%\%\%\%\% BEGIN SENSITIVITY ANALYSIS BELOW \%\%\%\%\%\%\%\%\%\%\%

\%\%\%\%\%\%\%\%\%\%\%\%\%\%\%\%\%\% Gyro Parameters \%\%\%\%\%\%\%\%\%\%\%\%\%\%\%

\%\%\%\%\%\%\%\%\%\%\%\%\%\%\%\%\%\%\% Dp \%\%\%\%\%\%\%\%\%\%\%\%\%\%\%\%\%\%\%\%\%

Dp1 $=0.05$;

A_1 = double(subs(A, [sym('lam') sym('kp') sym('Dp') sym('mg') sym('Ix') sym('Iy') sym('Iz') sym('xg') sym('yg') sym('zg') sym('rg') sym('mm') sym('Imx') sym('Imy') sym('Imz') sym('rm') sym('mw') sym('xw') sym('yw') sym('zw') sym('kw') sym('Iwx') sym('Iwy') sym('Iwz') sym('Dw') sym('g')],[lam kp Dp1 mg Ix Iy Iz xg yg zg rg mm Imx Imy Imz rm mw xw yw zw kw Iwx Iwy Iwz Dw g]));

B_1 = double(subs(B, [sym('lam') sym('kp') sym('Dp') sym('mg') sym('Ix') sym('Iy') sym('Iz') $\operatorname{sym}($ 'xg') sym('yg') sym('zg') sym('rg') sym('mm') sym('Imx') sym('Imy') sym('Imz') sym('rm') $\operatorname{sym}(' m w ') \operatorname{sym}(' x w ') \operatorname{sym}(' y w ') \operatorname{sym}(' z w ') \operatorname{sym}(' k w ')$ sym('Iwx') sym('Iwy') sym('Iwz') sym('Dw') sym('g')],[lam kp Dp1 mg Ix Iy Iz xg yg zg rg mm Imx Imy Imz rm mw xw yw zw kw Iwx Iwy Iwz Dw g]));

C_1 $=\mathrm{C}$;

D_1 = D;

sys_1 $=$ ss(A_1,B_1,C_1,D_1);

$\mathrm{Dp} 2=0.2 ;$

A_2 $=$ double(subs(A, [sym('lam') sym('kp') sym('Dp') sym('mg') sym('Ix') sym('Iy') sym('Iz') sym('xg') sym('yg') sym('zg') sym('rg') sym('mm') sym('Imx') sym('Imy') sym('Imz') sym('rm') $\operatorname{sym}(' m w ') \operatorname{sym}(' x w ') \operatorname{sym}(' y w ') \operatorname{sym}(' z w ') \operatorname{sym}(' k w ')$ sym('Iwx') sym('Iwy') sym('Iwz') sym('Dw') sym('g')],[lam kp Dp2 mg Ix Iy Iz xg yg zg rg mm Imx Imy Imz rm mw xw yw zw kw Iwx Iwy 
Iwz Dw g]));

B_2 = double( $\operatorname{subs}($ B, [sym('lam') sym('kp') sym('Dp') sym('mg') sym('Ix') sym('Iy') sym('Iz') sym('xg') sym('yg') sym('zg') sym('rg') sym('mm') sym('Imx') sym('Imy') sym('Imz') sym('rm') sym('mw') sym('xw') sym('yw') sym('zw') sym('kw') sym('Iwx') sym('Iwy') sym('Iwz') sym('Dw') sym('g')],[lam kp Dp2 mg Ix Iy Iz xg yg zg rg mm Imx Imy Imz rm mw xw yw zw kw Iwx Iwy Iwz Dw g]));

C_2 $=\mathrm{C}$;

D_2 = D;

sys_2 =ss(A_2,B_2,C_2,D_2);

$\mathrm{Dp} 3=1$

A_3 = double(subs(A, [sym('lam') sym('kp') sym('Dp') sym('mg') sym('Ix') sym('Iy') sym('Iz') sym('xg') sym('yg') sym('zg') sym('rg') sym('mm') sym('Imx') sym('Imy') sym('Imz') sym('rm') sym('mw') sym('xw') sym('yw') sym('zw') sym('kw') sym('Iwx') sym('Iwy') sym('Iwz') sym('Dw') sym('g')],[lam kp Dp3 mg Ix Iy Iz xg yg zg rg mm Imx Imy Imz rm mw xw yw zw kw Iwx Iwy Iwz Dw g]));

B_3 = double(subs(B, [sym('lam') sym('kp') sym('Dp') sym('mg') sym('Ix') sym('Iy') sym('Iz')

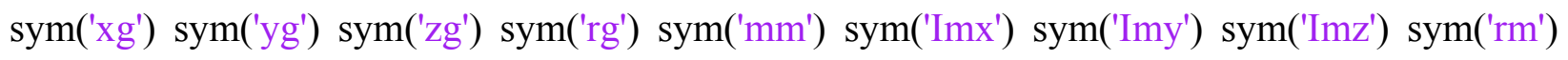
$\operatorname{sym}(' m w ') \operatorname{sym}(' x w ') \operatorname{sym}(' y w ') \operatorname{sym}(' z w ') \operatorname{sym}(' k w ') \operatorname{sym}(' I w x ') \operatorname{sym}(' I w y ') \operatorname{sym}(' I w z ') \operatorname{sym}(' D w ')$ sym('g')],[lam kp Dp3 mg Ix Iy Iz xg yg zg rg mm Imx Imy Imz rm mw xw yw zw kw Iwx Iwy Iwz Dw g]));

C_3 $=\mathrm{C}$;

D_3 = D;

sys_3 = ss(A_3,B_3,C_3,D_3); 
Dp4 = 5;

A_4 = double(subs(A, [sym('lam') sym('kp') sym('Dp') sym('mg') sym('Ix') sym('Iy') sym('Iz') sym('xg') sym('yg') sym('zg') sym('rg') sym('mm') sym('Imx') sym('Imy') sym('Imz') sym('rm') sym('mw') sym('xw') sym('yw') sym('zw') sym('kw') sym('Iwx') sym('Iwy') sym('Iwz') sym('Dw') sym('g')],[lam kp Dp4 mg Ix Iy Iz xg yg zg rg mm Imx Imy Imz rm mw xw yw zw kw Iwx Iwy Iwz Dw g]));

B_4 = double(subs(B, [sym('lam') sym('kp') sym('Dp') sym('mg') sym('Ix') sym('Iy') sym('Iz') sym('xg') sym('yg') sym('zg') sym('rg') sym('mm') sym('Imx') sym('Imy') sym('Imz') sym('rm') sym('mw') sym('xw') sym('yw') sym('zw') sym('kw') sym('Iwx') sym('Iwy') sym('Iwz') sym('Dw') sym('g')],[lam kp Dp4 mg Ix Iy Iz xg yg zg rg mm Imx Imy Imz rm mw xw yw zw kw Iwx Iwy Iwz Dw g]));

C_4 $=$ C;

D_4 = D;

sys_4 $=\operatorname{ss}($ A_4,B_4,C_4,D_4);

figure(2)

suptitle('General Gyroscope Parameters');

$\operatorname{subplot}(3,2,4)$

opts.Title.String = 'Change in Dp';

bodeplot(sys_1,sys_def, sys_2,sys_3,sys_4,sys_hand,sys_off, opts)

legend('Dp = 0.05','Dp = 0.1', 'Dp = 0.2', 'Dp = 1', 'Dp = 5', 'Hand','gyro off,'Location','Best') 


\section{E.1 Description}

LINvsNL_Main.m uses ode45.m together with LINvsNL.m to solve the nonlinear EOMs. The nonlinear EOMS are located in LINvsNL.m. The code for LINvsNL.m is only partially shown because it would otherwise be too long. The nonlinear EOMs, called xdot, are obtained from running complete_1DOF.m. To allow for xdot to be pasted into LINvsNL.m, xdot needs to first be modified. This is because $\mathrm{x} 1, \mathrm{x} 2, \mathrm{x} 3$, and $\mathrm{x} 4$ must be $\mathrm{x}(1), \mathrm{x}(2), \mathrm{x}(3)$, and $\mathrm{x}(4)$ respectively in LINvsNL.m. This change can be implemented via the following code:

$$
\begin{aligned}
& \operatorname{syms} x(a) x(b) x(c) x(d) \\
& x \operatorname{xdot} 2=\operatorname{subs}(x \operatorname{dot},[x 1 \times 2 \times 3 \times 4],[x(a) x(b) x(c) x(d)]) ; \\
& x \operatorname{xdot}=\operatorname{subs}\left(x \operatorname{dot} 2,\left[\begin{array}{lll}
a & b & c
\end{array}\right],\left[\begin{array}{llll}
1 & 2 & 3 & 4
\end{array}\right]\right) ;
\end{aligned}
$$

Complete_1DOF.m needs to be run for the 12 cardinal configurations and then the above code needs to be run to modify xdot. This new xdot should be pasted into LINvsNL for all 12 cardinal configurations. To use LINvsNL_Main.m, the first step is to run Complete_1DOF.m with the desired wrist DOF and gyroscope orientation inputs to obtain the SS matrices. The next step is to input the desired parameter values into LINvsNL_Main.m. LINvsNL_Main.m substitutes these parameters into the SS matrices. Then ode45.m is used with LINvsNL.m to solve the nonlinear EOMs. Then the linear and nonlinear results are compared and plotted together to determine the 
effectiveness of the linearization. The steady-state error is also calculated and plotted. These two functions were used to generate Figure 3-1 and to obtain the information to create Figure 3-2.

\section{E.2 LINvsNL_Main.m}

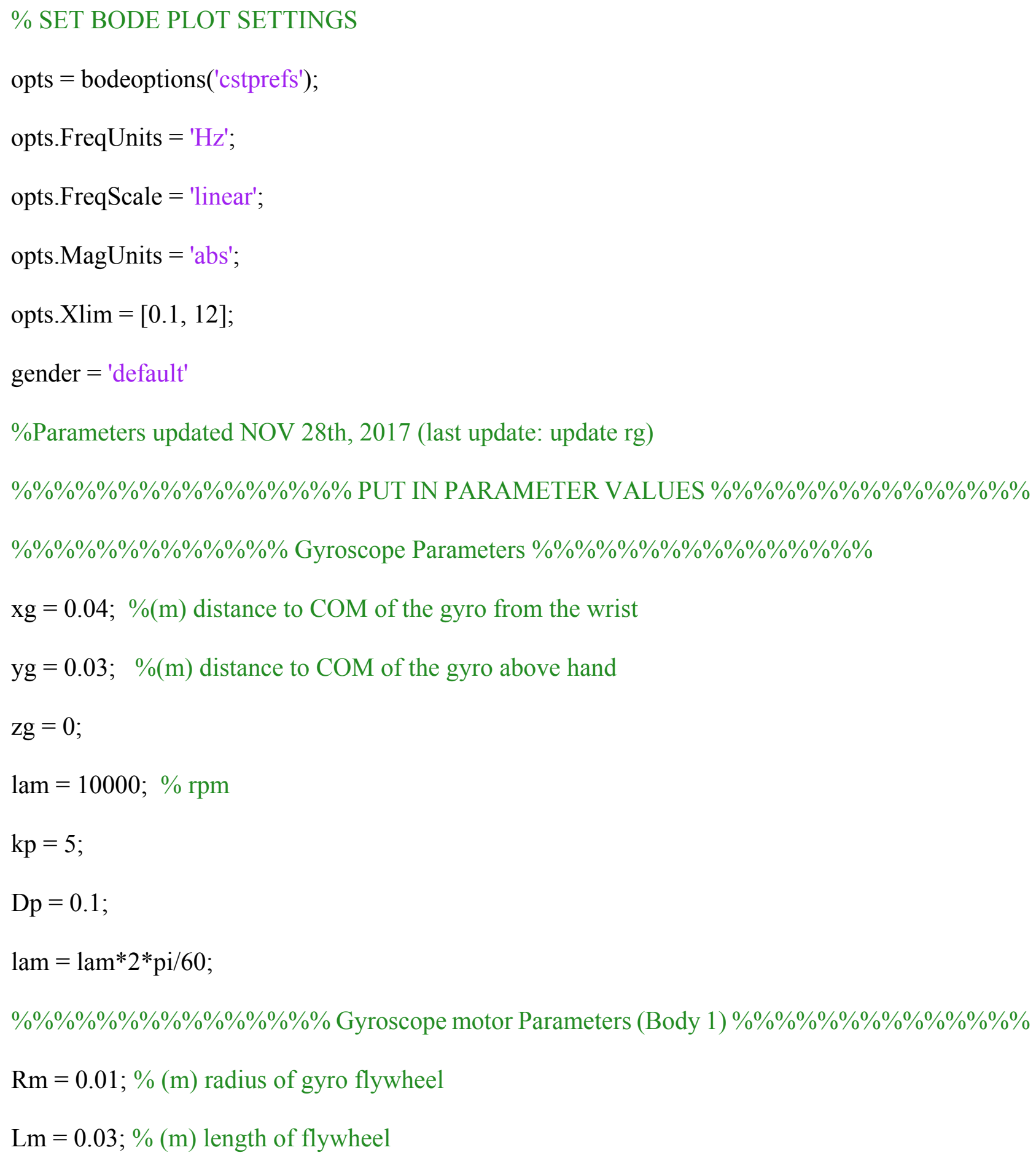


densitym $=3750 ; \% \mathrm{~kg} / \mathrm{m}^{\wedge} 3$

$\mathrm{rm}=-0.01$

$\mathrm{mm}=\left(\mathrm{pi}^{*} \mathrm{Rm}^{\wedge} 2 * \mathrm{Lm}\right) *$ densitym $\%(\mathrm{~kg})$ mass of the gyro motor

$\operatorname{Imx}=0.25 * m m * \mathrm{Rm}^{\wedge} 2+(1 / 12) * m m * \mathrm{Lm}^{\wedge} 2 ; \quad \%\left(\mathrm{~kg}^{*} \mathrm{~m}^{\wedge} 2\right)$

$\operatorname{Imy}=0.25^{*} \mathrm{~mm}^{*} \mathrm{Rm}^{\wedge} 2+(1 / 12)^{*} \mathrm{~mm} * \mathrm{Lm}^{\wedge} 2 ; \quad \%\left(\mathrm{~kg}^{*} \mathrm{~m}^{\wedge} 2\right)$

$\operatorname{Imz}=1 / 2 * \mathrm{~mm}^{*} \mathrm{Rm}^{\wedge} 2 ; \%\left(\mathrm{~kg}^{*} \mathrm{~m}^{\wedge} 2\right)$ inertia of the flywheel assuming it is a cylinder

\%\%\%\%\%\%\%\%\%\%\%\%\%\% Gyro Flywheel Parameters (Body 2)\%\%\%\%\%\%\%\%\%\%\%\%\%\%\%\%

$\mathrm{Rg}=0.03 ; \%(\mathrm{~m})$ radius of gyro flywheel

$\mathrm{L}=0.02 ; \%(\mathrm{~m})$ length of flywheel

density $=7850 ; \% \mathrm{~kg} / \mathrm{m}^{\wedge} 3$

$\mathrm{rg}=-0.01$

$\%$ Model as a Cylinder

$\mathrm{mg}=\left(\mathrm{pi}^{*} \mathrm{Rg}^{\wedge} 2 * \mathrm{~L}\right) *$ density $\%(\mathrm{~kg})$ mass of the gyro flywheel

$\mathrm{Ix}=0.25^{*} \mathrm{mg}^{*} \mathrm{Rg}^{\wedge} 2+(1 / 12) * \mathrm{mg}^{*} \mathrm{~L}^{\wedge} 2 ; \quad \%\left(\mathrm{~kg}^{*} \mathrm{~m}^{\wedge} 2\right)$

$\mathrm{Iy}=\mathrm{Ix}$

$\mathrm{Iz}=1 / 2 * \mathrm{mg}^{*} \operatorname{Rg}^{\wedge} 2 ; \%\left(\mathrm{~kg}^{*} \mathrm{~m}^{\wedge} 2\right)$

\%\%\%\%\%\%\%\%\%\%\%\%\%\%\% Hand Parameters (Body 3) \%\%\%\%\%\%\%\%\%\%\%\%\%\%\%\%\%\%

if strcmp(gender, 'male')

$$
\begin{aligned}
& \mathrm{xw}=0.068 ; \%(\mathrm{~m}) \text { distance to the COM of hand from the wrist } \\
& \mathrm{yw}=0 ; \\
& \mathrm{zW}=0 ;
\end{aligned}
$$




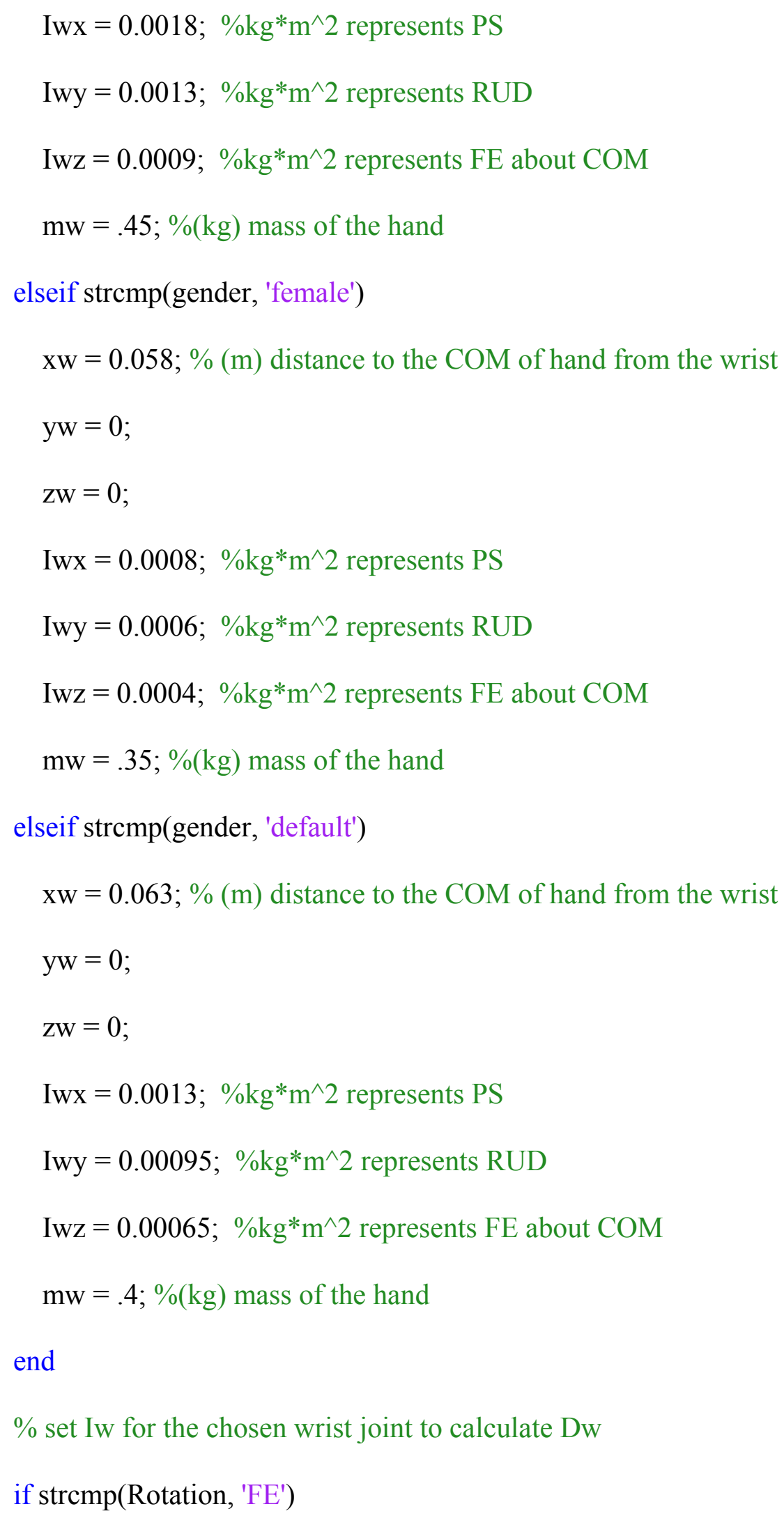




$$
\mathrm{Iw} 1=\mathrm{Iwz}+\mathrm{mw}^{*} \mathrm{xw}^{\wedge} 2 ;
$$

elseif strcmp(Rotation, 'RUD')

$\mathrm{Iw} 1=\mathrm{Iwy}+\mathrm{mw}^{*} \mathrm{xw}^{\wedge} 2$

elseif strcmp(Rotation, 'PS')

$\operatorname{Iw} 1=\operatorname{Iwx}$

end

$\mathrm{kw}=1 ; \% \mathrm{Nm} / \mathrm{rad}$ This is for passive stiffness, dynamic can bring up to 10

damp_ratio $=.375 ; \% 0.25$ to 0.5

$\mathrm{Dw}=2 *$ damp_ratio*sqrt( $\mathrm{kw} * \mathrm{Iw} 1)$;

\%\%\%\%\%\%\%\%\%\%\%\%\%\%\%\%\%\%\% Remaining \%\%\%\%\%\%\%\%\%\%\%\%\%\%\%\%\%\%\%\%\%\%

$\mathrm{g}=9.8 ; \% \mathrm{~m} / \mathrm{s}^{\wedge} 2$

\%\%\%\%\%\%\%\%\%\%\%\%\%\%\%\% HAND ONLY SYSTEMS \%\%\%\%\%\%\%\%\%\%\%\%\%\%\%\%\%\%

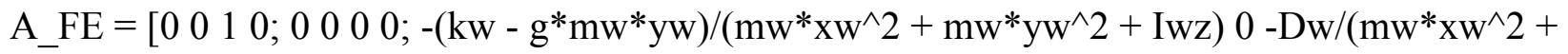
$\left.\mathrm{mw}^{*} \mathrm{yw}^{\wedge} 2+\mathrm{Iwz}\right)$ 0; $\left.\begin{array}{lllll}0 & 0 & 0 & 0\end{array}\right]$

B_FE $=\left[0 ; 0 ; 1 /\left(\mathrm{mw}^{*} \mathrm{xw}^{\wedge} 2+\mathrm{mw}^{*} \mathrm{yw}^{\wedge} 2+\mathrm{Iwz}\right) ; 0\right] ;$

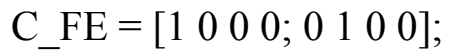

D_FE $=[0 ; 0]$;

sys_FE $=$ ss(A_FE, B_FE, C_FE, D_FE);

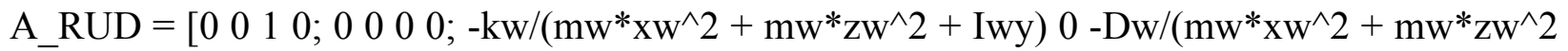

+ Iwy) 0; $\left.\begin{array}{lllll}0 & 0 & 0\end{array}\right]$

B_RUD $=\left[0 ; 0 ; 1 /\left(\mathrm{mw}^{*} \mathrm{xw}^{\wedge} 2+\mathrm{mw}^{*} \mathrm{zw}^{\wedge} 2+\right.\right.$ Iwy $\left.) ; 0\right] ;$

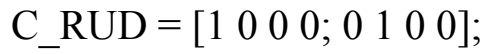


D_RUD $=[0 ; 0]$

sys_RUD $=\operatorname{ss}($ A_RUD, B_RUD, C_RUD, D_RUD);

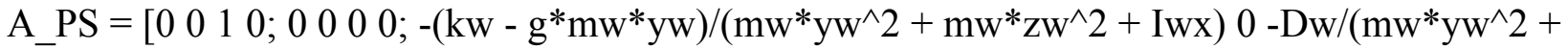
$\left.\left.\mathrm{mw}^{*} \mathrm{Zw}^{\wedge} 2+\mathrm{Iwx}\right) 0 ; 0 \begin{array}{llll}0 & 0 & 0 & 0\end{array}\right]$

B_PS $=\left[0 ; 0 ; 1 /\left(\mathrm{mw}^{*} \mathrm{yw}^{\wedge} 2+\mathrm{mw}^{*} \mathrm{zw}^{\wedge} 2+\mathrm{Iwx}\right) ; 0\right]$

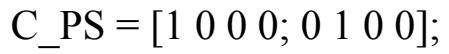

D_PS $=[0 ; 0]$

sys_PS $=$ ss(A_PS, B_PS, C_PS, D_PS $)$;

$\%$ Set current wrist joint automatically

if strcmp(Rotation, 'FE')

sys_hand = sys_FE;

elseif strcmp(Rotation, 'RUD')

sys_hand = sys_RUD;

elseif strcmp(Rotation, 'PS')

sys_hand $=$ sys_PS;

end

\%\%\%\%\%\%\%\%\%\%\%\%\%\%\%\%\%\% Default SYSTEM \%\%\%\%\%\%\%\%\%\%\%\%\%\%\%\%\%\%\%

A_def $=$ double(subs(A, [sym('lam') sym('kp') sym('Dp') sym('mg') sym('Ix') sym('Iy') sym('Iz') sym('xg') sym('yg') sym('zg') sym('rg') sym('mm') sym('Imx') sym('Imy') sym('Imz') sym('rm') sym('mw') sym('xw') sym('yw') sym('zw') sym('kw') sym('Iwx') sym('Iwy') sym('Iwz') sym('Dw') sym('g')],[lam kp Dp mg Ix Iy Iz xg yg zg rg mm Imx Imy Imz rm mw xw yw zw kw Iwx Iwy Iwz Dw g])); 
B_def $=$ double(subs(B, [sym('lam') sym('kp') sym('Dp') sym('mg') sym('Ix') sym('Iy') sym('Iz') $\operatorname{sym}(' x g$ ') $\operatorname{sym}(' y g$ ') sym('zg') sym('rg') sym('mm') sym('Imx') sym('Imy') sym('Imz') sym('rm') sym('mw') sym('xw') sym('yw') sym('zw') sym('kw') sym('Iwx') sym('Iwy') sym('Iwz') sym('Dw') sym('g')],[lam kp Dp mg Ix Iy Iz xg yg zg rg mm Imx Imy Imz rm mw xw yw zw kw Iwx Iwy Iwz Dw g]));

C_def $=$ double(subs(C, [sym('lam') sym('kp') sym('Dp') sym('mg') sym('Ix') sym('Iy') sym('Iz') sym('xg') sym('yg') sym('zg') sym('rg') sym('mm') sym('Imx') sym('Imy') sym('Imz') sym('rm') sym('mw') sym('xw') sym('yw') sym('zw') sym('kw') sym('Iwx') sym('Iwy') sym('Iwz') sym('Dw') sym('g')],[lam kp Dp mg Ix Iy Iz xg yg zg rg mm Imx Imy Imz rm mw xw yw zw kw Iwx Iwy Iwz Dw g]));

D_def = double(subs(D, [sym('lam') sym('kp') sym('Dp') sym('mg') sym('Ix') sym('Iy') sym('Iz') sym('xg') sym('yg') sym('zg') sym('rg') sym('mm') sym('Imx') sym('Imy') sym('Imz') sym('rm') sym('mw') sym('xw') sym('yw') sym('zw') sym('kw') sym('Iwx') sym('Iwy') sym('Iwz') sym('Dw') sym('g')],[lam kp Dp mg Ix Iy Iz xg yg zg rg mm Imx Imy Imz rm mw xw yw zw kw Iwx Iwy Iwz Dw g]));

sys_def $=$ ss(A_def,B_def,C_def,D_def);

\%\%\%\%\%\%\%\%\%\%\%\%\%\%\%\% No spin SYSTEM \%\%\%\%\%\%\%\%\%\%\%\%\%\%\%\%\%\%\%\%\% A_off = double(subs(A, [sym('lam') sym('kp') sym('Dp') sym('mg') sym('Ix') sym('Iy') sym('Iz') sym('xg') sym('yg') sym('zg') sym('rg') sym('mm') sym('Imx') sym('Imy') sym('Imz') sym('rm') $\operatorname{sym}(' m w ') \operatorname{sym}(' x w ') \operatorname{sym}(' y w ') \operatorname{sym}(' z w ') \operatorname{sym}(' k w ') \operatorname{sym}(' I w x ') \operatorname{sym}(' I w y ') \operatorname{sym}(' I w z ') \operatorname{sym}(' D w ')$ sym('g')],[0 kp Dp mg Ix Iy Iz xg yg zg rg mm Imx Imy Imz rm mw xw yw zw kw Iwx Iwy Iwz Dw g]));

B_off = double(subs(B, [sym('lam') sym('kp') sym('Dp') sym('mg') sym('Ix') sym('Iy') sym('Iz') 
sym('xg') sym('yg') sym('zg') sym('rg') sym('mm') sym('Imx') sym('Imy') sym('Imz') sym('rm') sym('mw') sym('xw') sym('yw') sym('zw') sym('kw') sym('Iwx') sym('Iwy') sym('Iwz') sym('Dw') sym('g')],[0 kp Dp mg Ix Iy Iz xg yg zg rg mm Imx Imy Imz rm mw xw yw zw kw Iwx Iwy Iwz Dw g]));

C_off = double( $\operatorname{subs}(\mathrm{C},[\operatorname{sym}(' \mathrm{lam}$ ') sym('kp') sym('Dp') sym('mg') sym('Ix') sym('Iy') sym('Iz') sym('xg') sym('yg') sym('zg') sym('rg') sym('mm') sym('Imx') sym('Imy') sym('Imz') sym('rm') sym('mw') sym('xw') sym('yw') sym('zw') sym('kw') sym('Iwx') sym('Iwy') sym('Iwz') sym('Dw') sym('g')],[0 kp Dp mg Ix Iy Iz xg yg zg rg mm Imx Imy Imz rm mw xw yw zw kw Iwx Iwy Iwz Dw g]));

D_off = double(subs(D, [sym('lam') sym('kp') sym('Dp') sym('mg') sym('Ix') sym('Iy') sym('Iz') sym('xg') sym('yg') sym('zg') sym('rg') sym('mm') sym('Imx') sym('Imy') sym('Imz') sym('rm') sym('mw') sym('xw') sym('yw') sym('zw') sym('kw') sym('Iwx') sym('Iwy') sym('Iwz') sym('Dw') sym('g')],[0 kp Dp mg Ix Iy Iz xg yg zg rg mm Imx Imy Imz rm mw xw yw zw kw Iwx Iwy Iwz Dw g]));

sys_off =ss(A_off,B_off,C_off,D_off);

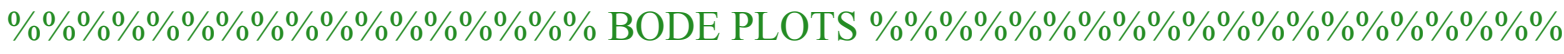

$\%$ In Complete_1DOF must go to linearization and set $\mathrm{G}=[\mathrm{x} 1 ; \mathrm{x} 2]$;

figure(1)

bodeplot(sys_hand, sys_off, sys_def, opts)

legend('Hand', 'Gyro Off', 'Gyro On')

hold on

$\% \% \% \% \% \% \% \% \% \% \% \% \% \% \% \%$ DEFINE TORQUE INPUT $\% \% \% \% \% \% \% \% \% \% \% \% \% \% \% \% \% \%$

$\%$ Here Torque input is $\mathrm{dT}$, the torque that goes into linear system 
Amp $=7.7 \%$ Amplitude of torque input

freq $=8 \%$ Set frequency of the torque input

time_duration $=2 ; \%$ Set duration of torque input

$\mathrm{t} \_$lin $=0: 0.001$ :time_duration; \% This is to make $\mathrm{t}$ evenly spaced for 1 sim

$\operatorname{tspan}=[0$, time_duration $] ; \%$ time over which ODE is solved

$\mathrm{dT}=$ Amp*sin(2*pi*freq*t_lin); \% Sinusoidal input (dT means deviation from equil point)

$\% \mathrm{dT}=0 * \mathrm{t}_{-}$lin $+\mathrm{A} ; \quad \%$ Step Input

$\% \mathrm{dT}=500 *$ heaviside(0.001-t_lin);

\%\%\%\%\%\%\%\%\%\%\%\%\%\% DEFINE Default NL SYSTEM \%\%\%\%\%\%\%\%\%\%\%\%\%\%\%\%\%\%

Tw1_eq = double(subs(Tw_eq, [sym('lam') sym('kp') sym('Dp') sym('mg') sym('Ix') sym('Iy') sym('Iz') sym('xg') sym('yg') sym('zg') sym('rg') sym('mm') sym('Imx') sym('Imy') sym('Imz') sym('rm') sym('mw') sym('xw') sym('yw') sym('zw') sym('kw') sym('Iwx') sym('Iwy') sym('Iwz') sym('Dw') sym('g')],[lam kp Dp mg Ix Iy Iz xg yg zg rg mm Imx Imy Imz rm mw xw yw zw kw Iwx Iwy Iwz Dw g]));

Tp1_eq = double(subs(Tp_eq, [sym('lam') sym('kp') sym('Dp') sym('mg') sym('Ix') sym('Iy') sym('Iz') sym('xg') sym('yg') sym('zg') sym('rg') sym('mm') sym('Imx') sym('Imy') sym('Imz') sym('rm') sym('mw') sym('xw') sym('yw') sym('zw') sym('kw') sym('Iwx') sym('Iwy') sym('Iwz') sym('Dw') sym('g')],[lam kp Dp mg Ix Iy Iz xg yg zg rg mm Imx Imy Imz rm mw xw yw zw kw Iwx Iwy Iwz Dw g]));

$\mathrm{Tw}=\mathrm{dT}+\mathrm{Tw} 1 \_\mathrm{eq} ;$

$\mathrm{Tp}=\mathrm{Tp} 1 \_$eq; 
$\mathrm{X} 0=[0,0,0,0] ; \quad \%$ initial values

[t,X]=ode45(@LINvsNL,tspan,X0,[],t_lin,Ix,Iy,Iz,mg,rg,Imx,Imy,Imz,mm,rm,xg,yg,zg,lam,kp, Dp,Tp,g,Iwx,Iwy,Iwz,mw,xw,yw,zw,Tw,kw,Dw,Rotation,orientation);

x1_NL $=(180 /$ pi) $* X(:, 1) ; \%$ theta (angle of the pitch)

x2_NL $=(180 /$ pi $) * X(:, 2) ; \%$ phi (precession angle $)$

x3_NL $=(180 /$ pi $) * X(:, 3) ; \%$ thetadot

x4_NL = (180/pi)*X(:,4); \%phidot

clear X;

[t_off,X_off]=ode45(@LINvsNL,tspan,X0,[],t_lin,Ix,Iy,Iz,mg,rg,Imx,Imy,Imz,mm,rm,xg,yg,zg, 0,kp,Dp,Tp,g,Iwx,Iwy,Iwz,mw,xw,yw,zw,Tw,kw,Dw,Rotation,orientation);

x1_NL_off $=(180 /$ pi)*X_off(:,1); \% theta (angle of the pitch)

x2_NL_off $=(180 /$ pi $) * X \_o f f(:, 2) ; \%$ phi (precession angle $)$

x3_NL_off $=(180 /$ pi $) * X \_o f f(:, 3) ; \%$ thetadot

x4_NL_off $=(180 /$ pi $) * X \_o f f(:, 4) ; \%$ phidot

clear X_off;

\%\%\%\%\%\%\%\%\%\%\% PLOT LINEAR SYSTEM W GYRO ON \%\%\%\%\%\%\%\%\%\%\%\%\%\%

y_Lin $=1$ sim(sys_def,dT,t_lin,X0);

y1_Lin_on $=(180 /$ pi)*y_Lin(:,1); \% x1 = theta in deg

y2_Lin_on $=(180 /$ pi $) * y \_L i n(:, 2) ; \%$ x2 $=$ phi in deg

clear y_Lin;

figure(2)

plot(t_lin,y1_Lin_on,'b');

$x \lim \left(\left[\begin{array}{ll}0 & 1\end{array}\right]\right)$ 
xlabel('t (s)');

ylabel('Pitch angle (deg)');

title(['Sinusoidal input at ' num2str(freq) ' Hz']);

grid on;

hold on;

$\% \% \% \% \% \% \% \% \% \% \% \% \% \%$ PLOT LINEAR SYSTEM W GYRO Off $\% \% \% \% \% \% \% \% \% \% \% \% \%$

y_Lin_off $=1 \operatorname{sim}($ sys_off,dT,t_lin,X0);

y1_Lin_off $=(180 / p i) * y \_L i n \_o f f(:, 1) ; \% x 1=$ theta in deg

y2_Lin_off $=(180 / p i) * y \_L i n \_o f f(:, 2) ; \% \times 2=p h i$ in deg

clear_y_Lin;

plot(t_lin,y1_Lin_off,'g');

hold on;

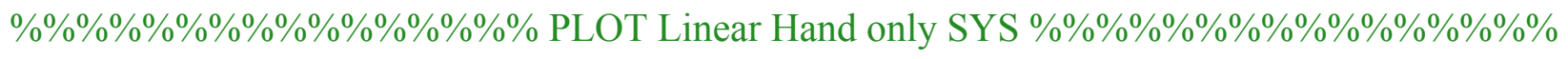

y_hand $=1 \operatorname{sim}($ sys_hand,dT,t_lin,X0);

y1_hand $=(180 / \text { pi })^{*}$ y_hand $(:, 1) ; \%$ x1 $=$ theta

$\mathrm{y} 2$ hand $=(180 / \mathrm{pi}) * \mathrm{y} \_$hand $(:, 2) ; \% \mathrm{x} 2=\mathrm{phi}$

clear y_hand;

plot(t_lin,y__hand,'k');

hold on;

$\% \% \% \% \% \% \% \% \% \% \% \% \% \%$ PLOT NL SYSTEM W Gyro ON \%\%\%\%\%\%\%\%\%\%\%\%\%\%\%\%\%\%

$\operatorname{plot}\left(\mathrm{t}, \mathrm{x} 1 \_\mathrm{NL}, \mathrm{r}_{--}\right)$;

hold on;

$\% \% \% \% \% \% \% \% \% \% \% \% \%$ PLOT NL SYSTEM W Gyro OFF \%\%\%\%\%\%\%\%\%\%\%\%\%\%\%\% 


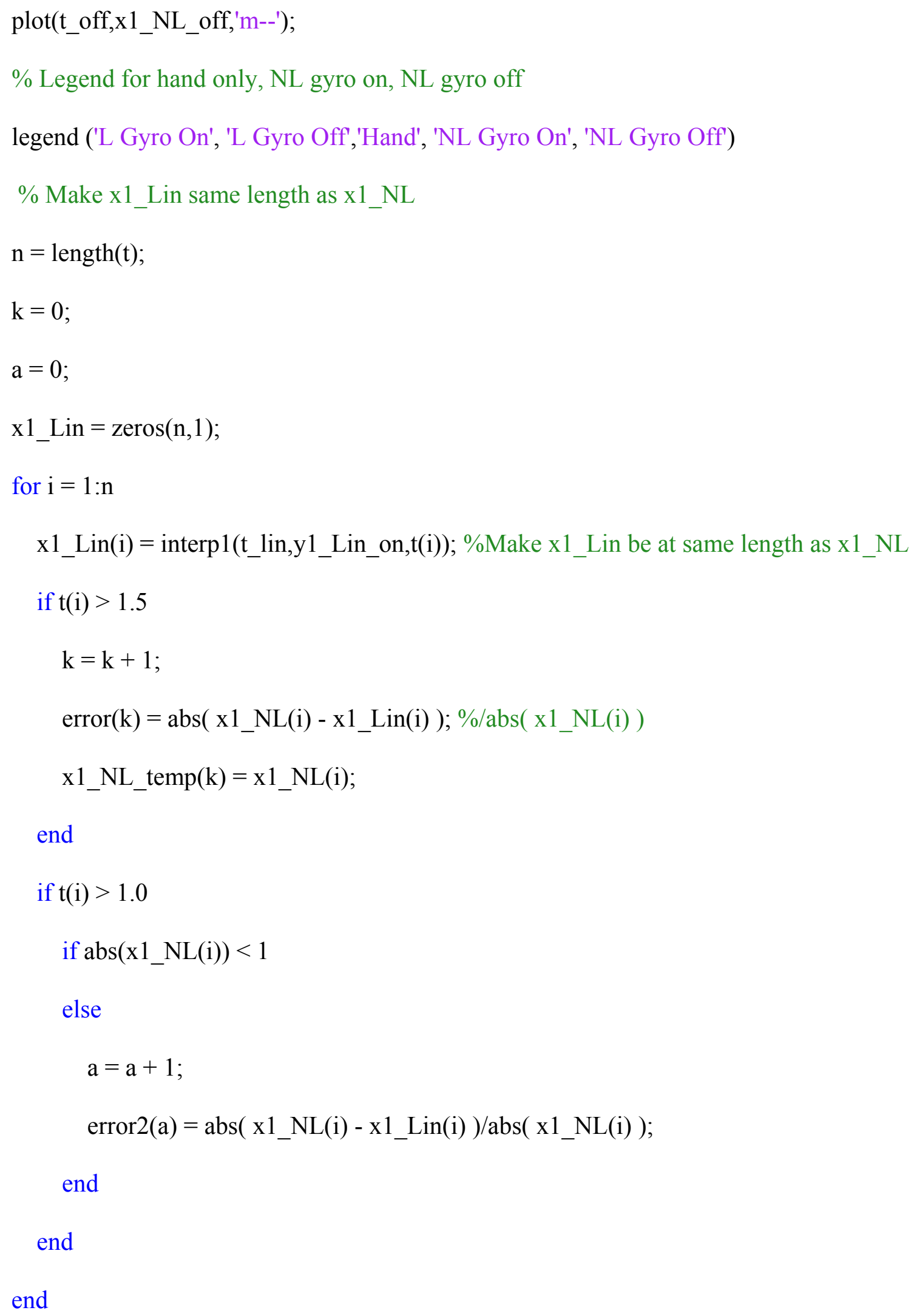


error_avg $=$ mean(error)

Max_displacement $=\max \left(\mathrm{x} 1 \_\right.$NL_temp $)$

Max_Amplitude $=(1 / 2) *\left(\max \left(x 1 \_N L \_t e m p\right)-m i n\left(x 1 \_N L \_t e m p\right)\right)$

figure

plot(error)

title('Error')

figure

plot(x1_NL_temp)

title('NL Plot during SS')

clear error

clear x1_NL_temp

\section{E.3 LINvsNL.m (partial)}

function $[\mathrm{xdot}]=$

LINvsNL(t_curr,x,t_vec,Ix,Iy,Iz,mg,rg,Imx,Imy,Imz,mm,rm,xg,yg,zg,lam,kp,Dp,Tp,g,Iwx,Iwy,I

wz,mw,xw,yw,zw,Tw_vec,kw,Dw,Rotation,orientation)

$\mathrm{xdot}=\operatorname{zeros}(4,1) ; \%$ Initialize $\mathrm{xdot}$ as a column vector because ode 45 expects it

$\%$ Torque input here is $\mathrm{T}=\mathrm{dT}+\mathrm{Teq}$

$\mathrm{Tw}=$ interp1(t_vec',Tw_vec',t_curr); \% Torque at current time step

$x \operatorname{dot}(1)=x(3)$

$x \operatorname{dot}(2)=x(4) ;$

$x \operatorname{dot}(3)=$

$x \operatorname{dot}(4)=$

end 